Hanse-Studien / Hanse Studies Hanse-Wissenschaftskolleg Delmenhorst

Hanse Institute for Advanced Study

\title{
Band 1 / Volume 1
}

Nikola Grahek

\section{Feeling Pain and Being in Pain}

\author{
bis \\ Bibliotheks- und Informationssystem der Universität Oldenburg \\ 2001
}




\title{
Hanse-Studien / Hanse Studies Hanse-Wissenschaftskolleg Delmenhorst Hanse Institute for Advanced Study
}

\author{
herausgegeben von
}

Prof. Dr. Dr. Gerhard Roth und Uwe Opolka

In der Reihe Hanse-Studien / Hanse Studies erscheinen - in deutscher oder englischer Sprache - unveröffentlichte Forschungsarbeiten, die am Hanse-Wissenschaftskolleg (HWK) in Delmenhorst entstanden sind, sowie Berichte über vom HWK durchgeführte Konferenzen. Das Hanse-Wissenschaftskolleg ist eine gemeinnützige Stiftung privaten Rechts der Länder Bremen und Niedersachsen sowie der Stadt Delmenhorst. Es wurde 1995 gegründet und nahm 1997 seine Arbeit auf. Seine Hauptaufgabe besteht in der Stärkung des überregional und international anerkannten Forschungspotentials der umliegenden Universitäten und Forschungseinrichtungen, insbesondere der Universitäten Oldenburg und Bremen. Seine derzeitigen Arbeitsschwerpunkte liegen auf den Gebieten Meeres- und Klimaforschung, Neuro- und Kognitionswissenschaften, Sozialwissenschaften / Sozialpolitik sowie auf interdisziplinären Projekten. In diesen Bereichen beruft es Fellows und führt Tagungen durch.

Anschriften der Herausgeber:

Prof. Dr. Dr. Gerhard Roth Hanse-Wissenschaftskolleg

Lehmkuhlenbusch 4 27753 Delmenhorst Telefon: 042 21/91 60-108 Telefax: 042 21/91 60-199 e-mail: gerhard.roth@h-w-k.de
Uwe Opolka

Hanse-Wissenschaftskolleg

Lehmkuhlenbusch 4

27753 Delmenhorst

Telefon: 042 21/91 60-109

Telefax: 042 21/91 60-199

e-mail: uopolka@h-w-k.de
Verlag/Druck/

Vertrieb

\author{
Bibliotheks- und Informationssystem der \\ Carl von Ossietzky Universität Oldenburg (BIS) - Verlag \\ Postfach 2541, 26015 Oldenburg \\ Telefon: +49-4 41-7 98-22 61 \\ Telefax: +49-4 41-7 98-40 40 \\ e-mail: verlag@uni-oldenburg.de
}

ISBN 3-8142-0780-7 


\section{Contents}

Acknowledgments

1. Introduction 7

2. The Biological Function and Importance of Pain 12

3. Dissociation Phenomena in Human Pain Experience 31

4. Pain Asymbolia 42

5. How Is Pain Without Painfulness Possible? 51

6. Conceptual and Theoretical Implications of Pain Asymbolia $\quad 70$

7. Pain Quality and Painfulness Without Pain 89

8. Not Caring for Pain 105

9. C and All That Fibers 131

References 153

$\begin{array}{ll}\text { Index } & 160\end{array}$ 
For Ana, Ivan and Nela

$$
\text { and }
$$

to the memory of Aleksandar Kron 


\section{Acknowledgments}

This book was conceived and written while I was fellow of the Hanse-Wissenschaftskolleg (Hanse Institute for Advanced Study) in Delmenhorst (Germany). I am grateful to the Rector of Hanse Institute, Gerhard Roth, for support, encouragement and understanding. My special thanks go to my host, Hans Flohr, for his interest, friendship and fruitful discussions that we had. I am indebted to the staff of the Hanse Institute and to all fellows for the wonderful time that I had during my twelve months stay. I am particularly grateful to Mechthild Harders-Opolka and to Uwe Opolka for their friendship and support. For long-term support and lasting interest in my work I am indebted to Daniel Dennett, Boris Velichkovsky, Jay Rosenberg, David Rosenthal, Helmut Hildebrandt, Peter Bieri, Milos Arsenijevic, Zivan Lazovic, Jovan Arandjelovic and Leon Kojen. 


\section{Introduction}

This book is principally devoted to the thorough consideration and general theoretical appreciation of the two most radical dissociation syndromes to be found in human pain experience. The first syndrome is related to the complete dissociation between sensory and affective, cognitive and behavioral components of pain, while the second one has to do with absolute dissociation that goes into opposite direction: the full dissociation of affective components of human pain experience from its sensory-discriminative components. The former syndrome can be called pain without painfulness and the latter one painfulness without pain. In the first case, one is able to feel pain but is not able to be in pain, while in the second case one is able to be in pain but not able to feel pain. Taking into account our common experience of pain, it might well seem to us that the two syndromes just described are inconceivable and, thus, impossible. In order to make them more intelligible and, thus, less inconceivable, the crucial distinction between feeling pain and being in pain is introduced and explained on conceptual and empirical grounds. But the main point is that pain without painfulness as well as painfulness without pain are, however bizarre or outlandish, nonetheless possible, for the simple reason that ample clinical evidence conclusively shows that they can be found in human pain experience. So, the question is not whether they exist or can exist, but what they can teach us about the true nature and structure of human pain experience. Accordingly, the major theoretical aim of this book will be to appreciate what lessons are to be learned from the consideration of these syndromes as far as our very concept or, more importantly, our very experience of pain is concerned.

The first lesson being that pain, although appearing to us as simple, homogenous experience, is actually a complex experience comprising sensory-discriminative, emotional-cognitive and behavioral components which commonly go together, but may well be disconnected and thus exist, to our great astonishment, separately. The second lesson being that pain, once deprived of all its affective, cognitive and behavioral components, loses all its representational and motivational 
force: that it is no longer a signal of threat or damage and doesn't move ones mind and body in any way. The third lesson being that pain, when deprived of its sensory-discriminative components, comes to such sensory indeterminacy that it cannot be distinguished from other unpleasant sensations or sensations of other quality, and looses all informational power with regard to the location, intensity, temporal profile and nature of harmful stimuli. Thus, the two most radical dissociation syndromes in human pain experience may well reveal to us the really complex nature of pain; its major constitutional elements; the proper role that they play in overall pain experience; the way that they work together as well as the basic neural structures and mechanisms that subserve them.

Pain without painfulness is to be found in patients who suffer from the so-called pain asymbolia, and it is characteristic for these patients that they feel pain upon harmful stimulation, but that it doesn't in any way represent for them the sign of threat or danger: that they do not care for it at all and even smile or laugh at it. But as I will try to show, this pain which doesn't represent any threat or danger to the subject, poses gross threat both to the subjectivist and to the objectivist conceptions or interpretations of the true nature and structure of human pain experience. That is, to the subjectivist view that the sensation of pain with its distinctive phenomenal content or quality - the what-itis-likeness of pain - is the essential component of our total pain experience and plays the central or fundamental role in it. And also to the objectivists claim that the feeling of pain is to be understood just as the awareness of objective bodily state of affairs: as the perception or sensory representation of bodily or tissue damage. Actually, the consideration of pain asymbolia syndrome might well help us to clearly see the rights and wrongs of the subjectivist and objectivist conceptions of pain. In other words, it may help us to see what is the proper role of pain sensation or pain quality in overall pain experience as well as to understand the proper mode in which pain represents physical damage to the body part or at least threat to the physical well-being.

Pain asymbolia syndrome or pain without any painfulness is yet in another conceptual and theoretical respect important for the proper 
understanding of human pain experience. Namely, this syndrome holds a unique position among reactive dissociation syndromes to be found in human pain experience. As I will try to show, it is to be regarded as the only clear-cut case in which quite severe pain is not at all experienced as unpleasant and in which there are no traces of any other aversive attitude toward it. Besides, it is the only case of complete, thoroughgoing indifference to pain, and is the sole one that is distinguished by total absence of any appropriate pain behavior and any tendency or disposition toward such behavior. But due to its purity and simplicity, the case of pain asymbolia or of pain without painfulness is also the most perspicuous case of the pain that has lost all its biological function and significance: it is recognized by the subject as pain and is felt as located at certain body part, but means nothing to him and is at best something that one laughs or smiles at. Bentham has said that Nature has placed mankind "under the governance of two sovereign masters: pain and pleasure" (1948, p. 1). But which pain? What part of pain? How pain has power over mankind?

To get an answer to these questions, we have to look more closely at the biological significance and function of pain, and the opening chapter of this book will exactly be devoted to the consideration of these issues. The analysis of the biological significance and function of pain, as well as the inquiry into the true nature and structure of pain through the study of dissociation phenomena in human pain experience, will always be grounded on the consideration of the basic neural structures and mechanisms that subserve and are responsible for the appearance of pain experience. But at one point the "hard" problems of consciousness, related to the very intelligibility of the connection between the experience of pain, as eminently subjective or phenomenal state, and the activity of the corresponding neural structures and mechanisms, will come into play: why their activity gives rise to the sensation of pain and not to some other sensation? why it gives rise to any sensations at all? The closing chapter of the book will be dedicated to the consideration of these intelligibility issues, and I will claim that the phenomenal or subjective state of pain and the neural structures responsible for its appearance share one common 
property that makes their connection intelligible. Namely, once this common property is realized, we will only have to apply the simple hermeneutical instruction: just connect. However, my theoretical ambition will not be to solve or resolve the "hard" problems of consciousness related to the experience of pain, but rather to lift some of the burden that hangs around them.

Before I go into all these considerations, I have to make one more remark related to the major theoretical intentions of this book. Namely, throughout the book, from the very beginning to the very end, I am doing my best to resist the strong philosophical temptation to judge in advance or according to our preconceived ideas whether pain without painfulness and painfulness without pain should or should not be treated as cases of pain at all. It is more fascinating and important to learn from these bizarre and puzzling cases something about the true nature and structure of human pain experience, than to enjoy in the fact that another piece of evidence speaks in favor of one's cherished theory or to despair if it doesn't. In other words, in this book the reader will not find another philosophical theory of pain that is supposed to conclusively support or absolutely discount the deeper metaphysical, semantic and epistemological intuitions that lie behind the subjectivist or objectivist positions in the philosophy of mind. The main theoretical or philosophical ambition of the book is the more modest one: to appreciate the rights and wrongs of these intuitions so as to show that a less doctrinaire and more balanced approach to the study of mind/brain phenomena is quite salutary and, indeed, highly recommendable. At this stage of pain research and theory, we still do not have a fully satisfactory or fully adequate conceptual and neural model of pain that would explain all puzzling phenomena to be found in human pain experience and put pain under firm control. However, we do know much more about pain then we knew only few decades ago, and we do have a much better and broader insight into the working of its major biological and psychological mechanisms that are of vital importance for the survival and protection of human kind and, not to forget, animal species. If this book is to be appreciated by its readers as the modest contribution to 
such insight, it will definitely serve - to author's great satisfaction its major purpose. 


\section{The Biological Function and Importance of Pain}

The capacity to feel pain upon external harmful stimulation or upon internal bodily damages is certainly the most precious gift bestowed on us by Mother Nature for self-protective purposes. However, those who are lucky enough to be able to feel pain when their bodies are exposed to damaging or potentially damaging stimuli, have highly ambiguous or even paradoxical attitude toward that gift. And this attitude is best captured by the title of the book which rightly says of pain that it is The Gift Nobody Wants. For it is true that, when in pain, particularly an intrusive and severe one, we dislike it very much, and will do anything to get rid of it or at least to alleviate it. However, when not in pain, we certainly welcome having that highly precious gift landed to us by Mother Nature, for a moment's reflection will convince us what would happen to the integrity of our body and mind if we were from birth or by disease deprived of it. The most dramatic and frightening evidence comes from the consideration of people who suffer from the so-called congenital analgesia, and the first case is said to have been reported by Dearborn (1932). His patient made a living with a human pincushion act, and crucifixion had to be called off when a woman in the audience fainted after a spike was driven through one hand. But the best-documented of all cases of congenital analgesia is the case of Miss C., a young Canadian girl who was a student at the McGill University in Montreal:

As a child, she had bitten off the tip of her tongue while chewing food, and has suffered third-degree burns after kneeling on a hot radiator to look out of the window. When examined ... she reported that she did not feel pain when noxious stimuli were presented. She felt no pain when parts of her body were subjected to strong electric shock, to hot water at temperatures that usually produce reports of burning pain, or to prolonged icebath. ... A variety of other stimuli, such as inserting a stick up through the nostrils, pinching tendons, or injections of histamine under the skin - which are normally considered as form of torture - also failed to produce pain. 
Miss C. had severe medical problems. She exhibited pathological changes in her knees, hip and spine, and underwent several orthopedic operations. Her surgeon attributed these changes to the lack of protection to joints usually given by pain sensation. She apparently failed to shift her weight when standing, to turn over in her sleep, or to avoid certain postures, which normally prevent the inflammation of joints.

All of us quite frequently stumble, fall or wrench a muscle during ordinary activity. After these trivial injuries, we limp a little or we protect the joint so that it remains unstressed during the recovery process. This resting of the damaged area is an essential part of its recovery. But those who feel no pain go on using the joint, adding insult to injury. (Melzack and Wall, 1988, pp. 4-5)

This stunning behavior, with disastrous consequences, is something that Paul Brand has frequently observed among his leprosy patients in the land called "the land of painlessness". Namely, leprosy bacilli destroy the peripheral nociceptors which respond to damaging or potentially damaging stimuli and evoke pain sensation. As these bacilli favor cooler parts of the body, they usually do not affect warm regions such as the armpit, but leave the extremities - particularly hands and foots - completely unprotected from injuries for which patients do not care or bother at all. As Paul Brand has noticed, half of the problem with leprosy patients and their injuries is painlessness. Absolute carelessness in leprosy patients toward injuries, and complete absence of any attempts to protect from further insults the already damaged areas of their bodies, Paul Brand has in most dramatic way experienced with one young boy who was working in the weaving shop of the leprosy sanatorium. The young weaver was working vigorously at a loom, probably showing off for the director and his guest, when Brand spotted the traces of blood on the cotton cloth:

"May I see your hand," I yelled to the weaver ... He held out a deformed, twisted hand with shortened fingers. The index finger had lost maybe a third of an inch in length, and as I looked 
closer I saw naked bone protruding from a nasty, septic wound. This boy was working with a finger cut to the bone!

"How did you cut yourself?" I asked. He gave a nonchalant reply: "Oh, it's nothing. I had a pimple on my finger, and earlier it bled a little. I guess it's opened up again." (Brand and Yancey, 1997, p. 89)

Deprived of the ability to feel any pain on account of noxious or potentially noxious stimuli, Miss C. and Brand's leprosy patients were deprived, with disastrous consequences, of the most sophisticated and most efficient self-protective psycho-physical system to be found in nature: the system which serves as a reliable alarm mechanism which warns organisms about damaging or potentially damaging incidents in the environment, monitors the extent of the damages inflicted, and induces them to take appropriate actions or to refrain from performing actions that would add insult to already damaged body parts. On its most elementary level, this system is actually built-up of two subsystems: (1) the avoidance system and, (2) the restorative or repair system. In more picturesque terms, the first one can be called the "external pathologist", while the second one can be labeled as the "internal pathologist". Although both sub-systems function as alarm or warning systems, they markedly differ in their functions, targets, the neural structures and mechanisms that subserve them as well as in the characteristic behavior that they induce. In order to survive and preserve intact their vital physical and mental capacities, organisms have to protect their bodies from threatening or destructive stimuli coming from the environment or from their own actions; besides, they have to protect from further insults the already damaged areas of their bodies. The first two protective functions are carried by the avoidance system, while the third one (until recently, largely neglected in pain research and evolutionary thinking) is performed by the restorative or repair system.

The avoidance system is sensitive to and reacts to mechanical, thermal and chemical noxious or potentially noxious stimuli. Stimuli of the latter kind are to be understood as stimuli which, if prolonged, would damage the tissue. The system that can reliably detect and im- 
mediately react, by withdrawal or removal, to such stimuli, has a powerful and sophisticated preventive capacity; and this capacity has great protective and survival value. For instance, the system would have to respond to temperatures around $48^{\circ} \mathrm{C}$ at which nerve substance begins to suffer injury; or, it would have to respond to the pressure of the torn or needle just below the pressure sufficient for the thorn or instrument to break into the skin. As far as the neural basis of the avoidance system is concerned, it has been experimentally established - by the use of the technique if intraneural micro-recording and micro-stimulation - that in humans and in various animal species exist neural structures that preferentially respond to noxious or potentially noxious stimuli. Such physiologically specialized neural structures were found among small, high-threshold A delta myelinated, fast conductive fibers (conductive velocity 6 to $30 \mathrm{~m} / \mathrm{sec}$ ), and small $\mathrm{C}$ unmyelinated, polymodal, slow conductive (conductive velocity 0.5 to $1.5 \mathrm{~m} / \mathrm{sec}$ ) fibers (Price and Dubner, 1977; Wall and McMahon, 1985; Torebjörk, Vallbo and Ochoa, 1987; Ochoa and Torebjörk, 1989). This means that it can take more than a second for the nerve impulses conducted by $\mathrm{C}$ fibers to reach the spinal cord from the stimulated foot, while the nerve impulses transmitted by A delta fibers from the same part of the body have reached it "long before". Qualitatively speaking, the excitation of A delta nociceptive fibers corresponds to the fast, sharp, pricking pain (first pain or alarm pain), while the activity of $\mathrm{C}$ nociceptive fibers is related to slaw, dull or burning pain (second pain, or to speculate a bit, remembrance pain). Both kinds of pain, on cutaneous level, have accurate localization, and such function appears to be reserved for pain "as a signal of noxious challenge to the body surface which needs to be well localized for the purposes of removal of agent, projection of the injured site or inhibition by gentle rubbing or scratching" (Ochoa and Torebjörk, 1989, p. 593). This is the main reason why the avoidance system can be called the "external pathologist". The activity both of A delta nociceptive and $\mathrm{C}$ nociceptive fibers is closely and strongly connected to withdrawal reflexes such as flexion reflex and corneal reflex. From the more general behavioral point of view, their activity is followed by positive bodily reactions: movement and manipulation. From the 
biological point of view, the avoidance system is to be considered as basically surface, skin protective system: the part of the body that is most frequently exposed to noxious or potentially noxious stimuli. Finally, from the clinical point of view or the assessment of inflicted injury, the avoidance system is related to transient pain - no damage or light damage to the body tissue - as well as to the early phases of acute pain or frank damage to the body tissue:

The alerting function of acute pain reflects the phasic activation of sensors (nociceptors) by potentially dangerous stimuli exceeding the physiological range. This warning purpose of pain is most evident as concerns skin, which is exposed to external dangers. Acute, cutaneous pain evokes, thus, motor withdrawal and/or 'flight' reaction, protective responses intended to discontinue exposure to the noxious stimulus and, thereby, to terminate the pain. (Millan, 1999, p. 7)

Through the simple psychophysical self-experiment you may yourself quite easily test the qualitative, temporal and spatial differences between the painful sensations evoked by the activity of "your" A delta and $\mathrm{C}$ nociceptive fibers and appreciate how finely these neurons are tuned to detect the potentially dangerous stimuli which would, if not discontinued, frankly damage your body tissue. The first thing that you should do is to hold you fingertip, of moderately normal sensitivity and temperature, for two seconds quite closely to the hot electric light bulb; then you have to press it against the light bulb, keep it there for a second, and remove it quickly - which you will do anyway because the sensation felt as the perception end-point will be very painful. During the first two seconds you are going to feel a diffuse heat (non-painful) sensation that is not related to the activity of your nociceptors, but rather stems from the stimulation of A beta thermoreceptors. Then, near the end of the third second or just before the spontaneous removal of your fingertip from the light bulb, you will suddenly experience the short-lived, stinging or pricking pain felt at the punctate area, and evoked by the activity of your A delta heat nociceptors because the stimulus has become potentially noxious. This sensation will be followed, after a brief interval, by a longer lasting sensation of intense heat and burning pain whose appearance 
is due to the activity of $\mathrm{C}$ polymodal nociceptors. Your A delta heat nociceptors started to respond and evoke withdrawal and stinging pain when the contact temperature came around $48^{\circ} \mathrm{C}$; and that is, as we have already said, exactly the temperature at which nerve substance begins to suffer injury. The message conveyed by the stinging pain elicited via A delta heat nociceptive fibers was that the thermal stimulus has become potentially dangerous. If you had not removed your fingertip instantly from the hot light bulb, it would have been burned. This has happened to me on one occasion when I have, quite unwisely, and out of sheer theoretical curiosity, left my fingertip stay too long on the hot light bulb. I got burned and the blister developed staying on my sore finger for almost a month. That would not have happened if I had taken seriously into account the simple message of "my" A delta heat nociceptive fibers sent to me via stinging pain: that the thermal stimulus is potentially dangerous; that it is a threat for the integrity of my body and my mind. But this is not the only role that pain has played in the whole hurtful story related to my initial foolishness. Namely, once the damage has been done - the long-lasting blister developed - the restorative or repair pain system had fortunately come into play.

This system is sensitive and reacts, by inhibiting movement and manipulation, to lasting changes produced by damages of the joints, muscles and nerve substance, and this is the rationale for labeling it as "internal pathologist". Thus, it prevents the organism to inflict further insults to the already damaged areas of the body. From the neurophysiological point of view, it is subserved by "silent" $\mathrm{C}$ nociceptive chemo fibers that do not respond to noxious stimuli, not even to excessive one, but start to react to chemicals released after the injury has been inflicted (McMahon and Koltzenburg, 1990, pp. 254-255). The system is slow - the transportation of neurotransmitters goes $224 \mathrm{~mm}$ per 24 hours - but is of lasting and steady influence; its activity is usually followed by tenderness (primary and secondary hyperalgesia and hyperesthesia) which spreads around the damaged part of the joint, muscle or nerve, and makes them sensitive to even completely innocuous stimuli. From the biological point of view, the state produced by this system "has the function of greatly diminishing move- 
ment or manipulation. Even though it may be annoying, it is the optimal condition to speed recovery from deep injury" (Melzack and Wall, 1988, p. 107). Consider the behavior of infants and animals while suffering deep injuries and going through the process of healing, and compare it to the behavior of patients congenitally insensitive to pain or of leprosy patients. From the more general behavioral point of view, the action of the restorative system (repair and recover) is followed by negative bodily reactions: refrain from movement and manipulation. In other words, the system is constantly on alert and reliably and efficiently alarms an organism of impermissible actions during the process of healing.

Now that we have a full insight into the major ways in which pain performs its basic protective functions, we should certainly more appreciate and respect that most precious gift of which Miss C., and Brand's leprosy patients were unfortunately deprived. However, people may not only be deprived, genetically or by disease, of that precious gift called the ability to feel pain; they may also become overwhelmed by it to such a degree that the whole mechanism of pain becomes maladaptive and, instead of signaling threat or danger, starts to threaten or endanger, even terrorize, the unfortunate patients. This can happen when, due to primary lesions or dysfunctions in the nervous system, pain becomes chronic, often intractable, serves not anymore as warning system or the symptom of the disease, but is a pain syndrome, and has to be treated as a medical entity of its own. This neuropathic pain (peripheral and central), often followed by hyperpathia (allodynia, hyperesthesia, hyperalgesia), is more a curse or menace than poena: the biological punishment for the behavior that may lead to injury or that may worsen the already inflicted injury; and as every reasonable punishment reminding one or making one learn that some objects or situations should be avoided and some actions strongly resisted. The psychological and behavioral profile of patients suffering from chronic, often intractable, pain is best described by Mitchell in the following way:

Perhaps few persons who are not physicians can realize the influence which long-continued and unendurable pain may have on both body and mind. ... Under such torments the temper 
changes, the most amiable grow irritable, the bravest soldier becomes coward, and the strongest man is scarcely less nervous than the most hysterical girl. Nothing can better illustrate the extent to which these statement may be true than the case of burning pain, or, as I prefer to term it, Causalgia, the most terrible of all tortures which a nerve wound may inflict. (Mitchell, 1872)

And it is true that one of the most dreadful and terrifying examples of the pain system going astray is the case of burning pain or causalgia:

Causalgia typically appears after a high velocity wound (a bullet, shrapnel or knife injury) has damaged a major nerve in a limb. Most patients experience surface pain of a burning quality immediately in the periphery of the injured extremity, and they develop shiny skin and edema in the affected area. The pain worsens and evolves into constant hyperesthesia and allodynia (everything touching the area causes pain). With time, the pain spreads and eventually involves the whole limb. Temperature changes, light touch, friction from clothing, blowing air, movement of the limb, and any stimulus that affects the patient's emotional state can exacerbate the pain. Minor events like a cry of a child, the rattling of a newspaper, or watching a television program can provoke intense pain. Consequently, patients suffer greatly, becoming reclusive, withdrawn, and tragically incapacitated by the pain. (Chapman, Nakamura and Flores, 1999, p. 28)

One of the most amazing things related to patients suffering from causalgia is the fact that severe, intolerable, burning pain, which spreads all over the affected limb, is evoked and strengthened by completely innocuous and highly non-standard stimuli. Imagine excruciating pain being produced by the touch of feather, air puffs, and the scenes on the screen of your television set. It seems that the gift of pain is in such circumstances deprived of any biological meaning for it no longer serves any obvious biological purpose. It is just, as Mitchell has observed, a terrible torture for those who are unfortunate enough to suffer from it. But there are other astonishing examples 
where pain becomes highly inadequate, hyper-protective system, and only arises under completely non-standard stimulus conditions. I am inclined, for reasons that will later become more clear, to call this syndrome, the syndrome of threat hypersymbolia. This syndrome was diagnosed and studied by Hoogenraad and found out in the patient who has suffered extensive lesion in the right parietal cortex:

The patient was transferred to an institution for rehabilitation; he was reassessed after eight months. With eyes closed he had loss of superficial sensation (pain and touch) in the left side of his body, more severely in the arm than in the leg, trunk, and face, the distal parts of the extremities being affected most. No delayed pain reaction occurred. ... Vibration was not perceived. There was lack of awareness of the left half of his body and inability to move his left hand and fingers without visual control. With his eyes open and his gaze directed at his left hand, the patient was able to open and close the hand very slowly. There were no sensory abnormalities on the right side of his body. On seeing that the left part of his body was approached for sensory testing, the patient invariably made a brisk withdrawal movement; at the same time he felt a burning pain that was accompanied by grimacing (italics mine). On moving about, an incidental contact that was not anticipated did not result in pain and withdrawal. When the patient himself approached his left arm with his right hand there was neither pain nor withdrawal. (Hoogenraad et al., 1994, p. 851)

The peculiarity or the mystery of the pain that this patient felt and of the brisk withdrawal reactions that he displayed, are certainly to be found in the fact that, both the withdrawal of the arm as if it had been stung and the burning pain felt in it which was accompanied with grimacing, were exclusively evoked by visual stimuli: solely when he saw the arm being approached by someone. As the authors of the study have remarked, a major difference from classical anesthesia dolorosa - pain in an area or region which is anesthetic - "was that the painful reactions in the patient did not occur spontaneously" (Hoogenraad et al., 1994, p. 851). How are we then supposed to understand these highly bizarre pain reactions? Well, for the patient's 
initial withdrawal reaction when the examiner's instrument was seen to approach his left arm, we could find some explanation or understanding in our own experience or reactions on such occasions. Namely, it often happens that during sensory testing or injection administration we initially withdraw the arm briskly upon seeing the examiner's needle or the nurses' syringe approaching it. It also often happens that we are actually reproached by the examiner or the nurse for reacting improperly to their approaches: "I have not even touched you, let alone hurt you!" But we know the trick; we are well aware that it will hurt, once we are really pricked by the needle or syringe. The sight of the needle or of the syringe approaching the arm was rightly assessed as the threatening visual stimuli, and the arm was consequently spontaneously withdrawn from the threatening or potentially dangerous object. Of course, on the next trial we usually put some effort to control or suppress the avoidance behavior for our own benefit. Moreover, and more to the point, the repeated approaches of the needle or syringe that were really not followed by the expected touch and hurt, would convince us that they do not anymore represent the threat or danger to the integrity of our body, so that the next visual presentation of these objects in the vicinity of the arm would not evoke avoidance behavior. As you are soon to learn, under these repeated unfulfilled expectations, "our" specialized threat detection neurons situated in the sensory association area of the posterior parietal cortex would actually become "convinced" that the visually presented objects do not anymore present threat or danger and, thus, need not be avoided; and they would come to that "conviction" or "assurance" by dramatically decreasing their mean discharge frequencies in response to such visual stimuli to the final point of complete unresponsiveness. But patient's neurons specialized for the detection of novel or threatening visual stimuli obviously never came nor did have a chance to ever come to that "conviction" or "assurance"; they were not able to learn to discriminate between real and seeming threat, so that the patient, during sensory testing, invariably made a brisk withdrawal movement whenever his left arm was approached by examiner's instrument. 
His arm ... gave him a lot of trouble ... (for) when he saw the arm being approached by someone it would suddenly move sideways as if it had been stung. The involuntary withdrawal movements of his left arm were so embarrassing that he tied it to his belt. (Hoogenraad et al., 1994, p. 851)

The only exceptions to these embarrassing, uncontrollable, withdrawal movements were to be encountered when the patient himself would approach his left arm with his right hand: there would then be neither pain nor withdrawal. The absence of withdrawal on such occasions is something that we can understand, because we know from our own experience that the withdrawal movement on visual threat will be cancelled if we suddenly approach our left arm with our own right hand, although the avoidance reaction would have been evoked if somebody else had suddenly approached it with his hand. But the invariable, persistent withdrawal of the arm, whenever it was approached by someone else, still remains a mystery. Even more mysterious is the regular appearance of burning pain evoked solely by visual stimuli. To make these highly bizarre phenomena less mysterious, we have to take into account and consider in more detail the evidence that tells us that there exist, in the sensory association area of the posterior parietal cortex, specialized multi-sensory neurons whose role is to integrate somatosensory nociceptive inputs with visual inputs in order to provide "an overall sense of intrusion and threat to the physical body and self” (Price, 2000, p. 1771).

This evidence comes from the relevant electrophysiological, behavioral and lesion studies in monkeys and humans, and the first thing to be discovered was the existence of neurons, in the area $7 \mathrm{~b}$ of monkey's brain, that were sensitive or responded preferentially to noxious or potentially noxious stimuli. Among these nociceptive neurons, the sub-population of neurons was found that reliably encoded harmful thermal stimuli as well as the degree of their harmfulness, for they started to respond to thermal stimulus intensities around $47^{\circ} \mathrm{C}$ and monotonically increased their mean discharge rates to temperature shifts from $47^{\circ}$ to $51^{\circ} \mathrm{C}$ : that is, the temperature intensities at which nerve substance starts to be damaged or would be damaged if the stimulus was not discontinued (Dong et al., 1994). Complementary 
behavioral studies in monkeys have shown that the stimulus intensityresponse functions of these nociceptive cells closely approximate the stimulus intensity-escape frequency function. In other words, thermal stimulus intensities that evoked over 50 percent of escape responses in monkeys were exactly those to which thermal nociceptive neurons best or most reliably responded (Dong et al., 1994). This makes them perfectly and distinctively suitable to play the role of neurons that alarm an organism to the presence of threatening or potentially dangerous thermal stimuli and evoke avoidance or escape responses. But it has turned out that these neurons do not respond only to somatosensory stimuli, but also to visual stimuli and are in that sense multi-sensory neurons. The visual stimuli to which they best responded were the approach of novel or threatening objects and their mean discharge rate was decreased by iterative presentation of the same novel or threatening object; however, a response could be elicited by showing the object if it was again used to apply a painful stimulus (Robinson and Burton, 1980). So it seems that these multi-sensory neurons are capable of learning and relearning the threatening significance of visual stimuli; of giving assurances and reassurances with regard to the potentially dangerous character of such stimuli. This reminds one of the famous Wittgenstein's remark related to the situations in which we can disclose the true nature and causes of certainty:

The character of the belief in the uniformity of nature can perhaps be seen most clearly in the case in which we fear what we expect. Nothing could induce me to put my hand into a flame, although after all it is only in the past that I have burnt myself. (1968, § 472)

I shall get burned if I put my hand in the fire: that is certainty.

That is to say: here we see the meaning of certainty. (What it amounts to, not just the meaning of the word "certainty".). (1968, § 474)

Namely, one could say that, in the situation when we are watching our hand closely approaching the fire, the certainty that we shall get burned if we put our hand in the fire, is the certainty bestowed upon us by the "certainty" that "our" multi-sensory nociceptive neurons 
have reached by firing vigorously at the visual presentation of the hand approaching closely the fire. When the possibility of giving neurological explanation of one our basic epistemic categories - that of certainty - is at stake, this is the only way, as far as I can see, in which one can give sense and substance to the venerable project of naturalizing epistemology. This project is well under way in cognitive neurosciences where it goes under the more modest and more precise heading: "What can the brain tell us about the mind?" In this context and related to the topic of the neurological grounds of basic forms of certainty, one should also mention that quite recently pertinent electrophysiological studies in awake humans have disclosed that, in the anterior cingulate cortex, exist neurons that respond selectively to painful mechanical and thermal stimuli, and that some of these neurons also responded, like the ones disclosed in monkeys, to threatening or potentially dangerous visual stimuli:

Interestingly, this cell also responded when the patient watched pinpricks being applied to the examiner's fingers. When pinpricks were again applied to the patient, the response started before skin was contacted, suggesting a response to pain anticipation. (Hutchison et al., 1999, p. 404)

Let us, after this necessary digression, go back to the consideration of one more important property of nociceptive multi-sensory neurons. Namely, one should take also into account the significant fact that they will respond or, upon repetitive presentations, stop to respond to visual stimuli, only if these stimuli are located near the arm or face. That is, their receptive fields are not retinotopically but somatotopically organized, and are congruent with cutaneous receptive fields of nociceptive neurons on the arm. This is the reason why they best or only respond to visual stimuli approaching the seen hand at the distance of 10 to $20 \mathrm{~cm}$ or directed at that distance to the face. As a consequence of the visuo-nociceptive integration, the activation of the multi-sensory neurons by a visual stimulus closely approaching the left arm will also activate the corresponding somaesthetic representation of that arm and enhance the activity of thermal nociceptive neurons. If that is the case, it doesn't seem improbable that seeing could produce feeling. 
Lesion and clinical studies have shown that damage of the posterior parietal cortex often results in the motor loss affecting the contralesional upper limb and complete somatosensory loss in that limb: that is, patients are unable to move their arm and are unable to feel, upon stimulation, any sensation in any sensory modality. Neurological somatosensory assessment is usually carried out with the patient's eyes closed. However, in one study conducted on the large sample of patients suffering from somatosensory loss in the contralesional arm, the somatosensory assessment was not carried out only with the patient's eyes closed, but they were also encouraged to look at their affected arm when it was touched by the experimenter. No patient could feel any sensations with the eyes closed, but two patients reported having felt a tactile sensation only if they were allowed to see the arm being touched by the experimenter (Halligan et al., 1997). In one patient, simply the vision on the screen of the affected hand been touched produced reports of tactile sensation in the affected hand, even if no real touch occurred.

Now, the patient that we are interested in could also not feel any sensations (pain and touch) in his left arm when he kept his eyes closed during the sensory testing nor could he move his left hand and fingers during motor testing. However, with his eyes open and his gaze directed at his left hand, he was able to open and close the hand very slowly. It is true that vision was of great help for enabling and facilitating the desperately needed motor performances of the left hand. But vision was also the source of his great embarrassment and undeserved suffering as far as his left arm was concerned: simply the vision of his left arm being approached by examiner's instrument evoked involuntary withdrawal movements and the feeling of burning pain accompanied by grimacing. So here we have another case of seeing producing feeling in the otherwise anesthetic arm, although in this case seeing has evoked the more spectacular feeling of burning pain. But the case that we are interested in should not be treated just as another example of seeing producing feeling, for it is much more than that. It is actually the case in which the human pain protective system has irreparably gone astray causing permanent misery to the unfortunate sufferer. The deeper neural basis of this irreparable dam- 
age to that precious system is to be sought in the neuronal vicious circle that has been established due to the impossibility of the neuronal dissociation or uncoupling that would lead to neuronal relearning. This neuronal vicious circle has put the spell of threat on the unfortunate patient, for he was not anymore able to discriminate between visual stimuli that are really threatening and those that are not: every visual stimulus approaching his hand would, under any conditions, be assessed by him as the potentially dangerous stimulus, and that is why we can speak in his case of the threat hypersymbolia.

But how was the neuronal vicious circle responsible for this highly inadequate and bizarre behavior established? Well, due to the damage of the right posterior parietal lobe, nociceptive neurons situated in sensory association area could not receive anymore somatosensory inputs evoked by noxious mechanical or thermal stimulation of the left arm. However, these neurons are multi-sensory and their responses could be evoked by visual stimuli, although only of specific character. As we have shown, these nociceptive multi-sensory neurons respond best to novel or threatening visual objects and these objects have to be visually presented as closely approaching the arm. When such stimulus is visually presented - for instance, examiner's instrument approaching the left arm - multi-sensory nociceptive neurons will respond and their activation, due to integration, will activate the corresponding somaesthetic representation of the arm; this will in its turn enhance the thermal nociceptive neurons to project the sensation of the burning pain to the left arm and induce brisk removal of the arm from the approaching object. As each new visual presentation of the object, during the sensory testing with eyes opened and the gaze directed at the left arm, gives rise to burning pain in the arm, there is no way in which the threatening significance can be detached or uncoupled from the visual presentation of the instrument approaching the patient's left arm. To put this point into neural terms, there is no way in which patient's multi-sensory neurons responding to visual stimuli can be "reassured" and decrease their mean discharge rate upon the next visual presentation of the instrument or stop to respond to it. In this way the vicious circle is established: the visual stimulus giving rise to burning pain, and the regular appearance of 
this pain upon visual stimulation reinforcing the threatening meaning of the visual stimulus, so that it always recruits the multi-sensory neurons that distinctively respond to visual objects closely approaching the subject's hand.

How vicious that circle really is, may best be appreciated if we start to think how we could help the patient to get out of it. The first strategy that comes to mind is of course the strategy of relearning or retraining the neural mechanism that has gone astray and is responsible for patient's uncontrollable overreactions to visual stimuli. One way to do it would be to repeatedly present the visual object in such a way that its presentation would not be followed by the feeling of the burning pain in the arm. Thus, the multi-sensory neurons situated in the sensory association area of the right posterior parietal lobe would not anymore respond to the relevant visual stimulus and the corresponding feeling of burning pain would not be anymore evoked by their activity. In short, seeing would not anymore produce feeling in the otherwise anesthetic left arm. But this strategy is doomed to failure because, in this patient, every visual presentation of the instrument approaching his left arm invariably produces the feeling of burning pain in that arm. We could also make an attempt to hold his arm firmly while he is watching the instrument approaching his left arm, and try to induce the innocuous tactile sensation by the instrument, hoping that the corresponding visual information may "boost sub-threshold tactile stimulation into conscious awareness" (Halligan et al., 1997, p. 203). This neural retraining strategy would basically consist in the attempt to couple the innocuous character of the eventually evoked tactile sensation to the visual appearance of the instrument. The repetition of this sequence could lead to the assessment of the visually presented object as the mock threat: that is, to the "reassurance" of the multisensory neurons with regard to the threatening significance of the visual stimulus and to the eventual decrease or termination of their responsiveness to such stimuli. The problem with this strategy, which is so salutary and effective in ordinary cases, is that it just wouldn't work in the case of the patient that we are interested in. Namely, in his case each visual presentation of the approaching instrument would cause the feeling of burning pain; and this feeling would inhibit the 
appearance of any other concurrent sensations, particularly innocuous one. As Sherrington has long ago observed, pain can suppress or override all other concurrent sensations, so that tactual sensations evoked by innocuous stimulation could not be felt and associated for their innocuous character to the antecedent or concurrent visual stimulus, in order to rob that stimulus of its threatening meaning when it is again presented.

It would seem a general rule that reflexes arising in species of receptors which considered as sense-organs provoke strongly affective sensations ceteris paribus prevail over reflexes of other species when in competition with them for the use of the "final common path". Such reflexes override and set aside with peculiar facility reflexes belonging to touch organs, muscular sense-organs, etc. As the sensations evoked by these arcs, e.g. "pains", exclude and dominate concurrent sensations, so do the reflexes of these arcs prevail in the competition for possession of the common paths. They seem capable of pre-eminent intensity of action. (Sherrington, 1948, p. 232)

What holds for dominant reflexes is obviously also valid for dominant sensations: for "pains" are certainly capable of pre-eminent intensity of feeling and, consequently, of action. This is another reason why there is no, via the neuronal relearning or retraining, a way out of the vicious circle into which the patient's pain protective mechanism has fallen. This, now vicious self-protective system, is completely encapsulated or totally impenetrable for any functional reorganization. It works under its own, quite justified premises, and makes sense when not considered, as Descartes has observed, from the point of view that is extrinsic to the mechanisms to which it is applied and is a kind of external denomination with regard to them:

A clock composed of wheels and counter-weights no less exactly observes the laws of nature when it is badly made, and does not show the time properly, then when it entirely satisfies the wishes of its maker. ... And although considering the use to which the clock has been destined by its maker, I may say that it deflects from the order of its nature when it does not indicate 
the hours correctly ..., nevertheless I recognize at the same time that this last mode of explaining nature is very different from the other. For this is but a purely verbal characterization depending entirely on my thought, which compares... a badly constructed clock with the idea which I have of a ... well made clock, and is hence extrinsic to the things to which it is applied. (Descartes, Meditation VI, HR I 195)

As Descartes badly made clock exactly observes the laws of nature, although it does not show the time properly, so the patient's badly made or damaged pain protective mechanism strictly observes the basic neural and behavioral principles, although it does not show the threat properly and does not evoke the feeling of pain properly. From the internal point of view, the mechanism is put into action like any other normal mechanism. Namely, nociceptive multi-sensory neurons specialized for the detection of novel or potentially threatening visual objects start to fire when the instrument is seen as closely approaching the arm. As the visual presentation of the approaching instrument evokes the feeling of burning pain, the dangerous character of the visual stimulus is confirmed, and the specialized nociceptive multisensory neurons will fire more vigorously on the next presentation of the visual stimulus. There is nothing to decrease or inhibit their firing; but there would be nothing to decrease or inhibit the firing of these specialized neurons in the "normal" mechanism, if repetitive visual presentation of the needle closely approaching one's arm would invariably be followed by the feeling of pain inflicted by that needle. It is not improbable that, after such "visuo-doloric" training, the "normal" pain protective system would start to produce pain simply by the vision of the arm being approached by the needle. But the "normal" system could, luckily enough, be retrained, while the patients vicious system would resist any retraining. It is true that the patient has by himself found solution to one part of his problem: to the highly embarrassing involuntary withdrawal of his left arm whenever he would see the arm being approached by someone. He has solved this problem by simply tying his left arm to his belt. But the other part of the problem remained even after eighth months of rehabilitation: the burning pain and suffering that would appear as punishments when- 
ever he would watch his left arm being approached by someone. The fact that seeing can produce the feeling of pain need not anymore be completely mysterious to us. We have enough insight into the neural mechanism of pain that allows us to explain or come close to explaining how that extraordinary phenomenon is possible. But there is one problem related to that knowledge. Namely, knowledge is supposed to give us power or control over nature or natural phenomena; but, in the case of the vicious pain that we have been considering, it actually explains why we are powerless or helpless with regard to such pain; why the pain that the unfortunate patient is suffering cannot be put under control. We can solve and are close to solving the theoretical or intellectual puzzle of that vicious pain; however, the puzzle still remains whether and how it can be controlled. Till that puzzle is solved, our knowledge of pain mechanisms will seem modest compared to the amount of suffering that it still cannot put under control. 


\section{Dissociation Phenomena in Human Pain Experience}

As far as the possible biological and psychological dysfunctions or disruptions of the human pain protective system are concerned, we have till now considered only the most extreme or the most dramatic cases of such dysfunctions or disruptions: the total absence of pain and the complete maladaptive excessiveness and inadequacy of pain. But it often happens that people are only partially deprived of the gift of pain: that they can loose, due to lesions or surgical, pharmacological and cognitive manipulations, irreversibly or temporarily, just a portion of that gift or just one of the capacities that this gift consists of. And this observation will lead us to the consideration of the dissociation syndromes or phenomena in human pain experience. One example of such dissociations is the complete loss, fortunately reversible, of the capacity to display any behavioral reactions upon longlasting, intensive, painful stimulation, while the pain is felt in all its severity, is of excruciating character and totally unbearable. This radical dissociation of the behavioral component of pain from its affective and sensory components - the complete absence of former and the full presence of latter components - have experienced patients who were unfortunate enough to undergo major surgeries only upon the administration of curare which was, in the 1940's, considered by some physicians to be an efficient general anesthetic:

The patients were, of course, quiet under the knife, and made not the slightest frown, twitch or moan, but when the effects of the curare wore off, complained bitterly of having been completely conscious and in excruciating pain, feeling every scalpel stroke but simply paralyzed and unable to convey their distress. The doctors did not believe them. Eventually a doctor bravely submitted to the elaborate and ingenious test under curare, and his detailed confirmation of the subject's reports was believed by his colleagues: curare is definitely not any sort of anesthetic or analgesic. (Dennett, 1978, p. 433)

Namely, it has turned out that curare is a paralytic "that acts directly on all the neuromuscular junctions, the last rank effectors of the nerv- 
ous system, to produce total paralysis and limpness of all the voluntary muscles" (Dennett, 1978, p. 432). This is the reason why curarized patients could not in any way express their distress and agony, make any verbal complaints, display any avoidance or escape reactions, moan or make grimaces while experiencing excruciating pain. To the great relief of behaviorists, it should be remarked that the case of curarized patients cannot be taken as the conclusive evidence that one can be in pain without even having the slightest tendency, inclination or disposition toward any form of pain behavior. As we have seen, there is, indeed, in these patients a very prominent inclination, tendency or disposition to express their pain and to react to it; but, due to the muscular paralysis, they lack any means to realize that inclination, tendency or disposition; they are unable to translate them into verbal expression or into any bodily actions or reactions.

So, the question is, whether one can feel pain, in the strict sense of the term, and yet not have even the slightest tendency or disposition toward pain behavior? In other words, can one be completely indifferent to pain? But complete indifference to pain would also mean that one is not at all distressed by pain; that pain is not anymore the object of anxiety, fear or dread; that pain doesn't mean at all the threat or danger for the subject; that, finally, and what is hardest to understand, pain is not anymore disliked or experienced as inherently unpleasant. Such indifference to pain would actually mean that emotional-cognitive and behavioral components of pain experience would be dissociated from its sensory-discriminative components: that one would be able to feel pain, localize it, determine its intensity and qualitative character, and yet not react to it in any way. If that is possible, we would have the case of radical reactive dissociation syndrome in human pain experience. The case of the patients who have undergone the operation of pre-frontal lobotomy for the intractable, chronic pain, is usually taken as the paradigm case of this extreme reactive dissociation syndrome or of striking indifference to pain. Namely, it is characteristic for these patients that, after operation, they claim that their pain is still present, but that it doesn't bother them anymore or that they don't mind or care for it anymore. Here is the picture of the 
behavioral pattern and emotional-cognitive profile of one lobotomized patient depicted by Paul Brand:

I have had limited contact with lobotomized patients, but while in India I did see in one patient dramatic evidence of lobotomy's effect on pain. British woman from Bombay had for years sought relief from intractable vaginal pain. She tried every available pain-relieving pill, and even underwent surgery to sever nerves, but nothing helped.

A neurosurgeon on our staff had perfected a technique for lobotomy ... He would drill holes on both sides of the skull, run a wire through them, and then ... use the wire to slice through nerve pathways and separate the frontal lobes from the rest of the brain. He explained the risks to the woman, who immediately agreed to the surgery. She was ready to try anything.

By all measures, the lobotomy was a great success. The woman emerged from surgery completely free of the suffering that had shadowed her for a decade.

More than a year later I visited (her) in Bombay ... When I inquired about the pain, she said, "Oh, yes, it's still there. I just don't worry about it anymore." She smiled sweetly and chuckled to herself. "In fact, it's still agonizing. But I don't mind."

At the time it startled me to hear words about agony coming from a person with such a placid demeanor: no grimace, no groan, only a gentle smile. As I read about other lobotomies, however, I found she was displaying a very typical attitude. Patients report feeling "the little pain without the big pain". A lobotomized brain, no longer recognizing pain as a dominating priority in life, does not call for a strong aversive reaction. (Brand and Yancey, 1997, pp. 210-211)

Similar behavioral pattern and emotional-cognitive profile is to be found in the patients who underwent cingulotomy for chronic pain. Namely, it was assumed that cingulate cortex or cingulum, and particularly the anterior cingulate cortex, plays an important role in the affective-cognitive processing of pain: 
Therefore, it was attractive to postulate that transection of the cingulum might be of benefit in those clinical cases of intractable pain in which marked emotional factors appeared to contribute to the intolerable situation.

In this project (the) intent was to modify the patient's emotional response to the life-threatening situation which he faced so that his expression of fear and anxiety no longer augmented critically whatever pattern of organic pain was present to produce intolerable suffering. (Foltz and White, 1962, p. 89)

The immediate effects of cingulotomy on patient's affective and cognitive attitudes toward pain are described in the following way:

Immediate results from the lesions usually were apparent in the operating room. Continued verbal communication was maintained with each patient during the operation in order to evaluate to some degree his emotional state and degree of pain. A complaining, uncomfortable, apprehensive patient usually showed a dramatic change in demeanor at the time the lesions were made. The patient became tractable, agreeable and often showed a little vague disorientation. In 2 instances, this change occurred concomitant with simple insertion of the electrodes into the area of the cingulum prior to electrocoagulation. (Foltz and White, 1962, p. 92)

The long-lasting effects of this invasive psychosurgical method on the affective and cognitive attitudes toward ongoing, chronic pain are best to be seen in the description of one patient whose behavior and psychological profile are considered to be typical for cingulotimized patients:

Case I-3. M.W., 68-year-old white female, had been suffering from intense burning vaginal and perineal pain for over 2 years. ... The Department of Psychiatry felt she had "severe depression with maximum anxiety". The Department of Neurosurgery decided on cingulotomy because of the complaints of severe, incapacitating pain associated with complex emotional factors. 
After cingulotomy, the change was indeed striking. She ceased her continual whining complaints, began to take note on her external environment, and began to move about again. Within 2 weeks, she was up and walking for the first time in nine months. Shortly, she was able to leave the hospital, returned home, and resumed her housework activities. On questioning her, the pain was still present but did not concern her now. Over a $4 \frac{1}{2}$-year follow-up, her complaints of pain have not returned to clinical significance. (Foltz and White, 1962, pp. 9394)

The administration of morphine is said to have similar effects on pain perception as those that were observed in lobotomized and cingulotomized patients. Namely, under the impact of morphine the subjects also claim that the pain that they were suffering from is still there, that it continues to be pain, but that they no longer mind it. Because of this care-free attitude toward pain or care-free feeling of pain, some researchers were inclined to "describe the action of morphine (and some barbiturates) as reversible pharmacological leucotomy (lobotomy)" (Dennett, 1978, p. 430).

Now we have three cases that are usually mentioned in the relevant literature as typical cases of indifference to pain or as outstanding examples of radical reactive dissociation syndromes that are to be found in human pain experience. However, the indifference toward pain displayed in lobotomized and cingulotomized patients as well as in patients who are under the impact of morphine, is of limited scope; besides, it need not be directed at the very sensation of pain or the immediate threat that it imposes to the patient, but rather at the significance that subject is attributing to pain; finally, indifference to pain or care-free attitude toward it need not mean that the pain is not felt as unpleasant. Let me describe you one situation that strongly speaks in favor of the two last claims made with regard to the character of the indifference toward pain displayed by lobotomized, cingulotomized and morphinized patients. And this situation might well be familiar to you from your own experience. Namely, one can suffer from a long-lasting, dull and nagging pain that radiates towards the left arm and hand and is felt in the left upper region of the chest. This 
pain is not only unpleasant, annoying and distressing in the long run, but may evoke strong anxiety and fear because it can be taken as a sign of the impending heart infraction. Once the subject is reassured by his physician and told that the real cause of pain is the muscular inflammation, the anxiety and fear will be gone, and the care-free attitude toward the ongoing pain taken, although the pain will still be there and will still be felt as unpleasant. As far as the scope of indifference toward pain is concerned, it should be said that in lobotomized, cingulotomized and morphinized patients their carefree attitude is strictly limited to the ongoing pain, because they will vigorously react to any new pain inflicted by sudden intense noxious mechanical or thermal stimulation.

So, the question again is can one be completely indifferent toward pain in the sense of not minding at all for any pain inflicted by harmful stimulation and not having the slightest tendency or disposition toward any form of pain behavior? In other words, can one regularly feel pain upon harmful stimulation, localize it and assess its intensity and qualitative character, and yet on no such occasion dislike it, be distressed by it, have a fear or anxiety and try to avoid it? Is such pain without any painfulness possible? Can we at all think of such possibility? Are we able at least to conceive it or is it something that surpasses our imagination and is to be proclaimed as impossible on inconceivability grounds?

In order to give answer to these perplexing questions, I will invite the reader to try to imagine the following scenario. Namely, to try to imagine a person that, during thorough and daily repeated neurological sensory discrimination tests, feels pain and recognizes its quality, intensity and location whenever quite severe harmful stimuli are applied at any part of his body, but never finds it in the least unpleasant and never does anything about it: there are no withdrawal reactions whatsoever, no grimacing, no wincing, no complaints nor even the slightest tendency towards these common reactions to pain. Imagine further that this person, upon being challenged by the quite astonished neurologist to explicitly state whether he feels the corresponding harmful stimuli, says that he, indeed, feels them; that they hurt him a bit, but do not bother him and mean nothing to him. Moreover, and to 
even greater astonishment of the examiner, the person that you are supposed to imagine laughs or smiles while being exposed to obviously harmful or even torturing stimuli, and abruptly ceases to make this mockery of pain when the stimuli are discontinued. And to the utter surprise and relief of the neurologist involved in the examination, this person is to keep his friendly attitude toward him, never becoming angry at him, although the neurologist, and with good reasons, would normally be regarded as more of a torturer than an examiner. Add to this, that our imagined person is never to display any avoidance or protective reactions when approached by examiner's tools, like needles or hot probes, nor is he to show any signs of anticipatory anxiety, but is on the contrary sometimes quite ready to willingly offer his hands to painful testing. And the same absence of any motor and affective reactions is to be observed in him with regard to visual threats or verbal menaces: no flinch, no blink and no expression of fear. The imaginary person is to be conceived as being quite unaware of his plight, of his highly abnormal reactions to pain, and as being entirely unable to learn appropriate escape or avoidance reactions, despite the fact that he feels pain whenever an injurious or harmful stimulus is inflicted upon him. Finally, imagine what would happen to this person if he was ever to come into real life situations; for there is no doubt that the integrity of his body would be constantly threatened as he could, for instance, suffer serious hand burns without any escape or affective reactions.

One might claim that the imaginary case of pain without any painfulness, of pure pain reduced to totally indifferent sensory detection and discrimination of injurious stimuli, is not something that could ever really be found in human pain experience, but rather something that will at best remain, to use Eliot's wording, a perpetual possibility only in the world of speculation. Given our common experience of pain, this judgment may well seem to us to be the only proper one to make as far as the possibility of ever disclosing such pain in human kind is concerned. As Patrick Wall, one of the most distinguished figures in the field of pain research and theory, has put it: "I have never felt a pure pain. Pain for me arrives as a complete package. A particular pain is at the same time painful, miserable, disturbing, and so on. I 
have never heard a patient speak of pain isolated from its companion affect" (Wall, 1999, p. 149). Even more apodictic and exclusive statement along the same lines is to be found in Valerie Hardcastle's book The Myth of Pain: "Except under unusual circumstances, we cannot react to pain without also bringing forth a lot of additional baggage. There is no such thing as a simple pain state, nor a simple pain" (1999, p. 114).

But that there is such a thing as pure or simple pain, and that such pain is something that is really possible, stems from the fact that patients suffering from the so-called pain asymbolia experience exactly this kind of pain whenever they are harmfully stimulated at any part of their bodies. Namely, ever since the appearance of Schilder's and Stengel's seminal paper entitled "Schmerzasymbolie" (1928, pp. 143148), there are clinical reports on patients who feel pain that is literally deprived of any painfulness: who can "recognize pain but lack appropriate motor and emotional responses to painful stimuli applied anywhere on the body surface They may also appear insensitive to visual threats and to verbal menaces" (Berthier, Starkstein, Leiguarda, 1988 , p. 41). In other words, when I have initially invited the reader to imagine a person that, upon being harmfully stimulated, feels pain without, so-to-say, being in pain, I was not relying on fanciful thought-experiment devised to probe our deeply-entrenched intuitions about human pain experience, but rather on real life cases well-documented by the relevant clinical evidence. The fact that such pain may well appear to us to be inconceivable or to go beyond any comprehension is just to be taken as a proof that real life cases sometimes go over our imagination and that conceivability is not always a reliable guide to what is really possible. That is, the amazing and quite incomprehensible experiential report that our imaginary person was supposed to make when challenged by the astonished neurologist, as well as the smiling that he was meant to display on that occasion, has been actually made and displayed by one of the patients suffering from pain asymbolia: "To painful stimuli the patient responded with almost complete absence of protective and escape reactions. At the same time from her reports it could be gathered that she is sensitive to painful stimulus: 'I feel it indeed; it hurts a bit, but doesn't bother me; 
that is nothing', and so on. She smiles while saying this ..." (Pötzl and Stengel, 1937, p.180).

As far as the real import of this extraordinary statement and behavior of the asymbolic patient is at stake, one might say that it is just one more instance of that perplexing indifference to pain already found in lobotomized, morphinized and cingulotomized patients, and epitomized in their astonishingly carefree reports to the effect that something funny is happening to them: "The pain is still there, but it doesn't bother me". These reports are standardly taken as strong evidence that, due to reactive disassociation obtained by surgical or pharmacological manipulations, pain need not be experienced as unpleasant and that we should, accordingly, give up our deeply-entrenched intuition that it is something intrinsically or essentially disagreeable or disliked. So, within this conceptual and theoretical framework, the cases of pain asymbolia would mainly be treated as just more of the same: as an additional piece of evidence that the disassociation between sensory and affective aspects of pain is possible and that pain should thus be considered as only causally or contingently related to unpleasantness.

I will try to show that, on the contrary, pain asymbolia is to be regarded as the only clear-cut case in which quite severe pain is not at all experienced as unpleasant and in which there are no traces of any other aversive attitude towards it. Moreover, I will present conclusive evidence that, in contrast to the cases of lobotomized, cingulotomized and morphinized patients, the case of patients suffering from pain asymbolia should be considered as the only case of complete, thoroughgoing indifference to pain. In other words, I will claim that among the sensory-affective disassociation syndromes to be found in human pain experience, pain asymbolia is the sole one in which all affective reactions to pain are literally and for good lost, while the sensory aspect of pain is fully preserved. Indifference to pain, displayed in the statement "I feel pain, but it doesn't bother me at all", may well differ, from case to case, with regard to its scope and its proper object; as it will turn out, only in the case of pain asymbolia this attitude is consistently held towards any pain and is directed, not only to the possible significance or meaning of pain, but also to the 
very sensation of pain or the immediate threat that it poses to the subject. Pain asymbolia holds a unique place among reactive dissociation syndromes in yet another respect: namely, it is distinguished by total absence of any appropriate pain behavior and any tendency or disposition toward such behavior. As such, the case of pain asymbolia is to be taken as the most convincing evidence that pain without any appropriate pain behavior is possible. In other words, it can be taken as the strongest case against the behaviorist claim that at least the tendency toward pain behavior is necessary for pain. Namely, in asymbolia patients there is not the slightest tendency toward such behavior, although they feel pain when harmful stimuli are applied anywhere to their bodies. Moreover, these patients, when exposed to harmful stimuli and when feeling quite intense pain, often behave in ways that would by any standards be considered as just opposite to typical pain behavior: they smile to painful stimuli, welcome them and keep friendly attitude toward persons who are injuring them.

One might well appreciate the unique place that cases of pain asymbolia hold among the reactive disassociation syndromes to be found in human pain experience, but still claim that such freak possibilities, disclosed in clinical and brain studies, should be absolutely discounted. The reason for this harsh verdict being that these possibilities cannot in any way be relevant for our concept of pain or for our understanding of what it is to be in pain: that they should be treated just as extreme aberrations, residing far away from the core phenomenon, on its very edges or even going well beyond them. I will reject this verdict as unwarranted and claim that, on the contrary, thorough and careful consideration of the cases of pain asymbolia may well reveal to us the really complex nature of pain; its major constitutional elements; the proper role that they play in overall pain experience; the way that they work together, as well as the basic neural structures and mechanisms that subserve them. More specifically, I will argue that the consideration of these cases gives us the unique opportunity to clearly and fully see how much is missing and what is really missing in pain experience, once it is literary deprived of all its affective, cognitive and behavioral components, while its sensory aspect is kept intact. And this insight will, in its turn, make us realize 
that pain, when stripped of former components, loses all its representational and motivational force: that it comes to nothing in the sense that it is no longer a signal of threat or damage for the subject, and doesn't move (emotio) his mind and body in any way. If that is the case, it follows that the basic representational and motivational force of pain should not be sought in its sensory components, but rather in its affective, cognitive and behavioral dimensions. 


\section{Pain Asymbolia}

After these promissory notes related to the far-reaching conceptual and theoretical implications of pain asymbolia, I will in more detail present and assess the psychological profile, sensory capacities and behavioral pattern of patients suffering from this most radical reactive dissociation syndrome to be found in human pain experience. Although my exposition and elucidation of the cases of pain asymbolia will principally rely on the extensive and systematic study of this syndrome that was carried out by Berthier, Starkstein and Leiguarda (1988, pp. 41-49), I will also take into account other important reports and studies on asymbolic patients that basically support the major clinical findings of these authors. Now, in their own study, Berthier, Starkstein and Leiguarda have, for the purpose of precise and conclusive diagnosis, considered patients to have pain asymbolia "only if they were alert and cooperative; had no evidence of dementia, confusion or previous history of psychiatric disorder; had no deficit in pain perception; and had absent or inadequate motor and emotional responses to painful stimuli applied anywhere on the body surface" (1988, p. 42). After thorough neurological, psychophysical, neuropsychological and neuroradiological examinations, it has turned out that six patients with lesions in the specific areas of the brain complied to these quite stringent diagnostic criteria for pain asymbolia. The neurological examination of these patients consisted of the following tests:

Superficial pain was assessed through pinprick and thermal stimuli applied in the single, repetitive, or prolonged way over the face, neck, trunk, limbs, and perineal region. Deep pain was tested by heavy pressure to pretibial, sternal and supraorbital regions; passive hyperextension of fingers and toes; squeezing of calf muscles and Achilles tendons; and pinching of soft tissues on all four limbs. Motor responses (withdrawal and grimacing), emotional behavior, and autonomic reactions to noxious stimuli were examined. The responses to visual threats were also tested by presenting in either hemi-space aggressive gestures, such as pretending to slap the face, punch the nose, or 
prick the eyes with a needle. The degree of response - whether flinch, blink, or emotional reactions - to such menacing stimuli was recorded. In addition, the extent of reaction to verbal menaces was evaluated by announcing the application of a painful stimulus, with phrases such as "I am going to pinch you hard". Following this verbal threat, a noxious stimulus was given and the response assessed. (Berthier, Starkstein and Leiguarda, 1988, p. 42)

The relevant psychophysical examinations were carried out in order to measure the following variables:

(1) pain threshold, defined as the minimal stimulus intensity perceived as painful; (2) pain tolerance, defined as the point at which the stimulus was reported to become intolerable; and (3) pain endurance, defined as the arithmetical difference between pain tolerance and pain threshold. Left and right arms were independently stimulated. ... Bursts of 20 impulses/second lasting 2 seconds were delivered through two ring electrodes to the index finger. Stimuli were delivered every 3 seconds starting at $0-\mathrm{mA}$ and steadily increasing in intensity in $1-\mathrm{mA}$ increments. Patients were asked to tell when they began to perceive the stimulus as painful ... and when the stimulus became intolerable. After preliminary practice, five trials in each hand were performed and the average was calculated. (Berthier, Starkstein and Leiguarda, 1988, p. 42)

Patients have also gone through the battery of neuropsychological tests related to memory, intelligence, orientation capacities as well as tests for aphasia. Besides, clinical observations were made of other possible deficits, and all patients were exposed to computer tomography scanning for accurate estimation of lesion location and size. The pertinent neurological and psychophysical examinations have conclusively shown that in all six patients the ability to recognize pain upon noxious stimulation was fully preserved, but that they have consistently failed to display any affective and motor responses to painful stimuli. The conspicuous absence of such responses was also observed for visual threats and verbal menaces. Instead of displaying 
pain behavior, the patients have, in fact, behaved in ways that are just opposite to it. Here is the case report on one of these patients that I have actually used as a template for constructing the imaginary case of pain without painfulness:

In spite of apparently normal pain perception of superficial and deep pain, the patient showed a total lack of withdrawal responses. He tolerated prolonged pinprick or soft-tissue pinching in all four limbs, without adequate grimacing or defensive movements of his limbs. Neither did he show any response after sternal or supraorbital pressure, thus indicating a generalized defect. Such abnormal findings were constantly recorded throughout daily evaluations of pain. On occasion, the patient willingly offered his hands for pain testing and laughed during stimulation. He had no concern about the defect and appeared highly cooperative during pain evaluation. When the patient was asked specific questions concerning his sensory and affective feelings generated by a noxious stimulus, he tended to underrate the intensity of pain and made no adverse comments. In the course of pain testing session he seemed unable to learn appropriate avoidance responses. Verbal and visual threats also failed to produce protective or emotional reactions.

At times, his lack of withdrawal threatened his safety: his wife reported that he has accidentally suffered a serious left hand burn without escape or emotional reactions. (Berthier, Starkstein and Leiguarda, 1988, pp. 42-43)

The overall picture of pain asymbolia that emerges from this case report is, with some variations, characteristic of all other patients that Berthier, Starkstein and Leiguarda have studied, and this has led them to make the following collective portrayal of their six patients:

Although all 6 patients could adequately recognize painful stimuli and distinguish sharp from dull, all of them showed a lack of response to painful stimuli applied over the entire body. Neither superficial nor deep pain stimulation elicited a motor withdrawal, grimacing, or an appropriate emotional response ... One patient not only failed to show a withdrawal response but 
also exhibited a reaction of "approach" to the painful stimuli (i.e., he directed his limb toward the noxious stimuli). Inappropriate emotional reactions were common: 4 patients smiled or laughed during the pain testing procedure. This abnormal behavior ceased abruptly on discontinuing stimulation. All patients appeared quite unaware of their abnormal reactions and seemed unable to learn appropriate escape or avoidance responses. None of them became anxious or angry during the pain testing procedure; in fact, while all could recognize pain, none of them reported any unpleasant feeling. Patients showed normal autonomic reactions (tachycardia, hypertension, sweating, mydriasis) during the painful stimulation, but failed to react with flinch, blink, or adequate emotional responses to threatening gestures presented to both hemispaces. Five patients also failed to react to verbal menaces. (Berthier, Starkstein and Leiguarda, 1988, p. 43)

The psychophysical measurements of pain threshold, tolerance and endurance were carried out in three patients and the results of these measurements were compared to the results of the measurements of the same variables in five normal control subjects. "The preservation of pain perception, a critical requisite for diagnosis, was further confirmed in 3 patients through the measurement of pain threshold, which failed to reveal a significant difference between patients and a group of normal controls. As expected, all 3 patients showed significantly greater values for pain tolerance and pain endurance. The patients demonstrated a remarkably increased capacity to endure pain and to underrate the disagreeable stimulus characteristic" (Berthier, Starkstein and Leiguarda, 1988, p. 47). This tendency has been noticed, exactly sixty years earlier, by Schilder and Stengel in their patient Anna H.: actually, the first patient diagnosed in clinical records as suffering from pain asymbolia or the absence of appropriate affective and motor reactions to painful stimuli. Namely, when this patient was pricked in the left hand and asked by examiners whether it hurts her, they were faced with the following reply: "It hurts indeed, but I do not know what that really is. ... The patient again says: it hurts indeed a bit" (Schilder and Stengel, 1928, p. 151). The inclination to 
underestimate the intensity of the pain felt upon noxius stimulation and to make no adverse comments was even more prominent in the patient examined by Pötzl and Stengel: "II feel it indeed; it hurts a bit, but doesn't bother me; that is nothing', and so on" (1937, p. 180).

Schilder and Stengel have observed in their patient yet another kind of bizarre behavior that was, after six decades, also noticed by Berthier, Starkstein and Leiguarda in one of their asymbolia patients: namely, the reaction of "approach" to painful stimuli or of willingly offering limbs for further painful stimulation. According to Schilder and Stengel, there was absolutely no doubt that in their patient the pain sensation was present when she was strongly pricked. However, "the patient didn't evaluate the pain properly. This is revealed by the fact that she didn't at all or insufficiently withdrew from the painful stimulus, and that she even willingly offered herself to it" (Schilder and Stengel, 1928, p. 154). This same "approach", instead of "retreat", behavior was also notable in the patient with pain asymbolia studied by Rubins and Friedman: "He would not withdraw his hands or other parts of the body on painful stimulation (pinprick), although he could distinguish sharp from dull. He would even advance his arms for the painful pinprick" (1948, p. 559). So it seems that, at least in some patients suffering from pain asymbolia, it is not only the case that they fail to exhibit proper bodily reactions to painful stimuli, but that they exhibit those bodily movements or reactions that are exactly opposite to any normal reactions of people in pain or of people facing painful stimuli.

Another, even more inappropriate and more perplexing reaction of patients with pain asymbolia - that of smiling or laughing during painful testing - is exhibited on a much larger scale, and is to be considered as almost a kind regularity in the behavior of these patients. As reported by Berthier, Starkstein and Leiguarda, in their sample of six patients conclusively diagnosed for pain asymbolia, four were smiling or laughing during painful testing, and would abruptly bring it to an end when painful stimulation was halted. This completely weird and incomprehensible reaction to painful stimulation - something that is just opposite to normal distress, fear (fright) or anxiety accompanying these stimuli - was disclosed in the first clinical observation of 
pain asymbolia syndrome. Namely, Schilder's and Stengel's patient Anna $\mathrm{H}$. has reacted in the following way to the prick in the palm of her right hand: "The patient laughs contentedly, jerks the palm lightly, says 'oh hurts, that hurts', smiles on it, but stretches the hand further toward the examiner, and turns on all sides" (1928, p. 147) The same odd reaction was found out in Pötzl's and Stengel's patient during pain testing (1937, p. 180); and in a quite recently published article Ramachandran reports that the patient with the disorder called "pain asymbolia', which he has seen in India, "not only failed to experience the aversive quality of the pain but also started laughing in response to pinprick!" (1998, p. 1857) He admits that he has been completely amazed by such reaction to painful stimulus, and this has led him to put the question: "Is this not the ultimate irony: laughter in response to pain?" (Ramachandran, 1998, p. 1857) But this is not the only and far from final irony that is to be found in the responses of asymbolia patients to painful stimuli. As recorded by Berthier, Starkstein and Leiguarda, it was characteristic for all their patients that they were highly cooperative and that none of them became anxious or angry during the pain testing procedure, although they were daily exposed to repetitive, numerous and strong noxious stimuli. This amazing attitude was long ago observed by Schilder and Stengel in their patient Anna H.: "She never blamed the experimenter for inflicting pains to her and kept friendly, obliging attitude towards him even when the painful stimuli were numerous and very strong" (1928, p. 154). According to these authors, one testing session which consisted in pricking the needle in patient's left hand, and her declaring that it indeed hurts her a bit, has ended up in quite astonishing way: "The patient is cheerful, friendly and bids farewell to the examiner by curtsy (by performing knix)" (Schilder and Stengel, 1928, p. 151). Imagine paying in this way respects to somebody who would normally be considered as a person that one should immediately escape from and avoid forever. Isn't this the final irony: paying courtesy to your torturer or to somebody who is constantly inflicting pain to you?

But this astounding behavior may appear as less perplexing or more comprehensible if one takes into account the fact that patients with pain asymbolia are generally, as Berthier, Starkstein and Leiguarda 
have observed, unable to learn appropriate escape or avoidance responses to painful stimuli. However, the complete absence of such responses doesn't hold only for painful stimuli like stabbing of a sharp needle in the finger or applying the hot probe on the palm of the hand, for patients with pain asymbolia also fail to react and cannot learn to react to other threatening stimuli: aggressive gestures or verbal menaces. This was characteristic for almost all patients studied by Berthier, Starkstein and Leiguarda and is amply corroborated by other studies of pain asymbolia syndrome. Thus, Schilder and Stengel have explicitly pointed out that their patient "doesn't exhibit any appreciation of pain threat or of any threat whatsoever. Hitherto the inadequate reactions to very strong optical and acoustic stimuli are certainly to be taken into account" (1928, p. 154). Rubins and Friedman supply additional evidence for this general lack of threat appreciation or evaluation as it was also prominent in their patient: "In addition to the lack of reaction to painful body stimulation, when the examiner threatened her with an aggressive gesture, such as slapping her face, punching her nose, pinching her or even moving a pin toward her eyes, she did not flinch, blink or react emotionally" (1948, p. 557). In the same vein, what was abnormal in the case of Hemphill's and Stengel's patient suffering from word-deafness and pain asymbolia was not only the lack of normal reactions to painful stimuli, but also the lack of all reactions to any threatening stimuli in other modalities. When the patient was threatened with the prick to his hand or neck, "he made no effort to guard himself or to withdraw his head, nor did he show any instinctive combative reaction. Similarly, he appeared to be quite disinterested when a match was struck close to his face or eyes. He showed the same lack of reaction to unexpectedly loud noises or strong flashes of light" (Hemphill and Stengel, 1940, p. 256). What is characteristic of this behavior is that the stimulus is perceived and recognized, but that it is never appreciated for its meaning and affective valence, so that body and mind are not moved in any way but rest in the state of complete indifference or inaction. This is best illustrated by the following occurrence:

The patient was observed proceeding one morning along the main road of the hospital. He made no effort to get out of the 
way of a lorry behind him in spite of the loud warning of the horn. That he heard the horn and recognized its character is certain, for he admitted as much with considerable heat when he was forbidden, for his own safety, to walk alone on the main road. It was obvious from his action at the time that when he heard the motor horn he did not react as if it were a sound of warning. (Hemphill and Stengel, 1940, p. 256)

Like Hemphill's and Stengel's patient, one of the patients studied by Berthier, Starkstein and Leiguarda also suffered from pure word deafness, while the other one had a conduction aphasia similar to the patient recorded by Pötzl and Stengel. In all other patients from the sample of six patients neurobehavioral deficits, such as aphasia or Gerstmann's syndrome, were not prominent findings. Actually, common associated neurological and neuropsychological deficits were "unilateral or bilateral cortical sensory loss, rapidly resolving hemiparesis, unilateral neglect, and disorders of the body-schema" (Berthier, Starkstein and Leiguarda, 1988, p. 47). All patients, except one, have gone through monthly reevaluation for at least six months. The overall picture of pain asymbolia "remained unchanged in all 5 patients. ... In contrast, the 5 patients showed a substantial improvement in accompanying signs, particularly in hemiparesis, sensory loss, neglect, and body-schema disorders" (Berthier, Starkstein and Leiguarda, 1988, p. 46). Now, the interesting and indicative piece of evidence is the fact that, during the whole follow-up period of at least six months, only one out of five patients "was partially concerned about his condition and became fully aware and even astonished by his pathological laughter during painful stimulation" (Berthier, Starkstein and Leiguarda, 1988, p. 46). In the whole clinical literature on pain asymbolia that $I$ have perused, there is only one more instance of the patient who became aware of his ailment and his abnormal reactions to painful stimuli, and that is the one examined by Hemphill and Stengel. This patient was observed for a period that extended over half a year, and the symptoms of pain asymbolia did not change substantially. As has already been mentioned, there was no disturbance of sensibility in this patient, but "he did not show the normal reactions of withdrawal and defense to painful stimuli, nor to visual stimuli which 
usually give rise to such reactions, nor did he respond adequately to warnings of danger" (Hemphill and Stengel, 1940, p. 257). According to these authors, at one point the patient tried to rationalize this absence of reactions:

The patient soon discovered that the examiners were interested in the way he reacted to painful stimuli. He accordingly tried to explain his reactions by such expressions as: "I am not a man who cannot stand pain", or "I am used to that because I have worked on the road", or "Laborers are always hurting themselves; we don't take any notice of it." On the other hand, his wife assured us that he has always been susceptible to pain and had reacted violently whenever his children pricked or pinched him in play. (Hemphill and Stengel, 1940, p. 256) 


\section{How Is Pain Without Painfulness Possible?}

The cases of patients suffering from pain asymbolia allow us to say, without any contradiction, that one may feel pain without being in pain. Although pure pain or pain without any painfulness may seem to us to be almost inconceivable or incomprehensible, we must concede that it is possible because there is enough compelling clinical evidence that such pain really occurs or is really to be found in human experience. But, we may still wander how such outlandish pain - the pain that one finally smiles or laughs at - is possible or what makes it possible. According to Berthier, Starkstein, and Leiguarda earlier authors have considered neuropsychological deficits to be crucial factors in the pathogenesis of pain asymbolia. That is, this syndrome "has been variously ascribed to abnormalities of the body-schema, complex perceptual deficits, an altered relationship between body image and the awareness of pain, specific agnostic defects, and behavioral defects dependent on personality" (Berthier, Starkstein, and Leiguarda, 1988, p. 41). However, neuroradilogical examinations in which they have used computed tomographic images to accurately determine the location and extent of brain injury in their six patients, have led Berthier, Starkstein, and Leiguarda to come to the following conclusion:

... that lesion location was the most important factor associated with the development of the syndrome, as the insular cortex was invariably damaged in every patient. In fact, the development of the severe and persistent AP syndrome in 1 of our patients, following a discrete ischemic lesion in the posterior insula and parietal operculum, strongly suggests that the involvement of such structures may be sufficient for the production of the syndrome. (1988, p. 47)

Namely, the fact that exactly these structures were damaged may lend support to the claim that the characteristic behavioral pattern of patients with pain asymbolia could best be understood as a sensory-limbic disconnection syndrome. In other words, as somatosensory cortical areas responsible for the detection of sensory features of noxious 
stimulus are spared in asymbolia patients, they are quite able to recognize the modality, particular quality, intensity and location of the sensation evoked by such stimulus. However, the damage of the insula and parietal operculum may well disrupt the connections between sensory and limbic areas, so that the nociceptive stimulus would not be further processed for its emotional valence and the patient would have no emotional response to it. In this way, the lesion of particular anatomical sites could actually account for the characteristic dissociation between normal pain perception and adequate emotional responses in the patients with pain asymbolia. As Berthier, Starkstein, and Leiguarda have pointed out, this interpretation of pain asymbolia in terms of a sensory-limbic disconnection syndrome has been, more on speculative than evidential grounds, first put forward by Norman Geschwind in his famous article on disconnection syndromes in animals and man. And this is, in short, the path that Geschwind has taken in his hypothetical reasoning on the possible anatomical substrates of pain asymbolia:

Let as assume now that a patient develops a lesion not of the secondary sensory area but of the connection between it and the limbic system. It is conceivable that while the patient could still distinguish the qualities of the stimulus, he would have no emotional response to it.

My speculation would be that the connections from the secondary sensory area to the limbic system would go by way of insular cortex. The lesion causing pain asymboly would in fact spare the secondary sensory area but involve perhaps parietal operculum and insula, cutting of the connections to the limbic system. (Geschwind, 1965, p. 270)

As the results of the neuroradiological examinations of the six patients studied by Berthier, Starkstein, and Leiguarda uniformly point to the lesions of the parietal operculum and posterior insula, they have taken them as evidence that strongly supports Geschwind's hypothetical view about the possible anatomical causes of pain asymbolia and speaks in favor of his interpretation of this syndrome as the model of sensory-limbic disconnection syndrome. But, in order to make this 
interpretation of pain asymbolia empirically plausible and more precise, these authors had to rely on the new experimental evidence that was not at disposal to Geschwind when he was writing his article. And this new evidence was supposed to show that the relevant cortical structures really make the link between sensory and limbic areas, so that the damage to the posterior insula and parietal operculum could impair the processing of noxious stimuli by disrupting this connection. Such evidence Berthier, Starkstein, and Leiguarda have first found in Mesulam's and Mufson's (1985, pp. 179-226) demonstration that the posterior insula is reciprocally connected with the following sensory cortices:

(1) somatosensory - first and second somatosensory areas, areas 5 and $7 \mathrm{~b}$; (2) auditory - superior temporal cortex, granular, postauditory and parainsular cortices, first and second auditory areas; (3) visual - medial portion of inferior temporal gyrus, as well as paramotor cortex and high-order association areas on one hand and basomedial and lateral amygdaloid nuclei on the other. (Berthier, Starkstein, and Leiguarda, 1988, p. 48)

On the basis of these cortico-limbic interconnections and the role that posterior insula plays in them, Mesulam and Mufson have suggested that such sensory-limbic (posterior insula-amygdala) interaction is crucial for the assessment of the affective-motivational content of perceptual experience (1985, p. 216). Relying on this suggestion, Berthier, Starkstein, and Leiguarda have come to the following tentative conclusion:

Thus, if this sensory link is disrupted because of damage to the insula or closely connected structures such as area $7 b$, patients may still be able to identify modality-specific stimuli (somesthetic, visual or auditory) but may fail to react with appropriate motor responses or emotional tone, since they cannot attribute any significance to painful or menacing stimuli presented in these modalities. Following this interpretation, the behavior observed in most of our patients could be the result of a trimodal 
sensory-limbic disconnection. (Berthier, Starkstein, and Leiguarda, 1988, p. 48)

However, this interpretation leaves many questions that require much more precise and elaborated answers as far as the behavioral pattern typical for pain asymbolia and the possible anatomical substrates of this syndrome are concerned. The first question is related to the fact that patients with pain asymbolia fail to respond with appropriate motor and emotional reactions to painful stimuli applied anywhere on the body surface. That is, pain asymbolia is a bilateral deficit in the appreciation of painful stimuli. But neuroradiologocal examinations conducted by Berthier, Starkstein, and Leiguarda have disclosed that four of their patients had lesions of the insular cortex and parietal operculum on the left side, while two had them on the right side. So, why and how a unilateral insular or parietal opercular lesion could produce a bilateral deficit in the appreciation of painful or threatening stimuli? The second question that needs to be considered more thoroughly is related to the very nature of the behavioral deficit or syndrome that goes under the name of pain asymbolia. Is it a sensoryspecific behavioral syndrome as it is suggested by the above-mentioned interpretation? Or is it rather a more general multi-modal behavioral deficit due to the impairment and disruption of higher-level mechanisms of pain processing that integrate somatosensory nociceptive stimuli with other sensory modalities and with memory and learning so as "to provide an overall sense of intrusion and threat to the physical body and self" (Price, 2000, p. 1771). Closely related to this question are two more questions that certainly need to be answered if the behavioral pattern of patients with pain asymbolia is to be properly understood: Why they fail to respond not only to somatic noxious stimuli but also to visual threats? Why are they unable to learn appropriate escape or avoidance responses to threatening stimuli presented in any modality?

In order to give an answer to some of the questions raised above, Berthier, Starkstein, and Leiguarda had to take a closer look at the role that posterior insula and parietal operculum may play in the processing of noxious stimuli, for these were the brain structures that have been distinctively damaged in their sample of six pain asymbolia pa- 
tients. For these purposes, they relied on the available evidence coming from the electrophysiological studies of the relevant brain structures in monkeys that have been conducted by Robinson and Burton. I will pursue this line of evidence, but I will also take into account the much larger set of electrophysiological, neurobehavioral and lesion studies data that are nowadays available concerning the nociceptive responses of posterior insula and parietal operculum in monkeys. Besides, this set of data will be extended so as to cover the most recent data on the role that these brain structure may play in the processing of pain and, more generally, threatening stimuli in humans. But let us first consider in more detail the relevant electrophysiological data that Berthier, Starkstein, and Leiguarda have initially taken into account in order to explain certain puzzling anatomical and behavioral facts related to pain asymbolia.

What has principally attracted their attention in studies conducted by Robinson and Burton was the fact that these authors have examined in monkeys the responses of neurons belonging to the secondary somatosensory area and neighboring areas to noxious stimuli. Namely, it has turned out that few neurons responding to noxious stimuli were found in the secondary somatosensory area, but that both the granular insula and the area $7 \mathrm{~b}$ contained numerous neurons highly sensitive to such stimuli applied to either side of the body:

Area $7 \mathrm{~b}$ and granular insula were the major regions containing neurons that responded preferentially or exclusively to noxious or diffuse somatic stimuli. Brief noxious mechanical or noxious thermal stimulation affected $7.5 \%$ of the total sample from area $7 \mathrm{~b} . .$. Although the neurons that responded to noxious, thermal, or diffuse stimulation were found throughout area $7 \mathrm{~b}$, there were some indications that these neurons were clustered in certain regions, and they were found within the "whole body" representation within area $7 \mathrm{~b}$. (Robinson and Burton, 1980, pp. 99-100)

The other interesting and important result of Robinson's and Burton's electrophysiological study was their discovery that within area $7 \mathrm{~b}$ there also exist neurons that respond to visual stimuli. But what was 
even more important was their finding that among these neurons there were some which responded specifically to threatening visual stimuli:

Some neurons responded when the animal was shown an undesired, noxious object, such as a pin that has just been used to deliver a slightly painful prick. These responses stopped, following repeated visual presentation of the pin, or after a lapse of 10-15 minutes. However, a response could be elicited by showing the pin if this object was again used to apply a painful stimulus. Visual presentation of neutral objects, such as a tuning fork, or paint brush, did not elicit responses from these neurons. (Robinson and Burton, 1980, p. 101)

Robinson's and Burton's findings were in three respects significant and helpful to Berthier, Starkstein, and Leiguarda. First, their results have shown that radical impairment of pain processing may, indeed, stem from the damages of granular insula and parietal operculum, for numerous neurons highly sensitive to noxious stimuli were exactly found in these regions, and not in the secondary somatosensory area. And as damages in pain asymbolia patients were predominantly insular and parietal opercular damages, they would be the mayor cause of the appearance of this syndrome, and not damages to the secondary somatosensory area as was earlier claimed by Biemond (1956, pp. 221-231). Besides, the fact that neurons specifically responsive to visual threatening stimuli were found in granular insula and parietal operculum would give a clue why asymbolia patients with damages to these areas failed to react to such stimuli. As similar neurons were not disclosed in secondary somatosensory area, the unresponsiveness of pain asymbolia patients to visual threatening stimuli would remain completely mysterious if that syndrome would have to be accounted by the damage of that area. Finally, the evidence that there are in granular insula and parietal operculum neurons highly sensitive to noxious stimuli applied to either side of the body - that they are bilaterally activated - would cast light on how a unilateral insular or parietal opercular lesion could give rise to bilateral deficit in the appreciation of painful or threatening stimuli. 
The electrophysiological evidence for the existence of neurons in the cortical area $7 \mathrm{~b}$ in monkeys that are preferentially responsive to noxious stimuli as well as for neurons that are highly sensitive to visual threatening stimuli, supplied by Robinson and Burton, was confirmed and in several important respects, pertinent for the understanding of pain asymbolia, further developed by Dong et al. (1994, pp. 542-564). Namely, in their sample of 244 neurons that were isolated in the trigeminal region of cortical area $7 \mathrm{~b}$ and studied, through microelectrode recording, for their responsiveness to somatosensory and visual stimulation, Dong et al. have found out that thermal nociceptive neurons "made up 9\% (21 of 244) of the neurons that had somatosensory response properties ... Thermal nociceptive neurons responded either exclusively to noxious thermal stimuli (highthreshold thermoreceptive, HTT) or differentially to non-noxious and noxious thermal stimuli (wide-range thermoreceptive, WRT)" (1994, p. 542). This group of neurons was, in its turn, particularly examined so that their stimulus intensity-response functions could be precisely determined and correlated to the stimulus intensity-escape frequency functions obtained while monkeys were performing the pain tolerance task. As far as the sensitivity of thermal nociceptive neurons, both of HTT and WRT kind, to the increases of noxious thermal stimulus intensities is concerned, it has turned out that these neurons can be functionally differentiated "into subpopulations that did encode (EN) and did not encode (NE) the magnitude of noxious thermal stimuli intensities" (Dong et al., 1994, p. 542). This was measured by applying calibrated thermal stimulation to the faces of monkeys and recording the stimulus intensity-responses of the relevant neurons so as to assess whether they increased or decreased their mean discharge frequency to increased noxious temperatures. Shifts in thermal stimuli intensities were made from an adapting temperature of $38^{\circ} \mathrm{C}$ to temperatures ranging from 40 to $51^{\circ} \mathrm{C}$. All thermal nociceptive neurons that were examined have responded reliably and effectively to temperature of $44^{\circ} \mathrm{C}$ : some of them (HTT) just at that stimulus intensity level and some (WRT) at that level and the levels below it. The intriguing and important question was how this group of neurons would respond to temperatures above $44^{\circ} \mathrm{C}$, and particularly to temperature 
shifts from 47 to $51^{\circ} \mathrm{C}$; for it is exactly at the temperatures around $48^{\circ} \mathrm{C}$ that nerve substance begins to suffer injury or would be frankly damaged if the stimulus was prolonged or continued. The relevant measurements have given the following results:

... that the two subpopulations of thermal nociceptive neurons, WRT-EN and HTT-EN, graded noxious thermal stimulus intensity by increasing their mean discharge frequency in a monotonic manner to increased noxious temperatures. In contrast the mean discharge frequencies of WRT-NE and HTT-NE neurons decreased precipitously at higher noxious plateau temperatures ... Therefore these two other subpopulations of thermal nociceptive neurons ... did not reliably encode the magnitude of noxious thermal intensity by graded discharge rates. (Dong et al., 1994, p. 548)

Now, the shifts in temperature intensities of the stimulus that WRTEN and HTT-EN thermal nociceptive neurons reliably graded by increasing their mean discharge monotonically, were exactly the shifts from 47 to $51{ }^{\circ} \mathrm{C}$ : that is, the temperature intensities at which nerve substance starts to be damaged or would be damaged if the stimulus was not discontinued. The fact that these neurons are physiologically specialized to effectively encode harmful thermal stimuli as well as the degree of their harmfulness, may well lead us to surmise that, from the functional point of view, they are perfectly and distinctively suitable to play the role of neurons that alarm an organism to the presence of threatening or potentially threatening thermal stimuli so as to make it to avoid them or escape from them. This was perhaps the reason why Dong et al. have carried out behavioral studies on their sample of three monkeys, and tried to see whether there is a significant correlation between the stimulus intensity-escape functions obtained during the performance of an appetitive tolerance-escape task and the stimulus intensity-response functions of the relevant physiologically specialized subgroup of thermal nociceptive neurons of area $7 \mathrm{~b}$. The appetitive tolerance-escape paradigm was used for the measurement of thermal pain tolerance because it presented a conflict between two reinforcers to the monkey: 
... that is, a choice between a positive reward (acquiring protein-fortified fruit sauce or fruit-flavored water) and negative reward (terminating noxious thermal stimulus). Such a model contains a number of desirable features for assessing behaviors evoked by stimulus intensities above pain threshold. ...: (1) it ensures that subjects adopt conservative biases for aversive responding during the stimulus period and it operates within a stimulus intensity range that activates nociceptors when the pain tolerance threshold (50\% escape responding) is met or exceeded; (2) it eliminates any avoidance component by allowing the experimenter to determine the sequence of stimulus intensities; and (3) it allows comparisons of escape frequency (percent) and latency to stimulus intensities and estimates the growth rate ... of pain magnitude from tolerance threshold to nearly $100 \%$ escape responding. (Dong et al., 1994, p. 543)

The measurements of thermal pain tolerance in three monkeys who were performing appetitive tolerance-escape tasks have conclusively shown that there is, indeed, a significant correlation between "noxious thermal stimulus intensity and the mean discharge frequency of individual WRT and HTT neurons and between the same intensity and mean escape frequency (thermal pain tolerance) ..." (Dong et al., 1994, p. 549). Namely, for three monkeys "the pain tolerance thresholds $(50 \%$ escape responding) and corresponding $95 \%$ confidence intervals were $48.3 \pm 3.8^{\circ} \mathrm{C}, 47.2 \pm 1.6^{\circ} \mathrm{C}$, and $45.1 \pm 3.2^{\circ} \mathrm{C}$, respectively" (Dong et al., 1994, p. 547). And it is obvious that the thermal stimuli intensities which evoked 50 percent escape responses in monkeys closely approximated the stimulus intensity response functions of the HTT-EN and WRT-EN neurons in area 7b: for the thermal intensity shifts which these neurons graded by increasing their mean discharge frequency in monotonic manner were precisely the temperature intensity shifts from 47 to $51^{\circ} \mathrm{C}$. For instance, in the case of one monkey, HTT-EN neurons encoded the temperature intensity increase to $48^{\circ} \mathrm{C}$ that evoked over 50 percent escape responses and their peak mean discharge frequency was at the thermal stimulus intensity of $49^{\circ} \mathrm{C}$ that evoked almost 90 percent of escape responding. It should also be said that the stimulus intensity-response functions of 
the HTT-NE and WRT-NE neurons - that is, of the neurons that did not reliably encode the magnitude of noxious thermal intensity by graded discharge rates did not approximated significantly stimulus intensity-escape frequency functions.

It is generally acknowledged that, in contrast to pain perception threshold, the pain tolerance threshold is not closely related to the sensory-discriminative component of pain, but much more to its evaluative and affective-motivational components. If that is the case, the fact that the subpopulation of $7 \mathrm{~b}$ area nociceptive neurons (HTTEN and WRT-EN) has stimulus intensity-response functions that are closely and significantly correlated to stimulus intensity-escape functions, would strongly speak in favor of the assumption that the activity and distinctive stimulus-response properties of this physiologically specialized group of neurons are much more related to the evaluation or appreciation of the threatening or potentially threatening nature of the stimulus, of its aversive character or its affective-motivational valence, than to the very discrimination of the stimulus. To put this assumption into more psychological terms, the firing and distinctive response properties of the relevant group of neurons would correspond more to the conscious appreciation of pain than to the perception of pain. That this might well be the case, was further corroborated by Dong et al., in a complementary lesion study of one monkey that had trauma to the posterior parietal cortex. Namely, that brain area was damaged during the period when the monkey has taken part in the electrophysiological and behavioral studies described above, and that has given the unique opportunity to examiners to investigate lesion consequences with respect to electrophysiological and behavioral responses and to compare these responses to the responses of the same kind that the animal had before lesion. Actually, the brain trauma in the relevant area was discovered after Dong et al. had noted, over several daily electrophysiological and behavioral recording sessions, "a sharp reduction in the number of cells ... with resting discharges or with cutaneous receptive fields and ... a precipitous decrease in escape frequency to noxious thermal shifts" (1996, p. 580). The trauma itself was due to the unilateral focal cerebral compression that was centered over the inferior lobule of the left posterior 
parietal cortex and parietal operculum and almost eliminated escape behavior to noxious temperatures applied to the skin on the contralateral maxillary face region:

Multiple comparisons of the mean values showed that before brain trauma, escape frequencies associated with noxious temperatures of $47^{\circ}$ to $51^{\circ} \mathrm{C}$ were significantly greater... than the escape frequencies associated with temperatures of $43^{\circ}$ to $46^{\circ}$ C. After brain trauma, escape frequencies for temperatures of $47^{\circ}$ to $51^{\circ} \mathrm{C}$ were significantly reduced ... from pre-trauma escape frequencies for the same temperatures and were not significantly different then escape frequencies for temperatures of $43^{\circ}$ to $46^{\circ} \mathrm{C}$ ". (Dong et al., 1996, p. 583)

For instance, at critical noxious temperature intensity of $48^{\circ} \mathrm{C}$ monkey's escape responses were below 10 percent and went even further down to almost complete disappearance when shifts in thermal stimulus intensity were made from $48^{\circ}$ to $51^{\circ} \mathrm{C}$. However, additional tests were designed to examine whether the damage to the relevant brain area would also lead to the impairment of monkey's sensorydiscriminative capacities or its ability to detect and discriminate noxious thermal stimuli. For these purposes, the monkey was trained to detect the offset of noxious thermal shifts, applied to the contralateral or ipsilateral maxillary region of the face, from the $48^{\circ} \mathrm{C}$ plateau temperature to $38^{\circ} \mathrm{C}$ temperature. And it has turned out that nearly all downshifts "from $48^{\circ}$ to $38^{\circ} \mathrm{C}$ were detected (button release) within $8 \mathrm{~s}$ on the contralateral or ipsilateral face. ... The same result outcome was obtained when successful trial completion required offset detection of non-noxious thermal shifts $\left(42^{\circ}\right.$ to $\left.38^{\circ} \mathrm{C}\right)$ applied to the contralateral face" (Dong et al., 1996, pp. 583-584).

What these further results have shown is that trauma to the posterior parietal cortex and parietal operculum may well leave the sensorydiscriminative capacities of the animal completely intact, although it has led to the thorough impairment of motivational and affective behaviors. In other words, they have demonstrated that the capacity of the animal to detect and discriminate noxious thermal stimuli with regard to their very appearance and quality may be fully preserved, 
while the ability to appreciate their threatening or potentially threatening nature, their aversive or affective-motivational valence may be almost entirely eliminated. And one should remember that such selective deficit in pain experience and pain behavior was exactly the distinctive mark of pain asymbolia syndrome. Namely, patients suffering from pain asymbolia were quite capable to detect noxious stimuli, to discriminate their quality, and to feel pain on that account, but were, like the monkey studied by Dong et al., incapable to appreciate the threatening nature of such stimuli and display any avoidance or escape behavior. Compared to control subjects, their pain tolerance threshold was highly increased and they systematically tended to underestimate the intensity of the injurious stimuli inflicted upon them. And the point is that pain asymbolia patients had lesions or damages in approximately the same areas of the brain as those that were found out in the brain areas of the monkey studied by Dong et al. Actually, this important lesion and behavioral animal study provides us with additional and independent evidence that damages to the posterior parietal cortex and parietal operculum may, indeed, lead to selective impairments in pain experience and pain behavior. Thus, they strongly speak in favor of the claim made by Berthier, Starkstein, and Leiguarda that lesion location was the most important factor associated with the development of pain asymbolia syndrome: that is, of their claim that the distinctive disassociation between sensory-discriminative and affective-motivational components of pain to be found in pain asymbolia patients may best be explained on neural grounds.

This conclusion is also supported by most recent lesion studies in humans whose primary aim was to determine the impact of damages involving parasylvian cerebral cortex on pain perception thresholds and pain tolerance thresholds of patients who had trauma to this region of the brain. The MRIs of six patients were evaluated so that it could be determined "to what extent the following cerebral regions were involved in the lesion: anterior insula, posterior insula, retroinsula and parietal operculum" (Greenspan et al., 1999, p. 273). This evaluation has shown that each patient's lesion involved at least two of these regions, and that has enabled the examiners to compare more 
precise lesion locations with the results of the measurements of pain perception thresholds and pain tolerance thresholds in patients. It has turned out that patients with elevated pain perception thresholds had lesions restricted to parietal operculum and posterior insula while the parietal operculum was spared in patients that showed no evidence of abnormal pain thresholds. Of the four patients that have gone through the pain tolerance tests, only two have displayed greater or increased tolerance, and theirs were the two lesions that involved a large part of the insula. As the lesion picture of six pain asymbolia patients studied by Berthier, Starkstein, and Leiguarda involved, as a rule, that region of the brain, the authors of the new lesion study presented here have come, under the premise that pain tolerance test is related more to the affective/motivational aspects of pain, to the following conclusion:

Our results and Berthier's results support the idea that the insula's role in nociceptive information processing is not related to pain threshold. Rather, the insula is more likely to have a role in the more affective and motivational aspects of pain. (Greenspan et al., 1999, p. 281)

The electrophysiological single-cell recording study of the response properties of nociceptive neurons in area $7 \mathrm{~b}$ of monkeys, carried out by Dong et al., may in yet another respect be important for the understanding of the neural grounds of pain asymbolia syndrome, and particularly for the explanation of the fact that patients suffering from this syndrome fail to respond not only to somatosensory, but also to visual threatening stimuli, and are, moreover, incapable to learn avoidance behavior to such stimuli. Namely, as the authors of this study have themselves remarked, the thermal nociceptive neurons in area $7 \mathrm{~b}$ had one outstanding and uncommon feature:

A prominent and unusual feature of WRT and HTT neurons in area $7 \mathrm{~b}$ was their multi-modal properties (responsiveness to both thermal and mechanical stimulation) (12 of 13 neurons tested) and their multi-sensory properties (responsiveness to both somatosensory and visuosensory stimulation) (7 of 21 total neurons). Visuosensory stimuli that evoked the highest mean discharge rate from multi-sensory neurons were the ap- 
proach and less often the withdrawal of novel or threatening objects (i.e. syringe and needle) along trajectories to and from the face. The most effective trajectories of these visual targets were aligned with the most sensitive portion of the thermal and/or mechanical cutaneous receptive field. The mean discharge rate was decreased by iterative presentation of the same novel object. ... Familiar objects such as the experimenter's finger ... and "moving lights" toward and away from the face (motion in depth) were ineffective visuosensory stimuli. (Dong et al., 1994, p. 550)

Particularly interesting for our purposes is the case of an HTT-EN neuron with multi-modal and multi-sensory response properties, for it has already been found out that stimulus intensity-response frequencies of thermal nociceptive neurons that encode the magnitude of noxious thermal stimulus intensities are closely correlated to stimulus intensity escape frequency functions. And this close correlation has led us to attribute the behaviorally significant function to these neurons related to the detection of the threatening or aversive character of the thermal noxious stimulus and the inducement of escape responses. So let us present in more detail the distinctive multi-modal and multisensory response properties of an HTT-EN neuron and see whether they might also play the biologically and behaviorally important role for the organism:

The maximum visuosensory response was evoked by a threatening visual target (i.e., syringe and needle) that approached the face along a trajectory that was aligned with the contralateral maxillary region and was held close to the same region. ... Because visuosensory responses may contaminate responses evoked by mechanical or thermal stimulation of the contralateral maxillary region, the monkey was temporarily blinded to the approach of the experimenter's hand and to the thermal probe resting on the face. ... As shown ... moving the thermal probe across the skin, brushing the hairs, and application of pressure and pinch to the skin reduced the background level of discharges in this neuron. Thermoreceptive responses ... were evoked by applying graded thermal shifts ... to the contralateral 
maxillary region from an adapting temperature of $38^{\circ} \mathrm{C}$ to temperatures ranging from 45 to $51^{\circ} \mathrm{C}$. The thermal S-R function constructed for this neuron in area $7 \mathrm{~b}$ showed the grading of noxious thermal intensities. ... The maximum peak discharge frequencies in response to noxious thermal and visual stimulation were approximately equal ... (Dong et al., 1994, pp. 550551)

As far as the significance of the results presented above is concerned, the first thing that one should notice is that they strongly confirm Robinson's and Burton's earlier finding that in the area of granular insula and parietal operculum of monkey's brain there are some neurons which respond specifically to threatening visual stimuli. As we have said, this finding gave us a first clue to explain how pain asymbolia patients, who had lesions in exactly these areas of the brain, could systematically fail to respond to such stimuli by protective or avoidance behavior. However, it has not given us enough clues to understand in more precise and detailed terms why that should happen; why these patients were, although repeatedly exposed to visual threatening stimuli, unable to learn to avoid such stimuli or protect themselves from them; and, finally, why they have in some cases shown the tendency to expose themselves willingly to injurious stimuli or to approach them without fear.

The more elaborated and expanded electrophysiological and behavioral study, conducted by Dong et al., may well provide us with plausible answers to these questions, because these authors have disclosed that neurons that best responded to threatening visual stimuli were also distinctively responsive to somatosensory niciceptive stimuli: that, for instance, the same neuron that responded to the approach of the syringe or needle to the face or when they were held in close proximity to the face would, upon the application of the hot probe at the face, best respond to noxious or damaging thermal stimuli, encoding reliably thermal stimuli intensity shifts from $47^{\circ}$ to $51^{\circ} \mathrm{C}$, and reaching its highest mean discharge frequencies (rates) exactly at thermal stimulus intensities that have evoked the most frequent and consistent escape responses in monkeys. Besides, Dong et al. have found out that the nociceptive neuron with these multi-sensory prop- 
erties would respond to the threatening visual stimuli only if the syringe or needle were directed at or positioned close to its cutaneous (skin) receptive field: that is, directed at or positioned closely to the field on the skin of the face from which the nociceptive neuron would be activated if pinpricking or heat contact were applied at the skin of the face. Now, the fact that nociceptive neurons in area $7 \mathrm{~b}$ are multisensory - that they respond both to somatosensory and visuosensory stimulation - points to the conclusion that the neural organization of this region of the posterior parietal cortex "appears to be that of integrating nociceptive inputs with other sensory inputs in a manner that conveys information about the overall degree of threat presented to an organism" (Price, 2000, p. 1771). Of course, this cross-sensory integration purported to provide, "an overall sense of intrusion and threat to the physical body and self" (Price, 2000, p. 1771), would have to play a role in or to be closely related to memory and learning. That this may well be the case is supported by the observation, to be found in the study of Dong et al., that the mean discharge rate of nociceptive multi-sensory neurons was decreased by iterative presentation of the same novel object, as well as by the following remark made by Robinson and Burton: that responses to threatening visual stimuli, such as a pin, "stopped, following repeated visual presentation of the pin, or after a lapse of 10-15 minutes. However, a response could be elicited by showing the pin if this object was again used to apply a painful stimulus" (Robinson and Burton, 1980, p. 101). What this shows is that the neural mechanism which consists of nociceptive multi-sensory neurons is perfectly apt to leave traces in the memory about the threatening or damaging nature of the stimuli and to ground associative learning with regard to such stimuli. As we have already seen, the mechanism actually has two basic capacities that make it suitable to play this role. First, multi-sensory nociceptive neurons have the capacity to encode reliably and effectively the threatening or damaging nature of the noxious somatosensory stimuli, such as pinprick or hot probe contact, when they are applied at the face and to induce avoidance behavior. Second, they are tuned to respond to novel objects whenever they are directed at or positioned close to the face of an organism: that is, when the target location or direction of 
motion within the visual receptive field is spatially aligned with the cutaneous receptive field (Price, 2000, p. 1771). As Dong et al. have noted:

Such a neural ensemble in area $7 \mathrm{~b}$ could conceivably work as a spatially and temporally coordinated unit to provide continuous sensory information about (1) the general location of the novel and potentially noxious stimulus in extra-personal space and its rate of motion in depth ... and (2) the general location of a noxious stimulus in personal space and its change in magnitude. Such dynamic visual-somatic information about an approaching noxious stimulus and impending tissue damage, respectively, may be necessary for directing motor adjustments ... to minimize body exposure and contact with the offending stimulus. (1994, p. 561)

So, when the object such as a needle or a glowing match is for the first time visually presented in the vicinity of monkey's face, it will evoke the response of nociceptive neurons in area $7 \mathrm{~b}$ and make them, so to say, ready for the assessment of the potentially noxious or threatening significance of the incoming stimuli. For that to happen, the needle or glowing match would have to approach the face surface along a precise trajectory that would ideally bring the stimuli in contact with the cutaneous receptive field of the nociceptive neuron. Once the face surface is, on that precise point, encroached by the needle or the glowing match, the nociceptive neuron will encode the harmful or damaging nature of the stimuli and induce escape behavior. This will lead to the integration of the somatosensory noxious stimulus with the visuosensory stimulus and the latter stimulus will be associated with potentially noxious or threatening significance and become apt itself to evoke escape behavior. Repeated approach and encroach of the needle or glowing match would strengthen this associative tie and, thus, foster the threatening significance of the visual stimuli as well as the frequency of escape from it. That this may well be so is supported by the fact that, in the case of nociceptive multisensory neurons "the maximum peak discharge frequencies in response to noxious thermal and visual stimulation were approximately equal ..." (Dong et al., 1994, pp. 550-551). However, the mean dis- 
charge rate of nociceptive multi-sensory neurons would dramatically decrease or their responses would be stopped by the repeated approach of the needle of glowing match to the face surface, if this iterative presentation of corresponding visual stimuli has not been followed by the pinprick or heat contact. In other words, the potentially noxious or threatening significance would be disassociated from the corresponding visual stimuli and they would not anymore evoke escape responses. As Robinson and Burton have observed, the association could be reinstated if the needle or glowing match pin were again used to apply a noxious mechanical or thermal stimulus such as pinprick or hot contact.

Now that we have an insight into how the neural mechanism of nociceptive multi-sensory neurons in area $7 b$ works, we are in the better position to understand why the trauma to the parietal operculum and posterior insula may well deprive an organism of the capacity to respond to threatening visual stimuli and learn avoidance behavior to such stimuli. Namely, due to the trauma in the relevant brain area an organism would not be anymore able to integrate visuosensory stimuli with noxious somatosensory stimuli. In other words, the pinprick or heat contact that followed the visual presentation of needle or glowing match directed at or held close to the face surface, would simply not evoke the response of nociceptive neurons that encode the damaging or destructive nature of noxious mechanical and thermal stimuli and induce escape behavior. Thus, the visual presentation of needle or glowing match would not be associated with damaging or destructive stimuli and would neither acquire the significance of potentially noxious or threatening objects nor the strength to elicit escape behavior by itself. An organism would simply miss the basic neural mechanism by which it could learn the potentially noxious or threatening significance of visual stimuli and avoidance behavior to such stimuli. As pain asymbolia patients have suffered trauma in the region of parietal operculum and posterior insula which are exactly the seat of this mechanism, one could explain along the same lines their inability to attach potentially noxious or threatening significance to visual stimuli and learn to avoid such stimuli. The complete absence of this capacity would also make it more understandable why they sometimes even 
show the tendency to approach harmful objects or willingly expose themselves to them. 


\section{Conceptual and Theoretical Implications of Pain Asymbolia}

Now that we have, on neural grounds, explained how pain without painfulness is possible, let us try to see whether and in what respect this extraordinary phenomenon can be relevant for our understanding of the very concept or the very nature and structure of pain. As far as these conceptual and theoretical implications are concerned, it seems that there are at least two important lessons to be learned from the consideration of pain asymbolia syndrome. The first lesson being that pain, although appearing to us as simple, homogenous experience, is actually a complex experience comprising sensory-discriminative, emotional-cognitive, and behavioral components which commonly go together, but may well be disconnected and thus exist, to our great astonishment, separately. The second lesson being that pain, once deprived of all its affective, cognitive, and behavioral components, loses all its representational and motivational force: that it is no longer a signal of threat or damage and doesn't move ones mind and body in any way. It becomes a blunt, inert sensory appearance with no power to galvanize the mind and body for flight or fight, and doesn't serve anymore its primary biological function. As Denny-Brown has remarked: "for such patients (i.e. those with pain asymbolia) that we have seen can feel pain and can discuss it, though it is not of any biological importance to them" (1962, p. 244). In other words, for these patients pain does not play anymore the role of the biological system that protects an organism by, as the meaning of the original Latin word poena says, punishing it for doing something that may injure it or for doing something that may worsen the already inflicted injury; and, as every punishment, reminding it or making it learn that it should avoid doing something or avoid certain objects or situations. On the contrary, the pain that they feel upon being harmfully stimulated comes, in their own wording, to nothing or, at best, to something that they consistently smile or laugh at during repeated pain testing procedures. 
But how can one react to pain by smiling or laughing? What would one actually smile or laugh at? The principal reason or cause why pain asymbolia patients can and do smile or laugh at pain that they feel upon being harmfully stimulated is to be found in the fact that they are not experiencing or perceiving it as a threat, danger, or damage to the integrity of their bodies; actually, by smiling or laughing at it they are dismissing it as sign of physical damage or at least threat to the physical well-being. For them, pain is a mock threat or mock danger, because in their case nothing horrible, frightening or awful is experienced in the pain that they feel upon being, for instance, severely pinpricked. But pinpricks, particularly severe ones, are expected to evoke strongly aversive or frightening sensations; as the pain that asymbolia patients feel on such occasions does not correspond to these expectations, the incongruity between what is expected and what is experienced or perceived makes them smile or laugh at the pain that they feel when severely pinpricked. That the smiling and laughter displayed by asymbolia patients is specifically directed at the peculiar character of the pain that they feel during noxious stimulation, and not elicited by some other factors, is strongly supported by the fact that this strange behavior ceased abruptly on discontinuing stimulation. However, asymbolia patients may smile or laugh during pain testing not only because the pain that they feel upon harmful stimulation is perceived by them as mock threat or mock danger. In their case, smile and laughter may also be understood as communicative acts directed towards neurologists who are daily and repeatedly exposing these patients to injurious stimuli and would normally, as we have already said, be considered more as torturers than examiners. In other words, smile and laughter may well be the expression of reassurance in social transactions: telling to neurologists that their needle pricks or punches are experienced as mock aggression, as no real danger, so that they have actually not done anything wrong. This interpretation of the bizarre smiling or laughter displayed by asymbolia patients during pain testing is supported by the fact that these patients never get angry at examiners; keep a cooperative and friendly attitude towards them during the whole testing period and sometimes may even pay their respects by curtsey. 
As we have seen, the fact that asymbolia patients consistently smile during the whole pain testing procedure is basically the outcome of their inability to perceive or experience the pain that they feel as a threat, danger, or damage to the integrity of their body and mind. However, this pain which does not represent threat, danger, or damage for asymbolia patients and in no way alarms them, but makes them only smile or laugh, poses gross threat both to the subjectivist and to the objectivist conceptions or interpretations of the true nature and structure of human pain experience. Actually, by quite unexpected ironical twist, it threatens to inflict an irreparable theoretical damage to these conceptions of pain or at least threatens to stay a permanent pain in the neck for them. In order to substantiate this pretty grim and pessimistic claim, I will first consider how the case of pain asymbolia might affect the viability of the subjectivist conception of pain, and will then proceed to examine the damaging impact that it may have on the objectivist interpretation of pain experience.

Now, according to the subjectivist view, the sensation of pain with its distinctive phenomenal content or quality - the what-it-is-likeness of pain - is the essential component of our total pain experience and plays the central or fundamental role in it. When this component is absent, there is no pain or pain becomes ersatz pain, despite the presence of all other components of pain experience; and this is something that standard absent qualia argument is purported to conclusively prove. If, according to subjectivists, the sensation with pain quality really plays this essential or central role in pain experience, it seems that they would be committed to claim that its presence is sufficient for somebody to be in pain. Actually, they might be tempted or even forced to claim that, in the case of pain asymbolia, where this components is present, while all other components of pain experience are conspicuously absent, we have finally come to the very essence of pain; that the pure juice of pain quality - the what-it-is-likeness of pain - has been extracted and clearly presented. But, to the disappointment of many, myself very much included, the pure juice or essence of pain experience thus extracted, has turned out to be a blunt, fleshless, inert sensation pointing to nothing beyond itself, leaving no traces in the memory and powerless to move the body and mind in 
any way. Moreover, when reduced to pure sensation, pain becomes the object of ridicule. In other words, the legendary question what it is like to be in such pain, would get the following answer: it is the pain that makes one smile and laugh! But then one would strongly be inclined to say that this is not real pain; that it is only mock pain. By ironical twist, the case of pain asymbolia would show that what is supposed to be the essence of pain actually puts one into the state of ersatz pain. To put this point into other terms, the case of pain asymbolia would quite unexpectedly prove that, contrary to subjectivist's claims, the sensation of pain is not the essential component of our total pain experience; that it does not play any central or fundamental role in it. It could be used as an ironical sting against the following subjectivist's memento put forward by Campbell in highly rhetorical biblical terms: "To a considerable extent, so far as many mental states go, by their qualia shall ye know them. To an even greater extent, by their qualia shall ye value them, imagine them, remember them, and fear them" (Campbell, 1983, p. 136). As the case of pain asymbolia vividly and conclusively shows, when pain is reduced to pure quale it looses any force that would make one remember it, have a fear of it, or esteem it as a threat or danger.

Actually, this most peculiar and bizarre reactive dissociation syndrome to be found in human pain experience, where only the sensation of pain is present, would strongly speak in favor of Wittgensteinian beetle-in-the-box argument purported to prove that the quality of the sensations that one feels is quite irrelevant for determining whether somebody is in pain. In other words, what is in the box does not matter for pain: what does matter is what one believes, how one feels (affectively not sensorially) and acts (Wittgenstein, § 293, 1968). Of course, the case of pain asymbolia or the pain that one finally smiles or laughs at would certainly be, as far as the crucial components of human pain experience are concerned, the best possible evidence that Wittgenstein was right when he has remarked that "a wheel that can be turned though nothing else moves with it, is not part of the mechanism" $(1968, \S 271)$. It would also strongly speak in favor of Valerie Hardcastle's intention to show, in her book The Myth of Pain, "that the sensation of pain - what most philosophers of mind 
focus upon as absolutely central to being in pain - is neither a particularly fundamental nor a particularly important component to our pain processing ... for what something is like becomes less important in explaining our mind" (1999, p. 94). However, we will have to examine more carefully whether the case of pain asymbolia would really and fully justify this resolute and conclusive verdict on the role of the sensation of pain in our total pain experience. But before we do that, we have to consider more closely how the case of pain asymbolia is supposed to affect the tenability or plausibility of the objectivist conception of the true nature and structure of pain. This conception of pain is to be found in the so-called perceptual or representational model of pain that was devised by naturalistically minded philosophers in order to show that, contrary to subjectivist's claims, there is no intrinsic and irreducible felt quality or phenomenal content in pain experience; that the phenomenal content of pain can be, without remainder, analyzed in purely representational or relational terms. According to this model, the feeling of pain is to be understood just as the awareness of objective bodily state of affairs: as the perception or sensory representation of bodily or tissue damage. Besides, pain sensory system is to be conceived as any other perceptual system: for instance, visual or tactual, the only difference being that it represents some internal, bodily changes, while the latter are directed at objects in the outside world.

The first fully elaborated and explicitly stated perceptual model of pain was put forward by Pitcher: "to be aware of pain is to perceive in particular, to feel, by means of the stimulation of one's pain receptors and nerves - a part of one's body that is in the damaged, bruised, irritated, or pathological state, or that is in the state that is dangerously close to being one or more of these kinds of states" (1970, p. 371). The most recent version of the fully objectivist conception of pain is to be found in the writings of $\mathrm{M}$. Tye, the only difference being that it now goes under the heading of the representational model or theory of pain. This model of pain is actually elaborated within the more general representationalist model that is supposed to apply to all experiences and feelings: 
The picture that emerges from my discussion is one of the experiences and feelings as sensory representations either of the outside world or of certain sorts of internal, bodily changes. Moods, emotions, and bodily sensations, in my view, are importantly like maps of our own internal physical workings, guides to our inner body states, graphic representations of what is going on inside (and to) our skins. Perceptual experiences are representations of the same sort, but their focus is the outside world, the external terrain. (Tye, 1995, p. 94)

When this general picture is applied to pain experience, we get the following representational model of pain:

My proposal, then, is that pains are sensory representations of bodily damage or disorder. More fully, they are mechanical responses to the relevant bodily changes in the same way that basic visual sensations are mechanical responses to proximate visual stimuli. In the case of pain, the receptors (known as nociceptors) are distributed throughout the body. These receptors function analogously to the receptors on the retina. They are transducers. They are sensitive only to certain changes in the tissue to which they are directly connected (typically, damage), and they convert that input immediately into symbols. Representations are then built up mechanically of internal bodily changes, just as representations are built up of external surfaces in the case of vision. These representations, to repeat, are sensory. They involve no concepts. One does not need to be able to conceptualize a given bodily disturbance in order to feel pain. And even if one can, it is not relevant, because feeling pain demands the sensory experience of that disturbance. (Tye, 1995, pp. 113-114)

That the feeling of pain is not, by itself, the perception or representation of bodily or tissue damage, as it is claimed by the perceptual or representational model of pain is, indeed, quite well and convincingly documented by the case of pain asymbolia patients. Namely, as the thoroughly and repeatedly conducted neurological and psychophysical examinations of these patients have shown, they are quite capable 
to discriminate, differentiate, and localize the damaging or potentially damaging stimuli whenever they are applied at any part of their bodies, and they do feel pain on that account. However, the pain that these patients regularly feel on such occasions does not at all represents for them the damage or potential damage to parts of their bodies. And that this is so is best proved by the fact that they consistently smile or laugh during pain testing procedures. If that is the case, one can safely claim that the sensation of pain does not carry, by itself, any representational force; that, when present alone, it comes to nothing in the sense that it in no way carries the "meaning" of physical damage or at least threat to the physical well-being. So, contrary to the perceptual or representational model of pain, the feeling of pain cannot, when taken alone, be understood as the perception or representation of the bodily or tissue damage. In other words, it seems that the representational force of pain is rather to be sought in the emotional-cognitive components of pain. As Chapman and Nakamura have remarked:

The strength of emotional arousal associated with an injury indicates, and expresses, the magnitude of perceived threat to the biological integrity of the individual. Within the content of consciousness, threat is realization of a strong negative feeling state and not a coldly calculated informational appraisal. The emotional magnitude of a pain is the internal representation of the threat associated with the event that produced pain. (1999, p. 400)

But this is something that has much earlier been realized by Sherrington:

With its liability to various kinds of mechanical and other damage in a world beset with dangers amid which the individual and species have to win their way in the struggle for existence we may regard nocuous stimuli as part of a normal state of affairs. It does not seem improbable, therefore, that there should under selective adaptation attach to the skin a so-to-say specific sense of its own injuries. As psychical adjunct to the reactions of that apparatus we find a strong displeasurable affective 
quality on the sensations they evoke. This may perhaps be a means for branding upon memory ... a feeling from past events that have been perilously critical for the existence of the individuals of the species. In other words, if we admit that damage to such an exposed sentient organ as the skin must in the evolutionary history of animal life have been sufficiently frequent in relation to its importance, then the existence of a specific set of nerves for skin-pain seems to offer no genetic difficulty, anymore than does the clotting of blood or innate immunity to certain diseases. (1948, pp. 228-229)

Sherrington has obviously recognized that pain is a sensory sub-modality subserved by the physiologically specialized nociceptive neural system whose function is to detect harmful or potentially harmful stimuli and transmit the sensory information about these stimuli to higher cortical areas for final processing of their properties and significance. Actually, without adequate electrophyisiological and psychophysical evidence, he has anticipated the existence of nociceptive neurons that preferentially or distinctively respond to noxious or potentially noxious stimuli, and has coined the very term for this physiologically specialized class of neurons (Sherrington, 1948, p. 229). That is why he speaks that attached to the skin there is a specific sense of its own injuries. But he has also realized that pain is not a simple sensory registration of noxious or potentially noxious stimuli. That is why he speaks about the strong displeasurable affective quality in the sensations that the activity of the nociceptive apparatus evokes. Namely, it is this component of pain that leaves traces in the memory about the threatening, dangerous, or damaging stimuli and makes an organism learn to protect itself by avoidance or defense behavior from such stimuli. And this is exactly something that is conspicuously missing in pain asymbolia patients, despite the fact that they are quite capable of the sensory registration of noxious or potentially noxious stimuli by feeling pain at the part of their body that has been injured and discriminating its quality as sharp, dull or burning. So, to put the main point once again, although these patients feel pain, it simply doesn't represent for them the sign of threat, danger, or damage to their bodies: it is, for them, a mock threat that they smile or 
laugh at. Now, the perceptual or representational model of pain is grounded on the experiential, physiological, and psychophysical evidence that pain is, indeed, the sensory registration of damaging or potentially damaging stimuli subserved by the specialized nociceptive neural apparatus. That much is true. But the sensory registration of body or tissue injury or the sensation of pain felt at the site of such injury do not, by themselves, represent for the subject the physical damage to his body or at least the threat to his physical well-being. That much is clear from the consideration of the case of pain asymbolia. In other words, nociceptive signals may evoke the sensation of pain for sensory discrimination of harmful or potentially harmful stimuli, but they need to be processed further for their affective and motivational valence as well as for their behavioral significance, if pain is to represent or "mean" for an organism that physical damage has been inflicted or that there is a at least a threat to its well-being. And that is the truth of the matter as far as human pain experience is concerned.

The consideration of the case of pain asymbolia or the pain that doesn't carry any representational and motivational force, has shown that the major fault of the perceptual or representational view of pain is to be found in the fact that it failed to capture the real mode in which pain can come at all to represent for the subject the threat or damage to his body; that it wrongly located this basic, primitive representational capacity of pain in the sensory-discriminative aspect of pain experience, instead of looking for it in its emotional-cognitive dimensions. And closely related to this mistake is the erroneous claim, made by the advocates of the perceptual model of pain, that pain sensory system differs from visual or tactual systems only with regard to the objects that they are directed at or which they are supposed to represent. For to make such a claim means to disregard the fact that the former sensory system, unlike the latter sensory systems, is also inherently endowed with characteristic affect that motivates behavior: that all sensations "referred to the body itself, rather than interpreted as qualities of objects in the external world, tend to be 'tinged' with feeling" and that "sense-organs which initiate sensations 
tinged with feeling tend to excite motor centers directly and imperatively" (Sherrington, 1948, p. 267).

The failure of the perceptual or representational model of pain to capture and account for the representational force of pain reminds one of the failure of the project devised to build an artificial pain system. The project that went under the heading, "A Practical Substitute for Pain", was devised and conducted by Paul Brand in order to replace the defective system in people who suffered from leprosy, congenital painlessness, diabetic neuropathy, and other nerve disorders. In a nutshell, the project had the following major goal:

We planned, in effect, to duplicate the human nervous system on a very small scale. We would need a substitute "nerve sensor" to generate signals at the extremity, a "nerve axon" or wiring system to convey the warning message, and a response device to inform the brain of the danger.

One of the engineers ... joked about the potential for profit: "If our idea works, we'll have a pain system that warns of danger but doesn't hurt. In other words, we'll have the good parts of pain without the bad!" (Brand and Yancey, 1997, p. 192)

A team of electrical engineers developed a miniature sensor for measuring temperature and pressure: that is, transducers that were slim metal disks smaller than a shirt button. Sufficient pressure on these transducers would alter their electrical resistance, triggering an electrical current. The first problem was to determine what thresholds of pressure and temperature should be programmed into the sensors:

After many compromises we settled on baseline pressure and temperatures to activate the sensors, and then designed a glove and a sock to incorporate several transducers. At last we could test our substitute pain system on actual patients. Now we ran into mechanical problems. The sensors, state-of-the-art electronic miniatures, tended to deteriorate from metal fatigue or corrosion after a few hundred uses. ... Worse, the sensors cost about $\$ 450$ each and leprosy patient who took a long walk around the hospital grounds could wear out a $\$ 2,000$ sock! (Brand and Yancey, 1997, pp. 193-194) 
But the point is that there were even more serious problems related to the very efficacy or successful functioning of the artificial substitute pain system:

Even when the transducers worked correctly, the entire system was contingent on the free will of the patients. We had grandly talked of retaining "the good parts of pain without the bad", which meant designing a warning system that would not hurt. First we tried a device like a hearing aid that would hum when the sensors were receiving normal pressures, buzz when they were in slight danger, and emit a piercing sound when they perceived an actual damage. But when a patient with a damaged hand turned a screwdriver too hard, and the loud warning signal went off, he would simply override it - This glove is always sending out false signals - and turn the screwdriver anyway. Blinking lights failed for the same reason. (Brand and Yancey, 1997, p. 194)

So here we have the substitute pain system that is in full accord with Tye's representational model of pain and allegedly provides an organism or subject with sensory representations of bodily damage or disorder. There are specialized transducers sensitive to damaging or potentially damaging stimuli and they convert these inputs directly into symbols, so that representations are then built up mechanically of "tissue" or "bodily" changes. And these representations are, indeed, sensory, as Tye is eager to repeat: a piercing sound is emitted when an actual danger is detected and the quality of this acoustic experience phenomenally corresponds to the quality of piercing pain. But the substitute pain system failed in its major substitutive function: to alarm the patient about the damaging stimuli and make him avoid them. What went wrong? Well, the substitute pain system failed for much the same reasons that the representational model of pain fails to capture the real representational force of pain. The signals or messages that the patient was receiving simply didn't represent to him the physical damage or at least threat to the physical well-being. They were for him false signals which he just ignored, and this fact reminds one very much of the peculiar attitude that pain asymbolia patients have toward the very sensation of pain. Namely, for these patients the 
very pain that they feel upon harmful stimulation at a particular site of their body is also a false alarm which they ignore or even smile or laugh at. For we have only to remind ourselves that the wife of one of these patients reported that he had accidentally suffered a serious left hand burn without escape or emotional reactions. In order to rescue the substitute pain system from total ignorance and eventual ridicule, the team working on the project "A Practical Substitute for Pain" had to improve or upgrade the system, so that it could properly and efficiently perform its basic function:

The sobering realization dawned on us that unless we built in a quality of compulsion, our substitute system would never work. ... Professor Tims of LSU said to me, almost in despair, "Paul, it's no use. We'll never be able to protect these limbs unless the signal really hurts."

We tried every alternative before resorting to pain, and finally concluded Tims was right: the stimulus had to be unpleasant, just as pain is unpleasant. One of Tims's graduate students developed a small battery-operated coil that, when activated, sent out an electric shock at high voltage but low current. It was harmless but painful, at least when applied to parts of the body that could feel pain.

Leprosy bacilli, favoring the cooler parts of the body, usually left warm regions such as the armpit undisturbed, and so we began taping the electric coil to patients' armpits for our tests. ... I noticed though, that they viewed pain from our artificial sensors in a different way than pain from natural sources. They tended to see the electrical shocks as punishments for breaking rules, not as messages from an endangered body part. They responded with resentment, not an instinct of self-preservation, because our artificial system had no innate link to their sense of self. How could it, when they felt a jolt in the armpit for something happening to the hand?

I learned a fundamental distinction: a person who never feels pain is task-oriented, whereas a person who has as an intact pain system is self-oriented. The painless person may know by 
a signal that a certain action is harmful, but if he really wants to, he does it anyway. (Brand and Yancey, 1997, pp. 194-195)

One could say that the failure of this improved or upgraded artificial substitute pain system is even more striking, because this time the very pain - to put the matter in somewhat paradoxical but quite true terms - was used to serve as part of the substitute pain device. Tye could claim that this spectacular failure was to be expected for the simple reason that the pain elicited by the activity of artificial nociceptive sensors positioned on the hand was felt as located in the armpit. In other words, the pain was not the sensory representation of damage to the body part that was actually exposed to harmful stimulus - the hand - and thus the patient couldn't internalize it as the sign of threat or danger to that part of his body. Tye would certainly be right on this point, and this is something that Brand himself has noticed and offered as one of the explanations for the fiasco of his "painfully" upgraded substitute pain project. However, this substitute pain system could be, in principle, upgraded one step further so that the mismatch between the location of the sensation felt and the site of the body damage would be avoided. For this purpose, signals coming from the "hand" artificial nociceptive sensors and transducers in leprosy patient should be transmitted to electrodes inserted into the primary and secondary somatosensory areas of the brain, so that their activity would evoke the response of the somatotopically organized nociceptive neurons whose receptive fields are in the hand. In this way the sensation of pain evoked by the damaging mechanical or thermal stimulus applied to the hand would be projected to that body part: that is, pain would be felt as located in the hand.

Would that solve all the problems for the construction of the fully effective substitute pain system? Would the pain, now projected or felt as located at the body part that is actually suffering damage, represent for the leprosy patient the threat or danger for the integrity of that particular part of his body? Yes, if the signals coming from "hand" artificial nociceptive sensors and transducers, were further transmitted to the cortical areas responsible for the processing of their affective and motor valence as well as their behavioral significance. No, if there would be no such further processing. Unfortunately, even 
if we had the completely "painful substitute pain system" it would still not function as the real pain system The major deficiency of such, almost perfect semi-artificial pain system, would consist in the fatal weakness that Brand has found in his initial project:

Most important, we found no way around the fundamental weakness in our system: it remained under the patient's control. If the patient did not want to heed the warning from our sensors, he could always find a way to bypass the whole system. Looking back, I can point to a single instant when I knew for certain that the substitute pain project would not succeed. I was looking for a tool in the manual art workshop when Charles, one of our volunteer patients, came to replace a gasket on a motorcycle engine. ... One of the engine bolts had apparently rusted, and Charles made several attempts to loosen it with a wrench. It did not give. I saw him put some force behind the wrench and then stop abruptly, jerking backward. The electric coil must have jolted him. ... Charles studied the situation for a moment, then reached up under his armpit and disconnected a wire. He forced the bolt loose with a big wrench, put his hand in his shirt again, and reconnected a wire. It was then that I knew we had failed. Any system that allowed our patients freedom of choice was doomed. (1997, pp. 195-196).

It is obvious that our imagined perfectly "painful substitute pain system" would be doomed for the same reason: the patient would at any time be able to disconnect the wire and proceed with actions that can inflict serious injuries to his body. This would certainly be irrational, but there would be nothing to stop these irrational acts: "The mysterious power of the human brain can force a person to STOP - something I could never accomplish with my substitute system" (Brand and Yancey, 1997, p. 196). On the other hand, if the leprosy patients were equipped with the substitute mechanism that projected pain sensations to the proper body part, but was short of painfulness, they would not be tempted at all to disconnect a wire and would continue with self-injurious behavior. The insuperable "disconnection or unplug problem" that the substitute pain system faces would be miraculously solved, but at the price that the pain felt as located at the 
real site of the injury would not represent for leprosy patient any danger or threat, and would not make them avoid the damaging or potentially damaging stimuli. In other words, they would not be tempted to disconnect a wire and bypass the whole substitute pain system for the same reason for which asymbolia patients do not attach any significance to the pain that they feel upon harmful stimulation or even smile or laugh at it. The simple reason being that in both cases the pain would be accurately located, qualitatively discriminated, properly temporally registered and calibrated for its intensity, but would come to blunt, inert sensation pointing to nothing beyond itself and in no way moving the mind and body. So, in these cases there would be no problem related to the spatial mismatch between the site at which sensation is felt and the site of the injury, but there would still be left some quite serious and deep problems. And these problems are actually related to the fundamental shortcomings of the representational model of pain: to the fact that this model is basically deficient as the conceptual model of pain and that it implicitly presupposes a too simplistic and inadequate picture of the neural mechanism that is supposed to subserve human pain experience.

The representational model of pain is essentially grounded on the idea of simple sensory registration of pain as a message. The message is unpacked as the sensory representation of bodily damage or disorder or is supposed to carry, in strictly sensory terms, information about such damage or disorder. The neural mechanism of pain presupposed by this model is the one given by classical neurophysiology: that of nociception, noxious signaling and sensory registration of pain as predominantly bottom-up, unidirectional, sequential type of processing. But, as the consideration of the case of pain asymbolia has convincingly shown, the sensation of pain does not carry by itself any message or representation of the bodily damage, if nociceptive signals are not further or in parallel processed for their affective and motor valence as well as for their behavioral significance. The classical sensory neurophysiological view of pain, as well as the representational model of pain which is grounded on it, are faced with the following theoretical problems or perplexities: 
This position has major problems in explaining how a sensory experience can contribute so powerfully to suffering: why pain "hurts" is still unclear. Also, this approach cannot address the knotty problem that challenges consciousness researchers how do signals of tissue trauma make their way into consciousness? (Chapman et al., 2000, p. 28)

An important part of the problems or puzzles mentioned above can be dispelled if the more complex and veridical picture of the neural mechanism that subserves human pain experience is taken into account. As we have already said, the representational model of pain, as well as the classical sensory neurophysiological picture of pain on which it is grounded, have every right to claim that pain is subserved by the physiologically specialized nociceptive neural system whose function is to detect harmful or potentially harmful stimuli and to transmit the sensory information about these stimuli to higher cortical areas for final processing of their properties and significance. However, the information about the noxious or potentially noxious stimuli, or at least the one related to their intensity, is at the thalamic level transmitted through parallel routes simultaneously activating various spatially separate cortical and subcortical areas responsible for feature-extraction, affective evaluation, attention attraction, and motor processing of the incoming nociceptive signals. Coghill and his research team have recently conducted a detailed and precise imaging study in order to determine which cortical and subcortical regions of the brain are involved in the processing of pain intensity: that crucial factor for the assessment of the danger or threat that noxious or potentially noxious stimuli carry for the integrity of the body and mind. The results of this study are summarized in the following general way:

Multiple regression analysis of the functional imaging data revealed that a number of cerebral cortical and subcortical areas exhibited significant, graded changes in activation linearly related to subjects' perceptions of pain intensity. The findings from the multiple regression analysis are independently supported by comparisons of PET scans at each stimulus temperature with the resting state. In most areas, innocuous $\left(35^{\circ} \mathrm{C}\right)$ and 
threshold $\left(46^{\circ} \mathrm{C}\right)$ stimulation produced minimal differences from rest. However, as stimulus temperature increased to $48^{\circ}$ and $50^{\circ} \mathrm{C}$, monotonic increases in activation were evident in multiple brain areas. (Coghill et al., 1999, p. 1936)

In the discussion of the obtained results, the basic findings are presented in more specific and precise manner, and important implications for our understanding of the neural processing of pain intensity are drawn:

The present findings confirm in a fully quantitative manner that pain intensity is processed in a highly distributed manner. This distributed mechanism encompasses a number of functionally distinct regions that all exhibit activation that is closely related to perceived stimulus intensity. These include brain areas typically thought to be important in 1) somatosensory processing: SI, SII, and the posterior insular cortex; 2) motor processing: cerebellum, putamen/globus pallidus, supplementary motor cortex, ventral premotor cortex, and the anterior cingulate cortex; 3) affective processing: anterior cingulate cortex and insular cortex; 4) attentional processing: anterior cingulate cortex, primary somatosensory cortex, and the ventral premotor cortex; 5) autonomic function: anterior cingulate cortex and anterior insular cortex.

Multiple, converging lines of evidence indicate that this distributed processing of pain intensity information rests on a parallel infrastructure of nociceptive transmission. First, anatomic evidence indicates that information about noxious stimulus intensity may be transmitted independently from thalamic sites to cerebral cortical areas such as SI, SII, the insular cortex, the anterior cingulate cortex, the supplementary motor cortex, and the ventral premotor cortex. Second, neurological evidence confirms that these multiple thalamocortical pathways are functionally relevant. Discrete injuries of either SI, SII, anterior cingulate cortex, or the insula fail to abolish conscious awareness of pain intensity, although other aspects of processing may be disrupted somewhere. Thus serial transmission of nocicep- 
tive information through any one of these cerebral cortical areas is not obligatory for a conscious awareness of the intensity of painful stimulus. (Coghill et al., 1999, pp. 1939-1940)

The picture of the pain neural mechanism that works as a parallel distributed mechanism gives us a clue how to approach the problems or puzzles that seems to be completely intractable within the main premises of the representational model of pain. How sensory experience can contribute to suffering? Why pain "hurts"? How can the sensory representation, which is and should be hedonically neutral, give rise to dislike and strong aversive reactions? How do signals of tissue trauma make their way into consciousness? Once we realize that nociceptive signals about damaging or potentially damaging stimuli are in parallel transmitted for simultaneous sensory-discriminative and emotional-cognitive processing, the mystery is dispelled, because the signals are simultaneously processed for their painfulness and their pain character. To put this point into more precise terms, the aversive valence and biological significance of nociceptive signals is processed in parallel and simultaneously with the processing of the location at which the pain sensation is to be projected and its intensity and qualitative character decided. That the mechanism is working as a parallel distributed mechanism, and not as the serial one, is proved by the cases of pain asymbolia and pain affect without pain sensation. The first case shows that the registration and the processing of sensory features of nociceptive signals can go on and can be successfully accomplished without any processing of their aversive valence and biological significance. The second case shows that nociceptive signals can be registered and processed for their aversive valence and biological significance without any processing of their sensory features. Of course, except in these two cases of radical disconnections between the cortical and subcortical areas of the brain responsible for sensory-discriminative and emotional-cognitive dimensions of pain, they are working reciprocally and interactively, rather than independently. That is the reason why, phenomenologically, they appear to us in homogenous, gestalt guise, as they really should, if pain is to serve its major purpose to alarm an organism with regard to damaging or potentially damaging stimuli and determine the direction and intensity 
of responses; if it is to have, to use Sherrington's terminology, preeminent intensity of feeling and, consequently, of action. So, the following remark, made by Millan, is highly justified and should always be kept in mind:

Nevertheless, it would be simplistic to impose an absolute dissociation between the sensory-discriminative and emotionalcognitive dimensions of pain. These aspects should be regarded as complementary and as operating reciprocally and interactively rather than independently. Further, a 'pain center' or 'pain centers' may not, as such, exist. Rather, a matrix of cerebral structures and multiple, parallel thalamocorticolimbic networks synergistically contributes to the global experience of pain. (Millan, 1999, p. 40) 


\section{Pain Quality and Painfulness Without Pain}

As I have already said, the fact that for asymbolia patients the pain that they feel comes to nothing or to something that they smile or laugh at, may seem, at first sight, to lend full support to the radical anti-subjectivist claim that the sensation of pain or pain quality plays no important role in our total pain experience: what really and only matters is how we respond affectively, what we believe, and how we act. But as Valerie Hardcastle herself has quite rightly warned philosophers, dissociation syndromes in human pain experience should not be used or abused in order to conclusively show that only one of its components or dimensions is central or essential for pain (1999, p. 94). And this warning should certainly be taken seriously in the case of pain asymbolia: that most radical reactive dissociation syndrome to be found in human pain experience. Namely, the only conclusion that one is really allowed to draw from the consideration of this syndrome is that pain sensation comes to nothing when it is disconnected from the relevant affective, cognitive, and motor machinery. There is nothing in this conclusion that would suggest that it does not play any role in pain experience or that even in its complete absence that experience would still be pain experience. It is obviously the triggering point which sets the machinery to go, and it sets apart the machinery that wears its sign from those that are similar or partially similar to it, but do not carry its signature. In other words, the role of the sensation with distinctive pain quality is, first, to distinguish sensory pain from the so-called mental pain or suffering; second, to set it apart from other unpleasant sensations by its distinctive quality; third, to differentiate it from other, phenomenally similar sensations, and give unity to the sensations that pertain to the sensory modality of pain.

As far as the first role is concerned - that of distinguishing sensory pain from non-physical pain or mental hurting - it is sufficient to quote at the beginning the following remark made by Sherrington: "The disagreeableness of a vivid color contrast or of discordant notes is akin to pain but is it really a degree of 'physical pain'? Is it strictly expressible as a fraction of the agonizing torture of a scalded limb?" (1900, p. 968). To make this point even more vivid and convincing, 
Sherrington himself quotes with complete approval Foster's observation that "the pain which we feel when the finger is cut is a wholly different thing from the pain given to the most delicately musical ear by even the most horrible discord" (1900, p. 968). Now, the point of this comparison was to show that in the first case there is a sensory quality present which is missing in the second case; and that it is exactly the absence of this quality which differentiates the pain of listening to the badly played music from the pain felt when the finger is cut, however much both of them may be unpleasant. And this brings us to the consideration of the second important role that sensation with distinctive pain quality plays in our total pain experience: namely, the role of setting apart pain from other unpleasant sensations from which it can be only distinguished by its sensory quality or modality. Nausea, for one, is such a case; but as Sherrington has quire rightly observed, "some sensations of taste, e.g. bitter, sour, certain odors, possess an unpleasant character - a 'negative tone' - almost at the limen of the sensible intensity of the stimulus. Here the disagreeableness seems to be allied, not to the intensity, but to the 'timbre' or 'color' or quality of the sensation" (1900, p. 967). As far as the experimental confirmation of this claim is concerned, one should mention that the experiments conducted by Henry Head have led him to make the distinction between the sensory quality of pain and the feeling-tone of discomfort which usually accompanied it. He especially examined the effect of an electric current on a leg which was "totally insensitive to painful stimulation of all kinds, in consequence of an intramedullary lesion"; and he has found that "so long as tactile sensibility remains perfect (the patient) will complain bitterly of the discomfort caused by this form of stimulation" (1920, p. 405). Experiments with two patients "showed that the movement of withdrawal seemed to be almost as violent when a current of known strength was applied to the analgesic as to the normal leg"; and one patient said that the sensation produced was "a kind of exaggerated tickling more unpleasant than pain" (Head, 1920, p. 405). Both patients were firm in their assertions that the sensation was not painful; and yet an observer watching their behavior would suppose they were undergoing intolerable pain. As Roger Trigg has observed, situations 
like these "seem to show that the emotional component of pain can be combined with some types of sensations and yet neither the resulting complex nor the 'bare sensation' (if such thing is possible) be called "pain"' (1970, p. 23). As Ryle in his book The Concept of Mind has observed: "It should be mentioned that 'pain', in the sense in which I have pains in my stomach, is not the opposite of 'pleasure'. In this sense, a pain is a sensation of special sort, which we ordinarily dislike having" (1973, p. 105).

Pain is, indeed, a sensation of special sort; the sensory sub-modality or experiential determinable that gives unity to sensations that carry its mark, and sets them apart from sensations that, at the level of experiential determinates, may be qualitatively similar to them, but are of different modality. Namely, the pain that one feels at a certain part of one's body upon mechanical or thermal stimulation can be dull or sharp, stinging or burning. That is, the sharp or dull as well as the stinging or burning qualitative character of the sensation felt - of what one's pain feels like - may correspond to the qualitative differences within the modality of pain, designating different kinds of pain within the same genus. Or, to put this point into other terms, sharp pain and dull pain as well as stinging pain and burning pain are experiential determinates of the experiential determinable the sensation of pain. But sensations that are felt as sharp or dull, as well as those that are felt as stinging or burning, may also correspond to the intra-modal differences within the modality of the sensation of touch or the sensation of heat. That means that one can discriminate the mechanical or thermal stimuli as sharp or dull or as stinging or burning and yet not feel pain, because the sensations felt do not carry pain quality. In order to be felt as painful, these sensations have to pertain to the modality of pain; otherwise, the subject will not feel pain despite the fact that he feels sensations that he discriminates as sharp or dull or as stinging or burning. So it seems that the concept of pain quality or the concept of pain modality really plays the important role of something that gives unity to sensations that go under the heading of pain and sets them apart from those that are qualitatively similar but of different modality: of something that serves to embrace them so as to mark their territory and boundaries. 
That this is so may be vividly illustrated and clearly documented by the consideration of clinical syndromes that are in most behavioral and affective respects similar to pain asymbolia syndrome, but differ from it in one crucial or decisive phenomenal respect: namely, in the obvious absence of the very feeling of pain which is so conspicuously present in the latter syndrome. The syndromes that I have in mind are the cases of the so-called congenital insensitivity to pain or, better, congenital analgesia and the cases of acquired analgesia due to brain trauma. Now, the interesting thing about these syndromes is the fact that analgesia - loss of sensitivity to pain - is not always followed by anesthesia - total loss of sensation in all or part of the body. Namely, patients suffering from congenital analgesia similarly to pain asymbolia patients do not show common reactions to noxious or potentially noxious stimuli:

The examination of pain reaction was done in many ways. All the usual noxious stimuli - pricking with a pin, electric shocks, pressure on sensitive parts, hair-pulling, burning, and pinching - were used. Other tests included ... tests for the production of muscle pain, the production of "cold pain" from immersion of the hand in cold water ... and the production of headache by injection of histamine phosphate.

Absence of reaction in nearly all these tests means that the patients did not show the usual behavior of withdrawal, attack, wincing, crying out, or reporting pain. ... Also, the most striking evidence of insensitivity to pain rests usually in the patient's past history of indifference to everyday noxious stimuli, many of them much more damaging than could be administered for purposes of examination. (McMurray, 1955, pp. 124-125)

Like pain asymbolia patients, those who suffer from congenital analgesia are in some cases not actually insensitive to noxious stimulation:

... they can detect, identify, and localize noxious stimuli and can easily differentiate them from other stimuli. McMurray's ... $S$ states that, when a hypodermic needle is inserted into her skin, she feels it penetrating the tissue layers but does not "feel 
pain". Stimuli such as pinprick and cutaneous shock and heat produce the report of a pricking or sharp quality, but she does not describe this quality as "painful". In fact, since this $S$ can discriminate the sharp quality of heat stimulation, McMurray was able to establish in the patient a "threshold" close to the normal heat pain threshold. Similarly, (other authors ...) have reported that their $S$ s had no difficulty differentiating and localizing a nociceptive stimulus; they could, for example, easily discriminate between the blunt and pointed end of a pin and had no difficulty localizing the pinprick. (Barber, 1959, p. 443)

So it seems that the major difference between pain asymbolia patients and some patients suffering from congenital analgesia is exactly to be found in the fact that only former feel pain on account of noxious stimulation; that only they have sensations with pain quality on such occasions. Patients suffering from the latter syndrome can discriminate the sharp, pricking qualitative character of correspondent mechanical or thermal noxious stimuli. But they do not feel pain, because the sensations that they feel do not carry pain quality or simply are not sensations of pain. And that again shows that the role of pain quality or the concept of separate pain modality is to unite and distinguish the class of sensations that go under the heading or genus of pain. But the concept of pain quality or the sensation of pain is also needed for diagnostic purposes: namely, to make distinctions between clinical syndromes that may be, as far as pain experience is concerned, similar with regard to the absence of appropriate behavioral and affective reactions to painful stimuli but are, nevertheless, phenomenologically different with respect to the modality of sensations felt on such occasions. As Trelles has observed, pain asymbolia can be understood "as analgotimia with algognosia while congenital analgesia ... is at the same time a case of analgotimia with analgognosia" (Trelles, 1978, p. 18). That is to say, in the first case one is not in pain but feels pain while in the second case one is neither in pain nor feels pain.

The importance of the distinction between feeling pain and being in pain for clinical diagnostic purposes can also be seen in the case of the patient studied by Masson et al. Namely, due to the trauma to the 
posterior insula, the parietal operculum and the supramarginal gyrus, this patient had on the left side of the body severe hemianesthesia or complete loss of sensation: "The most spectacular deficiency was related to pain sensibility which was completely lost: pinprick was not perceived and the same holds for vigorous nociceptive stimulations ... the patient denied all painful perception, made no movements of escape and had no appropriate grimacing at all" (Masson et al., 1991, p. 668). However, on the right side of the body, "the patient was perfectly able to discriminate the touch of the needle, but the application of strong nociceptive stimulation didn't evoke any painful sensation and didn't elicit any reaction of escape. The patient who was warned about the painful character of the stimulations that are going to be applied manifested no emotional reaction" (Masson et al., 1991, p. 670). Masson et al. are inclined to treat the case of their patient as the case of pain asymbolia. But it is quite obvious that their patient doesn't comply to the stringent diagnostic criteria for pain asymbolia put forward by Berthier, Starkstein, and Leigurda. Namely, pain asymbolia patients have no deficit in pain perception on either side of their body, although they have absent or inadequate motor and emotional responses to painful stimuli applied anywhere on the body surface. Consequently, the case of the patient studied by Masson et al. should actually be treated as the case of acquired severe hemianesthesia on the left side of the body and hemianalgesia on the right side of the body. The case of this patient as well as the cases of patients suffering from congenital analgesia not followed by anesthesia are, in many respects, observationally indistinguishable from the cases of pain asymbolia patients. Namely, for all these cases is characteristic the absence of adequate motor and emotional reactions to harmful stimuli. Besides, this common deficit can lead to common disastrous consequences as far as the integrity and well-being of patient's bodies is concerned. For we have to remember that pain asymbolia patients, as much as patients with congenital analgesia, may suffer serious injuries without escape or emotional reactions. However, there is a crucial and decisive phenomenological difference between these patients, which is to be found in the fact that only former feel pain on such occasions: that is, both groups of patients are not in pain, but 
only patients of the first group have sensations with pain quality. So it seems that the distinction between feeling pain and being in pain is viable, and that the concept of pain quality is indispensable to give unity to the sensations that carry its stamp and set them apart form those that may be similar to them, but are of different modality.

In his famous dialogue Meno, Plato has remarked that the major task of science (philosophy) is to cut nature at its joints: that is, to make distinctions that correspond to the real distinctions in the world; or, to put the same point into different words, not to lump together phenomena that are of different nature and that, accordingly, need to be given different descriptions and explanations. As the same holds for the world of mental phenomena, we should not lump together clinical syndromes that may be observationally almost indistinguishable, but nonetheless differ in one critical or fundamental phenomenological respect: that is, in the absence or presence of the very sensation of pain. At this point verificationists or anti-realists - both of parochial and urbane variety - will vigorously protest in order to defend their basic epistemological and metaphysical convictions, and will resolutely claim that differences that make no observational difference should be absolutely dismissed or discounted. Now, there might be something in this epistemologically and metaphysically inspired request, but the point is that there is also an important and decisive difference in the behavioral pattern between pain asymbolia patients and those who suffer from congenital or acquired analgesia: for, unlike the former, the latter never smile or laugh during pain testing procedures. And this can best be explained by the fact that in their case, unlike in the case of pain asymbolia, there is no feeling of pain that can serve as false alarm or mock threat that one recognizes by smile or laughter. So, phenomenological differences are, indeed, displayed in observational behavioral differences; but the main point is that these crucial behavioral differences are grounded on and are to be explained by the corresponding phenomenological differences or by the simple fact that only asymbolia patients feel pain or have the sensation of pain when they are harmfully stimulated.

As we have seen there is, indeed, plenty of clinical, experimental, and experiential evidence that clearly and quite convincingly proves that 
the sensation of pain or pain quality plays an important role in human total pain experience. To repeat, the central role being that of distinguishing pain sensations from non-physical pain, from other unpleasant sensations, as well as from other sensations that may be qualitatively similar to it, but are of different modality. If that is the case, then this evidence which strongly speaks in favor of the fundamental role of the sensation of pain or of the importance of pain quality, may well be used to loosen the force of one of the most powerful antisubjectivists arguments ever devised in favor of the functionalist interpretation of the true nature and structure of human pain experience. And the point is that this argument is the only one which allows functionalism to move from perpetual tedious defense of its position into quite unexpected vigorous offence. The main target of this argument is the very qualitative or phenomenal aspect of sensations which is so profoundly felt to be missing from the functionalist account; and the major point of the argument is to prove that - contrary to deeplyrooted subjectivist intuitions - this allegedly missing element, even when present and fully recognized, plays no essential role as far as the type-identity conditions for the sensory states are concerned. Ironically enough, pain - that paradigm case of the sensory state considered to be in principle resistant to functionalist analysis or decomposition - is taken to prove this point. For it is claimed, on introspective grounds, that various cases of pain are accompanied with qualitatively divergent or disparate sensations, and that - consequently - there is no common felt quality that can give unity to pain or constitute its type-identity. The conclusion drawn in favor of functionalism being that the unity or type-identity of pain is solely to be sought in the common or similar stimulus-response pattern displayed in all qualitatively different instantiations of this sensory state. This argument was for the first time put forward by Churchlands in the following way:

Consider the wide variety of qualia willfully lumped together in common practice under the heading of pain. Compare the qualitative character of a severe electric shock with that of a sharp blow to the kneecap; compare the character of hands dully aching from making too many snowballs with the pierc- 
ing sensation of a jet engine heard at very close range; compare the character of a frontal headache with the sensation of a scalding pot grasped firmly. It is evident that what unites sensations of such diverse characters is the similarity in their functional roles. The sudden onset of any of them prompts an involuntary withdrawal of some sort. Our reaction to all of them is immediate dislike, and the violence of the dislike increases with the intensity and duration of the sensation. All of them are indicators of physical trauma of some kind, actual or potential. All of them tend to produce shock, impatience, and vocal reactions of familiar kinds. Plainly, these collected causal features are what unite the class of painful sensations, not some uniform quale, invariant across cases. (Churchland, Paul, and Churchland, Patricia, 1981, pp. 125-126)

Similar argument devised to show that the essence of pain is not to be determined in qualitative or phenomenal terms, but rather in functionalist terms, is to be found in Michael Tye's book, The Metaphysics of Mind, where it is spelled in the following way:

Functionalists have been quick to point out that pain is not a state the essence of which can be defined in phenomenal terms. Pains vary enormously in how they feel. Consider, for example, the pain of a burn, a headache, a very loud noise to the ear, a pinprick, a bee sting. Nonetheless, such pains do at least typically elicit the same mental reaction, namely dislike. This leads to the thought that the characteristic of typically eliciting a reaction of dislike is part of the essence of pain. I say "part" here because there are other effects (and causes) that seem to many philosophers no less important to the classification of a given state as pain. Consider, for example, such typical causal relationships as these: resulting from bodily damage or trauma; giving rise to worry and distress; causing attempts to move the body away from the damaging stimulus; causing nursing of the relevant part of the body.

Functionalists have asserted that causal relationships like these themselves exhaust the essence of pain. According to function- 
alism, any sensory state type (and indeed any mental state type) can be defined via its causal connections with certain standard stimuli, certain other types of mental states, and certain standard behavioral responses. (1989, p. 91)

That pain, because of its qualitative variety, cannot be given unity through its common felt quality, is also argued by Norton Nelkin:

Though introspection cannot resolve many of the problems in this area, an appeal to the reader's introspection on this issue seems entirely legitimate: consider the significant differences among phenomena we do call pain phenomena (those experienced when cut, when suffering a toothache, or when having a headache). It is hard to understand what feeling they all share in common such that they are all pains. (1994, p. 329)

The argument advocated by Churchlands, Tye, and Nelkin to the effect that there is no common or uniform felt quality that would unite the class of pain sensations may, at first sight, appear as quite convincing or even conclusive, because it relies on the uncontestable introspective evidence that there is a striking qualitative difference between sensations that go under the heading of pain. But this argument will loosen its grip to the point of full release, the moment we realize that qualitative differences between pain sensations that Churchlands, Tye, and Nelkin refer to and ground their argument on, are actually intra-modal differences within the modality of pain: that is, the qualitatively distinct sensations of sharp pain and dull pain or sensations of stinging and burning pain are experiential determinates of the experiential determinable the sensation of pain or the modality of pain. As we have shown, patients who are unable to feel pain upon noxious stimulation may, nevertheless, be able to discriminate between sharp and dull or between stinging and burning qualitative character of the corresponding mechanical or thermal noxious stimuli. Here, the qualitative differences in the sensations felt are in fact intramodal differences within the modality of the sensation of touch or the sensation of heat. In other words, if sharp sensation or dull sensation is to be felt as pain, it has to carry the mark of pain quality or pain modality. It is this quality that unites pain sensations and sets them 
apart from other sensations that may be phenomenally similar to them, but are of different modality. Besides, the common felt quality that gives unity to pain sensations or makes of them a separate class of sensations is also something that sets them apart from other unpleasant sensations. Tye's claim "that the characteristic of typically eliciting a reaction of dislike is part of the essence of pain" is not only partial or deficient in functionalist terms, but also wanting in phenomenal terms, because it ignores the fact that there are other unpleasant sensations besides pain, and that pain need to be distinguished from them by its unique and distinctive felt quality. Similar remark has been made by Roger Trigg when he was considering possible answers to the question "Why do you dislike that sensation?"

To be told that it is because it is unpleasant is to have the dislike of the sensation merely reaffirmed. The answer 'Because it hurts' or 'Because it is painful' not only rules out many unpleasant types of sensation but explains why we dislike that sensation. Similarly the statement that a sensation 'is unpleasant, but isn't painful' is not a contradiction. It tells us that the sensation may be disliked, but nevertheless does not have that distinctive and insistent quality which marks off pain from other sensations. (1970, p. 26)

This again proves that, contrary to the anti-subjectivists claims made by Churchlands, Tye, and Nelkin, the role of pain quality or the concept of separate pain modality is to unite and distinguish the class of sensations that go under the heading or genus of pain. It also shows in what respect the common and distinctive felt quality of pain is the essential or indispensable component of our total pain experience and why that experience is not pain experience when that component is missing. As far as the functionalist arguments against subjectivism are concerned, the most that one can claim is that pain sensation comes to nothing when it is disconnected from the corresponding affective, cognitive, and motor machinery of our total pain experience. And this is something that is quite clearly and convincingly proved by the case of pain asymbolia patients. Namely, once we make the distinction between feeling pain and being in pain, and allow that one can feel pain without being in pain, it will become obvious and undisputable 
that all painfulness that is to be found in pain experience pertains to its affective, cognitive, and motor machinery. However, the affective, cognitive, and motor machinery that produces and carries whatever painfulness there is to pain must, nevertheless, wear on its sleeves the signature of pain sensation in order to count as genuine pain experience. So it seems that we must not only allow that pain without painfulness is possible, but must also make room for the opposite possibility: that of painfulness without pain. In other words, we might now be willing to claim that it should also be quite conceivable, and thus possible, for someone to go through and exhibit painfulness without feeling pain. But the point is that, like in the case of pain without painfulness, we need not at all engage ourselves in constructing bold imaginary scenarios or farfetched thought-experiments in order to see whether the case of painfulness without pain is possible. Namely, there is incontestable clinical evidence that such case exists, and this is certainly the shortest and most reliable way to prove that it is, indeed, possible.

The case that we are interested in was only recently disclosed and has been described as pain affect without pain sensation. This bizarre experience was found out during the neurological testing of the patient with selective lesion of the right primary (SI) and secondary (SII) somatosensory cortices: that is, exactly of those areas of the brain that are considered to be responsible for the processing of the sensory-discriminative components of pain or the precise spatial localization, temporal registration, intensity calibration, and qualitative characterization of the sensations of pain evoked by noxious or potentially noxious stimuli. "While sensory examination of the patient's right side was within normal limits, left-sided examination revealed hypoaesthesia of foot, leg and face and anastheasia of hand and arm ... In particular, thermal stimuli did not evoke any sensation" (Ploner, Freund, and Schnitzler, 1999, p. 212). When controlled, selective thermonociceptive stimuli were applied by means of cutaneous laser stimulation, the following results have been obtained:

... pain thresholds were $200 \mathrm{~mJ}$ for right hand and both feet. Evoked pain sensations were characterized as 'pinprick-like' and were well localized within $2-3 \mathrm{~cm}$. For left hand, up to an 
intensity of $600 \mathrm{~mJ}$, no pain sensation could be elicited. However, at intensities of $350 \mathrm{~mJ}$ and more, the patient spontaneously described a 'clearly unpleasant' intensity dependent feeling emerging from an ill-localized and extended area 'somewhere between fingertips and shoulder', that he wanted to avoid (italics mine). The fully cooperative and eloquent patient was completely unable to further describe quality, localization, and intensity of the perceived stimulus. Suggestions from a given word list containing 'warm', 'hot', 'cold', 'touch', 'burning', 'pinprick-like', 'slight pain', 'moderate pain', and 'intense pain' were denied nor did the patient report any kind of paraesthesia. ... Reaction times to laser stimuli on the right hand showed a bimodal distribution with medians at $400 \mathrm{~ms}$ and $1000 \mathrm{~ms}$. By contrast, stimulation of the left hand yielded exclusively long-latency responses with a median at $1426 \mathrm{~ms}$. (Ploner, Freund, and Schnitzler, 1999, p. 213)

As the authors of this study have pointed out, their results demonstrated, for the first time in humans, "a loss of pain sensation with preserved pain affect" (Ploner, Freund, and Schnitzler, 1999, p. 211). The more general implications of this phenomenon related to the assessment of the proper role and place of sensory-discriminative and affective-motivational components in human pain experience, they have summarized in the following way:

In the patient reported here, clinical examination and cutaneous laser stimulation revealed prolonged reaction times to painful laser stimuli, an elevated pain threshold, loss of sensory-discriminative pain component, and preserved motivational-affective dimension of pain. This clear perceptual dissociation was paralleled by an anatomical dissociation between affected lateral pain system and spared medial pain system. This pattern of impairment shows the essential role of SI and/or SII for the sensory-discriminative aspects of pain perception in humans. By contrast, detection of and reaction to painful stimuli as well as pain affect do obviously not require integrity of SI and SII. Nevertheless, damage to SI and SII produce hypoalgesia in our patient, suggesting interaction between medial and lateral pain 
system in normal pain experience. (Ploner, Freund, and Schnitzler, 1999, p. 213)

In this case of painfulness without pain we actually have a very clear and vivid picture of what pain experience comes to when it is deprived of its sensory-discriminative component, while its affective and behavioral components are kept intact. Namely, the subject who has lost sensory-discriminative capacities of pain is not any longer able to precisely localize the site of the noxious or potentially noxious stimuli applied to the part of his body. The most that he can do in this respect is to refer to an ill-localized and extended area 'somewhere between fingertips and shoulder'. But it means that one of the primary biological functions of pain has been lost: that of accurate localization of harmful or potentially harmful stimuli. "Such function appears reserved for pain as a signal of noxious challenge to the body surface which needs to be well localized for the purposes of removal of agent, projection of the injured site or inhibition by gentle rubbing or scratching" (Ochoa and Torebjörk, 1989, p. 593). The second major deficiency consists in the absence of the capacity of precise temporal registration of thermal damaging or potentially damaging stimuli because the subject displayed prolonged reaction times to painful laser stimuli. And that means that he is deprived of the capacity of instant detection of the noxious stimulus which may have disastrous consequences: namely, the stimulus can inflict damage to the part of the body long before the affective and motor machinery is put into action for removal of the agent or escape from it. The absence of precise calibration of the intensity of the noxious stimulus also speaks that one of the vital functions of pain has been lost. The subject was unable to describe the intensity of the perceived stimulus by any of the words suggested to him: 'slight pain', 'moderate pain', or 'intense pain'. But then one can say that he is deprived of the capacity to properly assess the extent and significance of the threat that the noxious or potentially noxious stimulus imposes to the integrity of his body. The fact that the subject could not specify in any way the quality of the perceived stimulus tells us that no information about the character or nature of the stimulus was available to him. Namely, he denied any further determination of the sensation felt as 'warm', 
'hot', 'cold', 'touch', 'burning', or 'pricking-like'. So he could not on qualitative grounds determine whether the sensation felt was of pain or not; nor he could on the ground of the quality of the sensation felt determine the nocuous or innocuous character of the stimulus. His dislike of the feeling that emerged upon cutaneous laser stimulation as well as his wish to avoid it were not motivated by the quality and intensity of the sensation felt: by that distinctive and insistent quality which marks off pain from other sensations. On the contrary, they were motivated by its pure or sheer unpleasantness. Paradoxically enough, in this bizarre case of pain affect without pain sensation, the only answer that one could get to the query - 'Why do you dislike that sensation?' - would be 'Because it is unpleasant'. It is true that to give such an answer is really to give no answer but merely to reaffirm the dislike of the sensation. It tells us nothing new or gives us no fresh information. But the point is that that is the only information available to the subject.

What the cases of pain without painfulness and painfulness without pain can teach us about the very concept of pain or, more to the point, about the very experience of pain? Should they be treated as cases of genuine pain or just as extreme aberrations that tell us nothing about the true nature of the phenomenon that we are interested in? Well, on the one hand, there is pure pain sensation, and on the other hand pure feeling of unpleasantness defying any further sensory specification. In the first case, pain comes to nothing in the sense that it doesn't carry any meaning for the subject and doesn't move (emotio) him in any way. In the second case, pain comes to such sensory indeterminacy that it looses all informational power with regard to the location, intensity, and nature or character of harmful stimuli. So, as far as two basic components of human pain experience are at stake, it is obvious that both of them are necessary, but that neither of them, taken by itself, is a sufficient condition for pain. For they give us real pain only when they work together. And this is how it should be, if Mother Nature has devised the pain system to serve its primary biological function: to give an organism basic sensory directions and informations concerning threatening or damaging stimuli and simultaneously 
move it in order to take action or resist action for self-protective purposes. 


\section{Not Caring for Pain}

Pain asymbolia is certainly the most striking and the most convincing reactive dissociation syndrome which proves that people can be completely indifferent to pain; that they may not care or mind for it at all; that they can feel pain and yet not show the slightest tendency toward any appropriate or typical motor and affective reactions to it. It is, of course, also the most vivid and dramatic evidence of what would happen to us if we were indifferent to pain; for it shows quite clearly that we would simply not be able anymore to protect in any way the integrity of our body and mind. As we have already suggested, pain asymbolia is the only reactive dissociation syndrome to be found in human pain experience that represents the clear case of thorough or total indifference to pain; moreover, it is the only clear-cut case in which pain is not felt anymore as unpleasant. Namely, if one peruses all paradigmatic cases of indifference to pain that are to be found in the relevant clinical and experimental reports, it will turn out that there are different ways in which people can be indifferent to pain. In other words, the attitude of indifference to pain epitomized in the responses, "I don't care", "I don't mind" or "It doesn't bother me", may well differ with regard to its scope and object; besides, it may, but need not mean that pain is not felt anymore as unpleasant; finally, it may well differ with regard to its major causes and more general neurological an psychological deficits on which it is grounded. In order to prove this point, I will at some length compare the indifference of pain displayed in pain asymbolia patients with indifference to pain that is to be found in lobotomized, cingulotomized and morphinized patients. But first all these cases of real indifference to pain, whatever their scope, objects and causes may be, should be set apart from those cases which are improperly treated as cases of indifference to pain.

One of the main reasons why, in the clinical literature, some cases of the lack or absence of typical pain behavior are, inadequately and misleadingly labeled or diagnosed as cases of the indifference to pain, is to be found in the fact they are actually cases of indifference to noxious or harmful stimuli, and not to pain. As the very word says, if 
one is to be indifferent to pain, one should be able to feel it, but not care or mind for it; not have a tendency to react to it in any way. On the other hand, one can be indifferent to noxious or harmful stimulation exactly because one doesn't feel pain upon such stimulation and, consequently, doesn't care or mind for it; doesn't show the usual behavior of withdrawal, attack, wincing, grimacing, complaining, or crying out. In the first case, indifference presupposes the presence of pain or pain-feeling, while in the second case indifference is due to the absence of pain in response to stimulation which would normally be painful. Transient indifference to noxious stimuli is displayed under various special conditions:

For example, severe noxious stimulation may go unnoticed during extreme excitement, anger, or fear, as in combat or sports. Absence of reaction may also be found in the apathy of extreme depression. States of dissociation, like hysteria and hypnosis, show unusual indifference to noxious stimuli as a main characteristic. Reports of self-mutilation involving severe bodily damage are not uncommon in psychotic episodes. (McMurray, 1955, p. 121)

Permanent and encompassing indifference to noxious or harmful stimuli is characteristic of the patients who suffer from congenital universal analgesia or as it is sometimes called congenital universal insensitivity to pain. As the latter term implies, these patients actually suffer from the loss of pain or pain-feeling throughout the body. But the loss or absence of pain upon the stimulation which would normally be painful - that is, upon the application of noxious or harmful mechanical, thermal, or chemical stimuli - need not, and in these patients doesn't mean, that they are unable to detect or recognize these stimuli. As I have earlier said, these patients are quite able to discriminate between sharp and dull stimuli or between stinging and burning stimuli. In other words, loss of pain or pain-feeling doesn't involve or imply complete somatosensory loss. The patients that we are interested in have sensations when they are noxiously stimulated, but the sensations evoked by this kind of stimulation are sensations of touch or of heat, and not of pain or of pain modality. In their case, there is the mismatch between the stimulus mode and the response 
mode that leads to the loss of specificity of a sensory modality. In other words, the original modality of sensations evoked by noxious stimuli is normally painful, but in cases of congenital analgesia these stimuli evoke non-painful sensations.

The fact that patients suffering from congenital analgesia or insensitivity to pain are, nevertheless, able to detect and discriminate noxious stimuli as sharp or dull, is exactly the source of deep confusion and serious misunderstanding that have led some authors to claim that these syndromes should better be treated as cases of congenital indifference to pain. Thus, Critchley who has reviewed the largest sample of reports on patients considered to suffer from congenital insensitivity to pain, has claimed that the alternative label, "congenital indifference to pain", is preferable because it avoids "the ambiguity of the term 'sensitivity' which might unwittingly suggest some loss of painfeeling" (1956, p. 741). But that the loss or absence of pain or sensation of pain is the distinctive mark of the patients that Critchley has put to scrutiny, stems from the very results of pain testing which he himself has presented. Namely, out of seven patients, no patient had headache upon histamine injection; two had a sense of throbbing and three had flushing with subjective warmth. Out of ten patients, no patient felt pain or discomfort upon prolonged muscle ischemia, and only one experienced a light feeling. Electric shock didn't evoke pain in four patients and they had not objected to faradic stimulation (Critchley, 1956, p. 740). However, for Critchley the decisive or crucial thing is that patients, who are in no way mentally retarded, hysterical, or psychotic, "present no objective anomaly of sensation. They can detect, identify and localize pinpricks, and can distinguish quite minor differences in thermal contacts" (1956, p. 737). The suggestion is that there is actually no loss of pain sensation but only loss of pain reactions to painful stimuli: "Experimental interventions which might be expected to give rise to pain provoke neither a verbal protest nor mimic responses, such as wincing, nor yet any vegetative reflex activity" (Critchley, 1956, p. 737). As only pain reactions are, according to Critchley, absent in the syndrome that he is considering, he is recommending that it should be treated as the syndrome of con- 
genital indifference to pain. And this is how he describes the distinctive marks of that syndrome:

The most remarkable feature in this syndrome is a typical lack of conformity between the feeling of pain as a discriminative quality of sensation, and the registration of distress, either overtly or automatically. Thus we have a dissociation between the various components of a normal pain-experience, i.e., (1) the qualifying notion of pressure, cutting, heat, etc., which constitutes the nature of pain; (2) the highly unpleasant sensation which constitutes the specific sense-datum of pain, and (3) the feeling tone of displeasure. The first component is cognitive in nature, while the second and third are affective. In cases of congenital indifference to pain the first component is intact, while the third is in abeyance, and perhaps also the second. This discrepancy is reminiscent of what may follow the operation of lobotomy, and also the pain asymboly of Schilder and Stengel; it differs from these conditions, however, in that they also entail a defect of the second component, i.e., the specific pain-feeling. This component may or may not be intact in cases of congenital indifference to pain. (Critchley, 1956, p. 742)

For Critchley the fact that patients or subjects can readily, upon being noxiously stimulated, feel a pinprick and distinguish between sharp and dull, is taken as a reliable and conclusive sign that they feel pain: that the sensation of pain has been evoked by the noxious stimulus. As patients are care-free and display no pain reactions, their indifference is interpreted as indifference to pain. Of course, if we are to speak at all about the indifference to something, the object of indifference has to be recognized and distinguished from other objects. But, as I have already pointed out, the stimulus - a pinprick - can be detected and felt as sharp with full intensity of its sharpness, and yet not be felt as pain or as the sensation which falls under the modality of pain. As this is true for patients which suffer from congenital analgesia or congenital insensitivity to pain, these clinical labels should be kept in use for the description and diagnosis of the relevant syndrome, and not the inappropriate and misleading label of congenital indifference to pain. 
As has been shown, the confusion between indifference to noxious stimulation and indifference to pain basically stems from the fact that no clear distinction is made between two quite different sensory capacities: that one is able to feel the noxious stimulus - the pinprick and that one is able to feel the sensation of pain. This confusion or the obliteration of this fundamental distinction has also led some authors to improperly treat cases that are actually the cases of indifference to noxious stimulation as cases of pain asymbolia or as cases of real indifference to pain. We have already mentioned, in another context, the case of the patient with right parietal and insular lesion whose ailment was improperly treated as pain asymbolia syndrome. If you remember, the patient studied by Masson et al. (1991, pp. 668-670), did not mind and did not display any reactions to noxious stimuli applied to his right hand, and was improperly treated as the patient suffering from pain asymbolia simply because he was able to feel pinprick and distinguish between sharp and dull. If this patient displayed indifference, which he did, the indifference was the indifference to noxious stimulation and not indifference to pain. Same misunderstanding and the same improper use of the words is to be found in the case of two patients studied by Rubins and Friedman and treated by them as paradigm cases of pain asymbolia or indifference to the very sensation of pain:

The predominant feature shown by our patients was the pain asymbolia, as described by Schilder. ... Two patients stated repeatedly that the pinprick didn't hurt even after prolonged application and to the point of drawing blood. ... Other stimuli such as heat or cold, produced a similar result. One patient would hold a lighted match until her fingers would almost burn without dropping it. (1948, p. 565)

These are, indeed, frightening examples of complete indifference or total absence of reactions to extremely noxious stimuli. But the question is whether these two patients have felt pain when they have being exposed to such stimuli. Now, this is how Rubins and Friedman have evaluated the sensation of pain and sensibility in other modalities: 
Except for the asymbolia, the other modalities of superficial and deep sensation showed little disturbance. Touch and thermic stimulation were well perceived and to the same extent throughout the body. Passive movement of joints and position of digits in space were normally recognized.

Pain sensation as evaluated by our routine criteria - namely, ability to distinguish between sharp and dull on application of a pointed object or to perceive sharpness with the same intensity - was normal. Subject to the same fluctuation, pinprick was felt as equally sharp wherever applied and distinguished from a blunt stimulus. (1948, p. 566)

As Roger Trigg has observed, the problem with the evaluation of pain sensation that Rubins and Friedman have used, is that they "do not see the distinction between being able to feel a pinprick and being able to feel sensation with "pain quality" (1970, p. 73). Elaborating further this basic distinction, Trigg has come to the following conclusion:

If a patient is not numb they (Rubins and Friedman) assume that he can feel pain, and all interest must then center on the patient's ability, or lack of it, to react normally to pain. ... It is in no way self-contradictory to report sensations of sharpness normally, and yet to deny pain. Just because such sensations may normally possess the quality of pain, it does not follow that they must always. Rubins and Friedman can be accused of paying too much attention to the nature of the stimulus, and not enough to the nature of sensation, as described by the patient. Because a stimulus, such as severe pin-prick, is normally painful, they assume that all that is required for the occurrence of pain is that the patient perceive the stimulus. They forget that it is possible for the sensations produced by the same stimulus to vary. (1970, pp. 73-74)

The confusion between indifference to noxious stimulation and indifference to pain can also be detected in the study conducted by Weinstein, Kahn, and Slote on a group of patients "who appeared inattentive to disabled parts and sides of the body, were mute, hypo- 
kinetic, and who showed the phenomenon of pain asymbolia. It was considered that these forms of behavior were in some degree forms of implicit denial of illness" (1955, p. 235). That pain asymbolia and, consequently, indifference to pain, are wrongly attributed to these patients, becomes clear from the way in which Weinstein, Kahn, and Slote have described the general condition of pain asymbolia allegedly present in 15 , out of 20 patients, that they had put to scrutiny:

When stimulated vigorously with the point of the pin or in some other noxious fashion, they did not react to the stimulus as though it was painful or unpleasant. Usually, they did not pull away, attempt to brush aside the pin, cry out, wince, or grimace. Similarly they did not heed threatening gestures. Even on the occasion when a patient may act as if a stimulus was painful, on being questioned, he would deny that it hurt. ... It was not simply a matter of impaired sensation, because many times the patient could distinguish sharp and dull and hot and cold and would complain of pain in another context. (1955, 240)

The description of one particular case diagnosed for pain asymbolia is given within the general pattern described above:

Vibratory, proprioceptive, and tactile sensibility was normal. The patient was able to distinguish heat from cold and sharp from dull in routine fashion but showed marked pain asymbolia. When stimulated forcibly with a point of a pin or with very hot objects, he gave no indication of feeling pain. Pressure over the Achilles and elbow tendons gave the same result.

The pain asymbolia persisted throughout his hospital stay of a month and for six weeks following discharge. Even though pricked on the point of drawing blood, the patient gave no evidence of perceiving pain. On one occasion a very hot stimulus produced a slight burn. When asked about this on the following day, the patient denied that the examiner had burned him, even though a mark was still present. He confabulated that another doctor who looked like the examiner had accidentally dropped 
a cigarette ash on him. On another occasion he cut himself shaving but denied both the cut and any pain.

The patient presented an overly polite, servile manner. He invariably answered with "Sir", and even called his wife "Ma'am". He always smiled when anyone else smiled. (1955, p. 243)

But why attribute pain asymbolia to this patient, when in the very case report it is explicitly stated that the patient gave no evidence of perceiving pain; no indication of feeling pain. The distinctive mark of pain asymbolia patients is exactly the fact that they are able to perceive or feel pain, but that it doesn't bother them; that it is nothing to them; and that they don't have event the slightest tendency to react to it. When they are aware of their plight, they do not confabulate, but try to rationalize their behavior or are astonished by it. They tend to underrate the intensity of their pain and they smile or laugh, but only during pain testing, and not when anyone else smiles. They smile at the very pain that they feel or perceive, because they feel or perceive it as a mock threat or false alarm. We have to remember that the lobotomy patient observed by Paul Brand also smiled at her pain, because it was not anymore the source of great suffering and didn't meant anymore the threat for her: "She smiled sweetly and chuckled to herself. 'In fact, it's still agonizing. But I don't mind"' (1997, p. 210).

The case of the patient studied by Weinstein, Kahn, and Slote should, as far as his loss of pain reactions is concerned, actually be treated as the case of indifference to noxious or harmful stimulation, and not to pain. By its very meaning, indifference to noxious stimulation presupposes the sensory registration of such stimulation; otherwise, it would not be indifference, but sheer undetectability or pure loss of sensitivity with regard to such stimuli. So, the fact that this patient is able to distinguish between sharp and dull and between hot and cold fits well to this description. On the other hand, as the very word "asymbolia" suggests, the patients who really suffer from pain asymbolia are not able to attach proper meaning or significance to the pain that they in the literal sense feel or perceive. If they were not feeling 
or perceiving pain, upon being noxiously stimulated, there would be nothing to which they were unable to attach proper meaning or significance, or smile or laugh at. The very application of the term "pain asymbolia" would not makes sense, and in the case of the patient studied by Weinstein, Kahn, and Slote it doesn't make sense. The fact that there are conspicuous behavioral differences between this patient and the patients who really suffer from pain asymbolia is important, because it shows that there are observational differences between indifference to noxious stimulation and indifference to pain. In other words, it cannot be objected that the difference between these two indifferences makes no difference and should, thus, be discounted or eliminated. Although in both cases pain reactions are absent, the absence or presence of pain or pain sensation is, indeed, the difference that makes differences in behavior. It is a difference that makes one smile or laugh; that makes one to be, self-reflexively, astonished by one's own smiling or laughter; and, finally, it is a difference that makes one to seek explanation of one's own bizarre behavior in terms of rationalization, and not in terms of confabulation.

Drawing the clear distinction between indifference to pain and indifference to noxious stimulation is also important for precise description, diagnosis, and explanation of the loss of pain reactions in some psychiatry disorders: particularly in some cases of severe depression and in psychotic persons prone to catatonic schizophrenia and selfmutilation. In this context the study of Hall and Stride is of special interest, because they have carefully examined the sensation threshold, the pain perception threshold, and the pain tolerance threshold in 256 neurotic and depressive patients of both sexes and various ages. The results of the measurements of these three different thresholds have shown that, in general, "patients classified as depressed tend to have a high pain tolerance. ... Over $80 \%$ of those patients who did not report pain, and of those who did not react to pain, fall into this category" (Hall and Stride, 1954, p. 52). But Hall and Stride have noticed that there are two different ways in which depressed patients arrive at a high pain tolerance measure:

In one patient, described clinically as a recurrent endogenous depression, even the perception of warmth occurred beyond the 
mean point of pain perception for the total group. Verbal report of pain did not occur at all, the intensity of the stimulus at maximum being described as merely warm. The depression here seems to have, at least temporarily, reduced the verbalperceptual and, perhaps consequently, the motor response system to an inert state in which stimuli are not adequately discriminated.

Another kind of high pain threshold is sometimes found in several of the patients classified as involutional-type depressions. ... One patient in this category did not report pain even at the maximum intensity, but, on being asked to describe the nature of the sensation, he said: 'Well, it was like a lighted cigarette end being held against my forehead'. This type of patients will frequently describe a sensation as 'burning' or 'very hot', without, however, making any admission that it was at all what they meant by pain. There is probably very little, if any, difference in perceptual discrimination in these patients from that of other patients or normals. They do, however, differ markedly in their attitude toward the stimulus, in their evaluation as painful or not painful. This is demonstrated also by the fact that these patients report the stimulus as perceptibly warm as early in the scale as most patients of similar age. (Hall and Stride, 1954, p. 52)

In the case of the patient who is suffering from endogenous depression the evidence, as far as the feeling or perception of pain is at stake, is quite clear or unequivocal. The patient doesn't feel any pain even at the maximum intensity of the thermal stimulus; and although the patient is able to detect the heat-stimulus at its maximal intensity, due to extremely lowered sensitivity, the nature of the stimulus is inadequately discriminated as merely warm. The patient suffering from involutional or agitative depression is, on the contrary, quite capable to adequately perceive or discriminate the heat stimulus of maximal intensity as burning or very hot; besides, his sensitivity is quite normal because he reports the stimulus as perceptibly warm as early in the scale as any other patients or normals. The only question is whether he feels pain and is not reacting to it emotionally, or is he 
not feeling pain at all? The patient's explicit reports would suggest that he doesn't feel or perceives pain at the maximal intensity of heat stimulus, although he is able to recognize the stimulus as burning or very hot; moreover, the patient is denying that by "burning" or "very hot" he means pain. However, Hall and Stride insist on the fact that there is little, if any, difference "in perceptual discrimination in these patients from that of other patients or normals" (1954, p. 52). This carries the implication that the patient is feeling the same kind of sensation as normal people and that only the emotional reaction is absent. In other words, the difference from normal people would only consist in the fact that the patients "differ markedly in their attitude towards the stimulus, in their evaluation of it as painful or not painful" (1954, p. 52). But, as Roger Trigg has remarked, the major question is not related to the stimulus, but to the sensations caused by the stimulus, and the phrase "evaluation of it as painful" is, in this context, highly peculiar and ambiguous:

As Hall and Stride are concerned to emphasize that these patients have the same feelings as others ... 'painful' is presumably not intended to refer to quality of a sensation, but is used as a synonym for 'distressing' or some such word. The claim is, therefore, that these patients are feeling the same kind of sensation as normal people but do not have any emotional reaction. ... The authors' use of 'painful' to mean 'distressful' may be usual when what is described as 'painful' is a situation, but in connection with sensations 'painful' is used to refer to a pain-quality - even if it may carry the idea that we are distressed by it. What they are doing is to define pain as a 'sensation that is disliked', and this has the result that they ignore their patient's inability to feel the distinctive quality of pain. (1970, p. 69)

If Hall and Stride have spoken of the indifference to noxious or harmful stimulation all ambiguities, misunderstandings and, what is most important, wrong descriptions of the facts could have been avoided. At one point they are inclined to speak of "indifference to pain stimulation", but this term may also be misleading because it suggest that the stimulation is of the kind that evokes pain sensation that one 
is indifferent to. So again, the term "indifference to noxious stimulation" is there to dissipate all possible misapprehensions and misnomers.

Keeping in mind the difference between two indifferences - the one to noxious stimulation and the other to pain - may also help as to better comprehend the absence of pain reactions in catatonic schizophrenia that almost regularly involves the tendency toward self-mutilation or auto-aggressiveness:

These patients often hurt or mutilate themselves without showing the slightest apparent sign of pain. Bender and Schilder divided these reactions into two types, according to the dominant mental symptoms of patients they studied. Those with stupor but without marked tension showed no reaction whatsoever to pinching, pinprick, or blows but rapidly and vigorously withdrew on faradic stimulation. Patients with pronounced "tensions" frequently reacted to prolonged application of painful stimuli - i.e., pinching or electric shock - but in an inadequate and local way. For instance, there might be squirming or athetoid movements of the fingers, sometimes spreading up to the arm, occasionally accompanied with change in respiratory rate with tears in the eyes or with stiffening of the body. The response is rather a postural attitude than an action of escape or defense. One of our patients, a man with catatonia, would role his head from side to side whenever he was painfully stimulated but never withdrew his limbs. This perverted reaction disappeared when his catatonic state cleared up. (Rubins and Friedman, 1948, p. 555)

Rubins and Friedman are quite apodictic in their claim that patients described above do not feel pain and at best react to noxious stimulation in an inadequate and local way. The only exception to the rule the brisk and vigorous withdrawal on faradic stimulation - may be attributed to the sheer muscular contraction. And yet Rubins and Friedman are inclined to compare schizophrenic patients to the patients suffering from pain asymbolia as initially described by Schilder and Stengel. In order to see whether this comparison is grounded, we 
will in more detail present the case of one such patient studied by Trelles. Namely, pain asymbolia was attributed to this patient on account of his catatonic states and particularly on account of his frequent and regular acts of self-mutilation which were not followed by any sign of pain or suffering. For instance, the patient would frequently burn his hands with cigarettes and would deny any pain; he would punch himself vigorously without feeling any pain and would say that he had done that in order to check out " that he is not afraid of pain and that he is brave"" (Trelles, 1978, p. 6). Neurological examinations of this patient have shown that he is able to distinguish different types of stimulations used for the exploration of superficial sensitivity: tactile, thermal, and painful. However, he would never show suffering, pain, or reactions of defense. When thermal stimulation is applied - very hot water - or sometimes a profound prick with syringe, he says that it hurts, but neither withdraws the hand nor displays any reactions of escape:

The patient is not disturbed that stimulations are incessantly repeated, each time more strongly (pinpricks), and has no intention of protecting himself; the patient obviously knows that these stimulations produce pain and suffering and says: "I know doctor that this hurts, that this pricks and produces pains ... how could it not hurt? ... someone would suffer ... but I don't feel ...”. (Trelles, 1978, p. 7)

Trelles is asking himself whether the case of this patient should be treated as the case of true or real pain asymbolia? The patient is not feeling pain nor displaying pain reactions during self-mutilation, so that it seem that on such occasions he is indifferent to noxious stimulation rather than to pain. However, Trelles is pretty convinced that in the experimental setting the patient feels or perceives pain upon being noxiously stimulated, because he regularly and reliably perceives the pinprick, pinch or very hot or very cold stimuli. But is this sufficient for the appearance of the sensation of pain? The patient knows that these stimuli hurt, and is aware that someone else would suffer. However, of himself he says: "but I don't feel ...". Unfortunately, we are not told what the patient doesn't feel. My guess is: pain. 
Now that we have drawn and amply illustrated the difference between the indifference to noxious stimulation and indifference to pain, let us consider in more detail the differences that exist between various indifferences to pain. As I have said, these differences may relate to the scope of indifference; to its proper object, and to the fact whether pain is or is not anymore experienced as unpleasant. In order to illustrate these differences, we will at some length consider the indifference to pain displayed in lobotomy patients, and will argue that, unlike the indifference to be found in pain asymbolia patient, the one found in lobotomized patients is of limited scope; it is not directed at the very sensation of pain, but rather at its significance, and need not mean that pain is not anymore experienced as unpleasant.

The purpose of the operation of prefrontal lobotomy (or leucotomy) is to cut the nerve fibers which connect the front of the brain with the rest. "Prefrontal lobotomy, either unilateral or bilateral, is performed by transecting the cerebrum in a plane identified by the coronal suture and the sphenoidal ridge and just anterior to the lateral ventricle. It is estimated that Brodman areas 8, 9, 10, 11, 32, 46 and 47 are isolated by these procedures" (Hardy, Wolff, and Goodel, 1952, p. 307). The operation has been quite widely used in thirties, forties, and fifties to relieve chronic pain of functional or organic origin, and intractable pain in fatal diseases (quite often when a patient has become addicted to "pain-killing drugs"). As the cutting of the nerve fibers connecting frontal regions of the brain with the rest has turned out to have farreaching effects on personality, surgeons became reluctant to perform it anymore.

Let us now consider some of the case histories of the lobotomy patients as they are presented in Freeman's and Watts' famous book Psychosurgery in the Treatment of Mental Disorders and Intractable Pain:

Case 9

A woman of hysterical temperament began at the age of 16 to complain of abdominal pain so persistently that she accumulated a series of 12 to 18 abdominal operations. ... Following a trivial head injury, she complained so bitterly of pain in the 
head that a subtemporal decomression was performed. From 1934 to 1936 she was confined to bed because of agonazing pain in the back and limbs. ... On account of exaggeration of complaints with very little anatomic substrate, a diagnosis of conversion hysteria with poly-surgical addiction was made.

Prefrontal lobotomy was performed November 30, 1936, by the Moniz technique. The change brought about in this patient's fear reaction was immediate and remarkable. On the second postoperative day the patient was alert and fairly talkative and admitted that the sensitiveness that she had formerly felt over the spine has disappeared. ... The patient turned over on her back, gingerly at first, then with greater confidence. When her lower limbs were manipulated and forced into extension for the first time in many months, there was much crepitation. The patient cried out with pain, but nevertheless seemed to enjoy having her legs straightened out once more.

When the patient returned to the neurology clinic for follow up visits, she appeared to be in good spirits and reported that she felt fine. However, when we asked her specifically about her back, she said: "This back of mine hurts so I can hardly walk." We could find no muscle spasm or rigidity in the spine, although twisting about in various directions brought tears to her eyes and she complained bitterly of pain. After the examination, she dressed herself and walked without showing any evidence of pain.

Comment: When Mrs. S. first told us she had pain six months after operation we feared a relapse. Then it gradually dawned upon us, as we observed this patient's behavior, that she still had pain but was no longer disabled with it; she was not afraid of it, and she could work and earn a living in spite of it. (Freeman and Watts, 1950, pp. 354-357)

Case 301

Age 55. Hypochondriasis. Pain in back since girlhood, worse since birth of first child 28 years ago, and unbearable for last three years. ... Prefrontal lobotomy of the standard type, per- 
formed on March 1, 1945, was followed by alleviation of back pain. ... By April, 1947, the patient ... had resumed many of her household activities. She says the pain is still there, but it does not bother her. (Freeman and Watts, 1950, p. 358)

Case 165

Age 62. Hypochondriasis. Disabling pain since 1931, especially in back and hip. The patient suffered a series of financial reverses after the stock market crashed, and in 1931 developed severe pain in the back and left hip with radiation down the leg. There was also pain across the chest in the region of his heart. ... A standard prefrontal lobotomy was performed April 14, 1943, after which he stopped complaining about his back and sedation was discontinued. He lives a quite existence at home and mentions his pains only when asked about them. (Freeman and Watts, 1950, pp. 358-359)

Case 280

Tabes dorsalis. Ten year's disability with girdle pain and lightning pain in legs. ... A standard prefrontal lobotomy was performed on December 4, 1944, after which narcotics were discontinued. He continued to have attacks but described them as twinges which he was able to control with aspirin. ... The neurologic signs of tabes are as obvious as ever. His perception of pain is as keen as before, but his reaction to pain lacks the emotional component that was disabling. (Freeman and Watts, pp. 366-367)

As far as the cases of intractable pain of functional origin are concerned, Freeman and Watts have come to the following conclusion: "The fear of pain in the patients described in this chapter has been the outstanding and disabling feature. After prefrontal lobotomy, these patients can apparently feel pain just as acutely, but they are no longer afraid of pain, nor concerned over the possible consequences. Their perception of pain is intact but their reaction to pain is brief and reduced in intensity. The emotional component is attenuated and the fear of pain is no longer disabling" (1950, p. 360). With respect to the cases of pain of organic origin, they observed that "preoccupation 
with pain dominated the life of the individual to the extent that he could no longer carry on his ordinary activities. At the same time, however, he could often be distracted by interesting events in his vicinity and would not mention his distress. ... There is less obvious relationship in these cases between pain and the emotional status, but the affective charge is highly important. When it is reduced by lobotomy, the pain may become tolerable, opiates can be discontinued ... and the individual may return to work" (Freeman and Watts, 1950, p. 360 ).

Their view on the general effects of lobotomy, Freeman and Watts describe in this way: "The attitude was different. Fear seemed to have gone. The pain was still present, but it was a sensation rather than a threat" (1950, p. 353). In other words, "lobotomy does not interfere with the perception of pain, nor does it abolish the normal reaction to pain. It does reduce the persistent, obsessive, emotional substrate of continued pain. We would compare this situation with the dynamite charge which is detonated by a percussion cup. The emotional substrate represents the explosive, and when this is removed, the percussion cup can go off any number of times without resulting in a great explosion. Case 280, the tabetic, spoke of his twinges" (Freeman and Watts, 1950, p. 372).

However, the case-histories presented by Freeman and Watts do not reveal the very important data concerning the intensity of pain before and after the operation of prefrontal lobotomy, nor do they disclose any facts related to the pain sensation threshold and reaction threshold of the patients, either preoperatively or postoperatively. For this kind of information, we have to turn to the study conducted by Hardy, Wolff, and Goodel on a series of 38 prefrontal lobotomies, performed by Dr. Bronson Ray in the New York Hospital, on patients with intractable pain. As these authors report, "in 21 out of 38 persons, following lobotomy, some relief of pain appeared to have been achieved in some way; of these, 17 admitted experiencing pain only when asked; four had no pain at all" (1952, p. 310).

Measurement of pain threshold, by the use of the thermal radiation method, has been carried on eight patients before the operation. "The 
average of all these measurements was $206 \mathrm{mc} . / \mathrm{sec} . / \mathrm{cm} .2$... This is a mean value within the normal range. ... Pain thresholds measurements were made in ten patients post-operatively. In two patients the pain threshold was observed to be lowered immediately following operation. In one of these who was observed again two weeks post-operatively the pain threshold had returned to the pre-operative level. In three patients who had been measured pre-operatively the post-operative pain threshold was unaltered. In five patients who were not measured pre-operatively, measurements one week or more postoperatively gave pain thresholds within the normal range, 200 to 230 $\mathrm{mc} . / \mathrm{sec} . / \mathrm{cm} .2$. The mean of all post-operative pain thresholds measurements was $209 \mathrm{mc} . / \mathrm{sec} . / \mathrm{cm} .2$, a value which is not significantly different from the pre-operative level". These results have led Hardy, Wolff, and Goodel to come to the general conclusion that, "although the pain threshold may show temporary alterations, the average of the pain threshold before and after operation is essentially the same" (1952, p. 322).

The measurements of pain intensity in five patients before and after the operation have shown that the preoperative pain was, in general, "of low intensity with brief episodes of more intense pain accompanying a disturbance of a diseased part. However, at no time was any patient completely free of pain. These two factors characterized the pain picture in these patients" $(1952$, p. 313). As far as the postoperative data related to the pain intensity are concerned, it has turned out that in only one patient the intractable pain was completely eliminated, while in other four "the pain was reduced on the average and in maximum value. However, they were at no time pain free" (1952, p. 313). The examination of the postoperative reaction thresholds of 23 lobotomized patients - that is, the wincing and pulling of their heads in response to thermal radiation - has also shown that there is no major alteration in reaction to new or fresh or short-lived noxious stimulation, or that the reaction can sometimes be more vigorous than before the operation (Chapman et al., 1950, pp. 386-392). This is in accord with bedside observations that, "when suddenly moved or turned over, the patient, who until this time may have been relaxed and tranquilly resting in bed, may cry out vigorously with pain from 
bombardment of additional noxious impulses from his damaged bone or joint structure" (Hardy, Wolff, and Goodel, 1952, p.320).

When results of the measurements of pain intensity, pain sensation, and pain reaction thresholds in lobotomized patients are taken into account, it seems that the major effects of the operation are not to be sought in the changes in pain perception, intensity discriminations or reactions to momentary harmful stimuli. According to Hardy, Wolff, and Goodel, the most important changes are manifested in affect and attitude towards pain: "The lobotomized subjects became indifferent to low intensity pain which, though perceived, evoked few protective reactions. The attitude was epitomized in the response, 'Yes, I feel the pain, but it doesn't bother me'" (Hardy, Wolff, and Goodel, 1952, p. 316). These subjects also "exhibited a freedom from anxiety about pain, did not anticipate its occurrence and lacked an interest in recall and description of pain experienced in the past. Each painful experience was dealt in its own terms as a fresh experience. They showed little concern about the implications of pain as regards damage to the body or threat to life. ... Indeed, these subjects exhibited in many ways, notably concerning the topic of pain, a flattened affect if not actual apathy. When noxious stimulation of high intensity was suddenly imposed upon them experimentally or by some surgical procedure, despite immediate vigorous reactions, these subjects promptly ceased to exhibit evidence of pain, 'forgot' their recent experiences, and turned to the casual reading of a newspaper or idly looking about" (Hardy, Wolff, and Goodel, 1952, pp. 316-317).

It is also characteristic for lobotomized patients that "lack of complaint and failure to call attention to their plight and needs were striking. They failed not only to complain of their spontaneous pain but also of their needs, such as personal nursing care, need of urine bottle, bedpan, or the adjustment of uncomfortable dressing. When incontinent of feces they were indifferent to odor. It spread about their persons and beds" (Hardy, Wolff, and Goodel, 1952, pp. 316-317).

Although the operation of prefrontal lobotomy for intractable pain has no uniform effects, there are still some standard or definite patterns of reactions characteristic for all lobotomized patients. And the first 
thing that emerges is the fact that such patients, though not minding the pain of their disease, feel and dislike other pains which may occur for a moment. The measurement of their pain sensation and pain reaction thresholds supports this observation which is very important because it shows that lobotomized patients are using the concept of pain properly: that is, under standard stimulation conditions followed by typical pain behavior. In other words, it cannot be claimed that these subjects are confused about or that they have even forgotten the very meaning of the word "pain".

The second characteristic thing is related to the radical change in their readiness to complain of pain. Before the operation, the pain has absorbed their whole attention - they were obsessed by it - but after the operation they tend to speak about their pain and complain of it only when they are explicitly questioned. This seems to be related to the fact that their concern has narrowed down to the immediate present; that they see and care little for anything that is outside the actual present.

The third behavioral pattern characteristic of post-lobotomy patients can be described, in general terms, as a "mitigated "readiness to respond' to external and internal stimuli" (Barber, 1959, p. 439). This decreased responsiveness coming from personality alterations is manifested in its extreme form in total apathy, and in its lesser forms in a decrease in worry and concern or in impaired ability to elaborate a persisting attitude or mood.

The three behavioral patterns presented above seem to show that in lobotomized patients the sensation of pain remains, but that the dread, anxiety, or fear which accompanied their permanent pain have gone, so that they bother little or not at all about it, although any sudden change in their pain, or any new pain, is liable to evoke an immediate (but only transient) response. Is this to be taken to mean that for postlobotomy patients their permanent, but disregarded pain, is not anymore the object of dislike? In other words, has it ceased to be unpleasant for them anymore?

If an answer to these questions is to be given, we have to draw the distinction between two kinds of emotional reactions to pain which 
are not always clearly distinguished. On the one hand, there are emotional reactions which take the very sensation of pain as its object, and depend upon its quality, intensity, location, and duration. On the other hand, there are emotional reactions which are directed at the significance of pain, and which primarily depend upon the meaning that we attach to the pain that we feel. Dislike, unpleasantness, or distress belong to the first category of emotional reactions to pain, while anxiety, dread, or fear are to be reckoned among those that belong to the second category of emotional reactions to pain. Although these two kinds of emotional reactions to pain usually go together, it does not follow that the changes in the emotional reaction of the second kind will inevitably bring about changes in the emotional reactions of the first kind. In other words, the anxiety, fear, or dread related to the meaning of the pain that we presently feel may go, while the unpleasantness or distress that we feel over it might well remain the same. For instance, I am suffering from glaucoma which was, fortunately, diagnosed in its early stage and is under the medical control. Still, if I were to feel a sharp, intense pain in my eyes I would become frightened, because I know that this kind of pain may be the sign of the deteriorating state of my eyes. My fear would alarm me and I would certainly go to visit my ophthalmologist. If he was to reassure me that there is nothing wrong with my intra-ocular pressure, and that there are no significant changes in my visual field, my fear would be gone. But the pain could still be unpleasant or distressing, although I would not be anymore concerned or worried about it, and would find the way to live with it.

The major effect of the operation of prefrontal lobotomy has been exactly the disappearance of the anxiety, fear, or dread that the subjects have felt over their sustained, intractable pain. As Freeman and Watts have put it: "The attitude was different. The pain was still present, but it was a sensation rather than a threat" (1950, p. 353). And this change in the attitude of lobotomized patients towards their pain seems to be the outcome of the more general change in their emotional setup, reflected in their "flattened affect" and in the fact that their concern has narrowed down to the immediate present. Because they do not attach to their permanently present pain the meaning or 
significance that they used to ascribe to it before the operation, it does not bother them anymore or they do not care or mind for it anymore. But it does not follow that they do not dislike it anymore or that it has stopped to be unpleasant for them. As we have shown, one may not be bothered by something anymore or may not care or mind for it anymore, once it has been robbed of the meaning or significance that it used to carry for a person, although the unpleasantness or distress may remain the same. Of course, this will depend very much on the intensity of pain; but, as we have seen, in the case of lobotomized patients the pain was mostly - though sustained - of moderate intensity. And where it was severe, one might explain their not being bothered by it or not minding it by the fact that they have reached the state of total apathy.

Generally speaking, the consideration of the clinical, psychological, and behavioral profile of the lobotomized patients does not lend support to the view that for them pain is not anymore an unpleasant state of affairs or that it has stopped to be the object of their dislike. If that is true, it means that we are not compelled to give up our well-entrenched intuition that pain is inherently unpleasant on the account of the reports of lobotomized patients who claim that they still feel pain, but that it does not bother them anymore or that they don't mind or care for it anymore. In other words, indifference to pain need not mean that the ongoing pain is not anymore disliked or not anymore felt as unpleasant, but may only mean - as the case of lobotomy patients shows - that one doesn't care or mind for it because it is not anymore the object of anxiety, fear, depression, or frustration related to its past and long-term future implications. Let me remind you once again that the patient observed by Paul Brand speaks of her persistent pain condition as still agonizing, but also as something that she doesn't mind:

As a German neurosurgeon who had performed many prefrontal lobotomies once told me, "The procedure takes all the suffering out of pain." Stages one and two of pain, the signal and message stages, proceed without interruption. But a radical change in stage three, the mind's response, transforms the nature of the overall experience. (1997, p. 211) 
Indifference to their persistent pain condition is, in lobotomy patients, the consequence of their inability to give any lasting or long-term significance to the ongoing chronic pain: “... subjects with prefrontal lobotomies or leucotomies have severe disruption of their abilities to cognitively assess the meaning and implications of chronic pain. However, these subjects have normal or even lowered pain thresholds and retain their ability to experience both sensory and early affective components of pain" (Coghill et al, 1999, p. 1941). Wade et al. speak of the first stage of pain affect (or pain unpleasantness), and the second stage of pain affect having to do with emotions related to longterm implications of pain, and they claim that nearly all of the affective verbal descriptors of the McGill Pain Questionnaire refer much more to the immediate threat and unpleasantness of pain:

For example, the words 'tiring', 'exhausting', 'sickening' and 'suffocating' refer to unpleasant intrusive aspect of pain closely associated with sensory qualities and words such as 'fearful', 'frightful', or 'terrifying' refer to the immediate threat associated with pain. In contrast, answers to questions about how depressed, anxious, and frustrated one feels in relation to one's pain rely more on reflection concerning the past and long-term future implications of a persistent pain condition. (Wade et al., 1996, pp. 163-164)

That lobotomized patients are, indeed, able to experience both sensory and early affective components of pain has been already quite convincingly shown. So, additional piece of evidence will be presented just to give as a better insight into to the real scope, proper object, and genuine character of the indifference to pain displayed by these patients:

It should be emphasized that the leucotomized patient is able to respond normally to nociceptive stimulation. Hardy et al. (1952, p. 316) have reported that "some patients, although ostensibly tranquil before being asked about their pain, overreacted with a show of grimacing and fears when their attention was focused upon it by a direct question concerning its quality and its intensity (emphasis added). ... Apparently, when the 
leucotomized patient is directly asked to report on his pain, he "focuses his attention" on and "thinks about" the ever-present nociceptive stimulus in his body and, when thus reacting to it, often shows discomfort and suffering and almost always reports a "sensation of pain". However, when the patient is not directly asked to report on noxious tissue condition, he does not "attend" to it or "think" about it to the same extent as before the operation and, when not thus reacting to it, does not appear to be "in pain" ... (Barber, 1959, p. 439)

From this additional as well as all other evidence that we have, it is obvious that indifference to pain displayed by lobotomy patients is strictly limited to the ongoing or persistent pain; that its object is not the very sensation of pain, but its lasting meaning or significance; and that this kind of indifference doesn't imply that pain is not anymore disliked or experienced as unpleasant. Indifference to pain of this limited scope and character is fundamentally the consequence of the more general deficit or incapacity of lobotomy patients to pay longer attention and give lasting meaning to any novel, threatening, discomforting, or disgusting stimuli. Indifference to pain confined to ongoing persistent pain is also characteristic for patients who have undergone cingulotomy for the relief of chronic intractable pain. After this kind of psychosurgery, cingulotomized patients, like lobotomized patients, admit that they still have pain, but that it is not distressing, bothersome and doesn't worry them anymore. But these patients are similar to lobotomized patients in another respect because they also react vigorously to any new inflicted pain:

Numerous clinical studies report decreases in the suffering, distress, and analgesic requirements that follow frontal leucotomies and cingulotomies for the treatment of the intractable chronic pain. Many of these same studies, however, also document a persistence after neurosurgical lesions of exaggerated withdrawal responses, wincing, and grimacing, which are evoked by even trivial clinical procedures such as pinprick and venipuncture. (Talbot et al., 1995, p. 124) 
Paradoxically enough, the relief of pain and suffering is achieved in the face of exaggerated behavioral responses to noxious stimulation. But this only proves that indifference to pain is, both in lobotomized and cingulotomized patients, of limited scope. Unlike lobotomy, cingulotomy doesn't have serious consequences for the personality of the patient and doesn't impair seriously his cognitive capacities. However, a recent study of 18 patients has shown that it impairs, to significant extent, some domains of attention and executive control:

Most patients reported mild improvements in pain severity, although none reported a complete absence of pain. Most patients reported overall benefit from cingulotomy and being less bothered by pain. ...

Cingulotomy patients showed weaker performance than control subjects on measures that reflect the ability to focus on and sustain attention to the task at hand ... had greater impairments on attention/executive measures of response intention, generation, and persistence. (Cohen et al., 1999, p. 450)

So it seems that the major effect of cingulotomy was to attenuate the tendency of patients to continuously respond, emotionally and behaviorally, to the ever present pain, and this squares well with their reports that the pain is still there but is not bothersome or is so to the lesser degree. In this respect, cingulotomy patients have, like lobotomy patients, deficits in spontaneous concern and rumination about their ongoing persistent pain, but for both groups of patients the following is true:

... (they) can experience the immediate threat of pain once it is brought to their attention. In contrast, asymbolia patients appear incapable of perceiving the threat of nociceptive stimuli under any circumstances. (Price, 2000, p. 1771)

In other words, solely in the case of pain asymbolia patients we come across the total or complete indifference to pain, because only these patients don't care or mind for any pain inflicted on any part of their bodies, and only these patients do not experience it anymore as unpleasant or something that is disliked per se. And one must say that even in the indifference to pain displayed by morphinized subjects, 
the unpleasantness of pain may still be present. Namely, one recent study of the effects of oral morphine on cold pressor tolerance time came to the following conclusion:

Subjects who received oral morphine ... showed increased pain tolerance time in comparison with the control subjects receiving the active placebo (diphenhydramine). The morphine dose of $0.429 \mathrm{mg} / \mathrm{kg}$ produced a significantly longer tolerance than did the active placebo. Although a significant increase in tolerance time in the cold pressor task was observed, there were no significant reductions in either pain intensity or unpleasantness ratings during cold pressor for either the morphine or diphenhydramine groups.

Morphine and diphenhydramine produced similar changes in mood. ... These mood changes included ... reductions of anxiety and fearfulness. (Cleeland et al., 1996, p. 260)

In pain asymbolia patients thorough indifference to pain is due to the fact that these patients are entirely incapable to experience the immediate threat and unpleasantness of pain, despite their capacity to detect sensory features of pain. Consequently, they are not able to give to their pain any long-term or lasting meaning or significance. But if pain without any painfulness is possible, as the case of asymbolia patients unequivocally shows, should we abandon our deep conviction that pain is inherently unpleasant? Should we revise our concept of pain, and not treat pain as inextricably bounded to unpleasantness? I think that we should not, for the case of pain asymbolia is rather to be taken as the case which clearly shows us what pain comes to when it is deprived of unpleasantness: that it comes to nothing and serves no biological purpose. To put this point into other words and make the final conclusion: the indifference to pain displayed in pain asymbolia patients is so thorough and complete that it reaches the point where one starts to smile and laugh at pain that one feels or perceives. 


\section{C and All That Fibers}

When we were considering the basic neural mechanisms and structures that subserve pain as the major human self-protective system, we have mentioned the important role that $\mathrm{C}$ and $\mathrm{A}$ delta nociceptive fibers play in it. Their physiological specialization has been precisely determined; the distinctive qualitative character of the pain that their activity typically elicits vividly described; and, finally, the motor reactions that their firing immediately evokes were quite clearly and distinctively specified. Now, the interesting and quite surprising thing is that $\mathrm{C}$ fibers are, of all sensory fibers detected in sensory physiology, certainly the most popular sensory units among philosophers, while unfortunate A delta nociceptive fibers are almost completely ignored in philosophy of mind. Namely, everybody who is familiar with central issues related to contemporary materialism must have heard for $\mathrm{C}$ fibers, because the firing of these neural structures or sensory fibers was exactly picked out as the neural (physical) activity with which the experience of pain is to be identified: pain $=\mathrm{C}$ fiber firing. The reason why that should be done was, as far as I know, never clearly stated ever since these fibers were introduced in philosophy as - either referential or functional - identificational targets of pain. Data about their morphological, physiological, and psychological distinctive properties were conspicuously missing, and reports of the relevant electrophysiological and psychophysical studies were almost never mentioned. As Daniel Dennett, one of the few philosophers who had an insight into neural mechanisms of pain, has remarked: during the heated debate between materialists, functionalists, and subjectivists in sixties and seventies of the last century, the term "C fiber" or "C fiber firing" seem to have lost, for philosophers, "its empirical anchoring in neuroanatomy and became a philosopher's wild-card referring expression for whatever physical event 'turns out to be identical with' pain" (1978, p. 450, fn. 4).

Some philosophers have located these peripheral afferent fibers in the brain; some have denominated them as "pain fibers", obliterating the distinction between the physiological specialization and psychological specificity of sensory fibers; finally, they have even been debarred of 
any sensory-discriminative role in pain experience. But worse was yet to come in the guise of the final philosophical verdict on poor $\mathrm{C}$ fibers. Van Gulick was bold enough to speak about the discovery of Cfiber firing as "the standard philosophical candidate for the neural basis of pain, despite its total empirical implausibility". And the same attitude is to be found in David Lewis: "So if the state pain is C-firing, to take a toy example, then the distinctive quale of pains would be the property; being an event of C-firing" (1995, p. 141). One should only remind oneself of the analgesia from which leprosy patients suffer due to the destruction of peripheral nociceptors $-\mathrm{C}$ fibers included - to see how these statements or proclamations are unfounded.

So, from being uncritically introduced into philosophy, C fibers have ended up by being uncritically rejected. But this is just a case, as will be shown, of philosophical disregard for the relevant facts and has no bearing for the really important theoretical issues related to the connection of the firing of $\mathrm{C}$ fibers and the experience of pain. The really important claim and a real challenge is to be found in Joseph Levine's statement that:

... there seems to be nothing about C-fiber firing which makes it naturally 'fit' the phenomenal properties of pain, any more than it would fit some other set of phenomenal properties ... the identification of the qualitative side of pain with $\mathrm{C}$-fiber firing (or some property of C-fiber firing) leaves the connection between it and what we identify it with completely mysterious. One might say, it makes the way pain feels into a merely brute fact. $(1983$, p. 357$)$

In order to see whether psychophysical connections are really doomed to remain just a matter of brute, unintelligible correlations, I will first examine at some length the basic physiological properties of $\mathrm{C}$ fibers as well as the distinctive psychological consequences of their activity. As far as these properties of $\mathrm{C}$ fibers are concerned, two important things have been conclusively established through the relevant electrophysiological and psychophysical studies: first, that these fibers along with A delta fibers - selectively or preferentially respond to 
noxious or potentially noxious stimuli (stimuli which, if prolonged, would damage the tissue); second, that their activity causes dull or burning pain, while the firing of A delta fibers elicits sharp or pricking pain. So let us consider how these properties of $\mathrm{C}$ and A delta fibers were determined, and whether they can help us to see that there is not only a brute correlation, but also an intelligible connection between the firing of these fibers and pain experience.

$\mathrm{C}$ fibers, like A delta fibers, are to be found in all mammals and are widely distributed in skin as well as in deep tissue; they not only outnumber A delta fibers, but also all other sensory fibers, and this is particularly the case for human beings. From the morphological point of view, C fibers as well as A delta fibers belong to the group of small-diameter primary afferent neurons: the sensory nerve units which consist, at least, "of a receptive terminal located in peripheral tissue, a peripheral afferent fiber that represents a conductive link, a cell body in a dorsal root or trigeminal ganglion, and central terminals in the spinal cord or medulla" (Perl, 1984, p. 25). However, C fibers differ from A delta fibers in two important respect: first, they are not covered by cuffs of myelin, a laminated fat-protein insulating material; second, they transmit nerve impulses more slowly then A delta fibers. Namely, their conduction velocity ranges from 0.5 to $1.5 \mathrm{me}-$ ters per second, while that of A delta fibers ranges from 6 to 30 meters per second. This means that it can take more than a second for the nerve impulses conducted by $\mathrm{C}$ fibers to reach the spinal cord from the stimulated foot, while the nerve impulses transmitted by A delta fibers from the same part of the body have reached it "long before".

The physiological specialization of $\mathrm{C}$ fibers and A delta fibers, like the specialization of all other sensory fibers, has been studied in two ways: first, by dissecting out the nerve and recording its activity under certain range of natural stimuli with gross (silver) hook electrodes; second, by inserting percutaneously tungsten needle microelectrodes into the nerve in order to record impulses from single fiber evoked upon delivery of natural stimuli to the skin or deep tissue. The former method was used in animal experiments, while the latter has enabled physiologists to carry electrophysiological experiments in awake human subjects. This invasive electrophysiological technique, known as 
microneurography, was developed in late 1960s (Vallbo and Hagbarth, 1968) and has revolutionized the field of sensory physiology, because it has provided the experimental tool by which it was possible to record impulses from single sensory units in an intact human subject. In other words, it has largely materialized the desire "... to compare both stimulus and sensation with the messages which pass up the sensory nerve fibers" (Adrian, 1931).

The electrophysiological techniques described above have made it possible to determine, in precise and reliable manner via the natural stimulus-electrical response strategy, the adequate stimulus for sensory fibers: that is, the stimulus to which they best or particularly respond. It has been found out that, within the range of $\mathrm{A}$ delta and $\mathrm{C}$ fibers, there are fibers which preferentially react to strong mechanical stimuli (like stroking, scratching, or pinpricking), to temperatures above $45^{\circ} \mathrm{C}$ or that below $23^{\circ} \mathrm{C}$, and to irritant chemicals (histamine, for instance). Although these stimuli are of quite different modes they have, as Sherrington has long ago noticed, "in relation to the organism one feature common to all its components, namely, a nocuous character" $(1948$, p. 227). That is to say, they are "either frankly tissue damaging or are of such intensity that any small increase or long maintenance of stimulation results in tissue damage" (Price and Dubner, 1977, p. 307). This is the reason why in sensory physiology stimuli of this kind were labeled noxious or potentially noxious stimuli, and why the fibers which have been discovered to respond preferentially to such stimuli were classified as nociceptive fibers - the existence of such fibers being anticipated by Sherrington several decades ago (1948, pp. 229-230). For instance, fibers of this type will react to temperatures around $48^{\circ} \mathrm{C}$ at which nerve substance begins to suffer injury; or, they will respond to the pressure of the thorn or needle just below the pressure sufficient for the thorn or instrument to break into the skin. Moreover, their firing will activate withdrawal or flexion reflexes, and thus initiate one of the basic protective actions of the organism with respect to the stimuli that are of such intensity as threatens damage to the skin (Melzack and Wall, 1988, pp. 102-103).

Among A delta fibers, there are fibers which best respond to only one kind of noxious or potentially noxious stimuli. Thus, there are A delta 
fibers which preferentially react to mechanical noxious stimuli, and are consequently labeled A delta mechano nociceptive fibers; there are also fibers which were found to respond mostly to the noxious heat stimuli, and are called A delta heat nociceptive fibers; finally, fibers which were discovered to react best to chemical irritants are classified as A delta chemo nociceptive fibers. It is characteristic for $\mathrm{C}$ fibers that they respond to all three noxious or potentially noxious stimuli - mechanical, thermal, and chemical - and this is the reason why they are designated as $\mathrm{C}$ poly-modal nociceptive fibers (Melzack and Wall, 1988, pp. 86-87). Quite recently, novel classes of C fibers have been discovered - the so-called "silent" C nociceptive chemo fibers - which do not respond to immediate noxious stimuli, not even to severe one, but are particularly responsive to the slaw changes in the state of inflamed peripheral tissue, or start to react to chemicals released after the injury has been inflicted to joints, muscles, or nerves (McMahon and Koltzenburg, 1990). Their activity is usually followed by tenderness (primary and secondary hyperalgesia and hyperaesthesia) which spreads around the damaged part of the body, making it extremely sensitive even to completely innocuous stimuli, and greatly diminishing movement and manipulation that would disrupt the process of repair and recovery after injury.

In order to avoid misunderstanding or conceptual confusion, it should be remarked that the classification of a certain group of sensory fibers according to their physiological specialization or the stimulus to which they best respond, is not meant to imply that they do not react at all to other stimuli or that other groups of fibers cannot react to stimuli for which they show greatest responsiveness. The notion of the 'adequate stimulus' for receptors and fibers of the sensory system - the notion which is considered to be a biological principle or law is only to be understood as the ability of the sensory unit effectively and reliably to distinguish between different stimuli in the impulses it sends to the central nervous system. This conception of the adequate stimulus is clearly stated in Sherrington's original definition of the physiological specialization of sensory receptors and fibers:

The sensorial end-organ is an apparatus by which an afferent nerve fiber is rendered distinctively amenable to some particu- 
lar physical agent, and at the same time less amenable to, i.e. shielded from, other excitants. It lowers the value of the limen of one particular kind of stimulus, it heightens the value of the limen of stimuli of other kinds. (1900, p. 995)

The merits of this definition of receptor and fiber specialization in terms of the lowest limen (or threshold) for the particular stimulus are twofold: first, it doesn't exclude the possibility that stimuli other the one to which the receptor or fiber is best adapted can elicit its activity; second, it allows that other receptors or fibers can react to that stimulus. Thus, in the case that we are interested in, the physiological specialization of the $\mathrm{C}$ and A delta fibers as nociceptive sensory units is determined according to the following criterion: "the ability of the sensory unit effectively and reliably to distinguish between noxious and innocuous events in the signals it provides to the central nervous system" (Burgess and Perl, 1973, p. 59). There are, indeed, A delta mechanical nociceptive fibers which respond only when the stimulus intensity is noxious or nearly noxious (Burgess and Perl, 1973, pp. 62-63); but there are also A delta and $\mathrm{C}$ nociceptive fibers which are activated by thermal and mechanical innocuous stimuli. So, what really makes them nociceptive sensory units is the fact that they respond with higher frequency to noxious as opposed to innocuous stimulation of the skin or other tissue or that they show systematic differences in the discharge patterns for these two stimuli. On the other hand, there are law-threshold mechanoreceptors that are preferentially excited by the non-damaging mechanical disturbances of the skin, but which can also respond to mechanical noxious or potentially noxious stimuli. However, careful studies along the lines of natural stimulus-electrical response strategy have shown that they do not display any systematic differences in the discharge patterns for these two stimuli; and this is to be taken as a proof of the inability of individual mechanoreceptors "to provide appropriate information for distinguishing noxious from innocuous mechanical stimuli" (Burgess and Perl, 1973, pp. 60-61).

In the early 1980 s, the method of human microneurography was supplemented by the technique of intraneural microstimulation, and this has greatly enhanced psychophysical studies whose aim was to relate 
the activity of single afferent fibers or bundles of such fibers to the sensory judgments concerning the quality, temporal profile, magnitude, and localization of the sensations evoked by that activity. Namely, it has been found out that the same microelectrode inserted percutaneously into the peripheral nerves of awake subjects in order to record the afferent discharges of sensory fibers delivered by natural stimuli, can be used to stimulate electrically mechanoreceptive or nociceptive units so as to elicit 'elementary sensations' with distinct qualities, temporal characteristics, and felt as though originating in a discrete, mono-focal area of the skin (Torebjörk and Ochoa, 1980; Ochoa and Torebjörk, 1983; Wall and McMahon, 1985; Torebjörk, Vallbo and Ochoa, 1987; Ochoa and Torebjörk, 1989). The technique of intraneural microstimulation has made it possible to use a stimulus which selectively activates nociceptive units, by bypassing cutaneous (peripheral) receptors whose activity induced by natural or electrical stimulation "unavoidably co-activate unknown numbers of units of imperfectly established physiological identity" (Ochoa and Torebjörk, 1989, p. 594). In order to appreciate the technical achievements and research potentials of this method, it should be stressed that only in late 1960 s the distinguished sensory physiologist has made the following statement: "To stimulate a single fiber in an intact human subject, to prove satisfactorily that only that fiber and no other has been stimulated, and to record a meaningful sensory judgment is an almost incredibly difficult technical feat, and it will be a long time before unequivocal evidence can be obtained" (Sinclair, 1967, p. 12).

Before the psychophysical studies based on the technique of the intraneural microstimulation can be carried out, the physiological type of the sensory units which are to be examined with regard to the psychological consequences of their electrical stimulation has to be precisely identified. The first step in this direction is to insert the microelectrode "manually through the skin into an underlying nerve trunk $\ldots$ and to deliver trains of weak electrical stimuli while gently adjusting its position", till it has, "reached an intrafascicular site where INMS evoked a weak, mono-focally projected sensation" (Ochoa and Torebjörk, 1989, p. 585). The area or the site where the sensation evoked by INMS was felt by the subject is denoted as the projected 
sensory field. When this is established, the electrode is switched to the recording mode so as to determine the receptive field of the sensory unit which has been previously stimulated: that is, the field from which a sensory unit can be activated by natural stimuli. This is done by delivering such stimuli to the cutaneous field where the sensation was projected in order to record intraneurally by microneurography the responses of sensory units. When the unitary receptive field is localized, its area is outlined with ink on the skin and the physiological type of the sensory unit whose activity is recorded is identified on the basis of its stimulus-response characteristics: that is, according to the stimuli to which it preferentially responds. Needle electrode is optionally inserted in the receptive field to stimulate the sensory fiber, and its conduction velocity is then "calculated from measurement of latency and conduction distance between stimulating and recording sites" (Ochoa and Torebjörk, 1989, p. 585). Finally, proof that the fiber stimulated and the fiber recorded are one and the same,

... can be obtained by 'marking' the stimulated fiber: prolonged, high frequency intraneural microstimulation can render that single ... sensory fiber hyperexcitable. ... Upon intraneural microrecording this fiber will either discharge spontaneously or will generate a burst in response to a triggering pulse. Impulses evoked from the receptive field of that particular unit only will now interfere with or trigger activity in the hyper-excitable unit. ... Thus, it can be certified that a recorded unit with defined receptor characteristics and conduction velocity is identical with the unit stimulated in the nerve. (Torebjörk and Ochoa, 1980, p. 445)

The psychophysical studies that we are particularly interested in were carried out on "seventy-one C poly-modal nociceptors supplying glabrous and hairy skin in limbs of awake human volunteers", and their physiological type was identified, "on the basis of cutaneous stimulus-response characteristics recorded intraneurally by microneurography" (Ochoa and Torebjörk, 1989, p. 583). For this purpose, "natural mechanical stimuli (stroking, scratching, pinpricking), heat (contact of a glowing match) and occasionally histamine (intra-dermal injection ...) were given to the cutaneous field, where sensations were 
projected. Having localized the unitary receptive field (RF) and classified the receptor according to established criteria, the receptive field was mapped ..., and its area was outlined with ink on the skin" (Ochoa and Torebjörk, 1989, p. 585). C nociceptors in glabrous skin of the hand were polymodal "in the sense that they all responded to noxious mechanical and heat stimuli. A few units tested also responded to histamine injection and became spontaneously active thereafter. The receptive fields were small, usually $2 \times 2$ to $3 \times 3 \mathrm{~mm}$. Conduction velocities ranged from 0.5 to $1.5 \mathrm{~m} \mathrm{~s}-1$ " (Ochoa and Torebjörk, 1989, p. 591). All the units to be tested psychophysically were also "marked" by the standard methods described above. During psychophysical studies,

... the subjects had no clues as to exactly when intraneural stimuli were given, or what stimulus parameters were used. ...They were asked to describe in their own words the qualities and temporal profiles of sensations evoked by INMS, and to map directly on a real size picture of the hand the sites and sizes of the skin areas where sensations were projected. ... If the subjects had difficulties in naming the sensations, they were presented with a multiple choice questionnaire, composed from typical verbalizations collected in previous studies. (Ochoa and Torebjörk, 1989, p. 586)

Microneurography and INMS were carried out on five healthy subjects, ranging in age from 33 to 51 years. Fourteen experiments were performed in the median nerve at elbow level. ... Eight experiments were performed in the ulnar nerve, and one experiment in the superficial radial nerve at wrist level. A few experiments used the posterior cutaneous nerve of the forearm ..., or the peroneal nerve at knee level ..., or just above the ankle. ... A total of seventy-one $\mathrm{C}$ poly-modal nociceptor units with receptive fields in glabrous (thirty-seven units) and hairy (thirty-four units) skin of the hand, forearm, leg or foot were sampled in this study. (Ochoa and Torebjörk, 1989, p. 584)

Results of these studies have shown that pain evoked as threshold sensation during weak INMS delivered in cutaneous nerve fascicles 
"was regularly projected superficially to the skin. ... The quality of pain projected to the glabrous skin was often described as dull (thirteen experiments) or less frequently as burning (five experiments). By contrast, pain projected to hairy skin of the dorsum of the hand, forearm or lateral calf was typically reported as burning (all six experiments)" (Ochoa and Torebjörk, 1989, p. 589). As reported separately, "the subjective experience of focal pricking or stinging pain was found to correlate with excitation of A nociceptor units" (Ochoa and Torebjörk, 1989, p. 587), and such pain is projected to a punctuate area of the skin, whereas the burning or dull pain evoked by the stimulation of $\mathrm{C}$ nociceptors is projected to a significantly larger areas of the skin.

C locognosia tests devised to determine the degree of accuracy to which subjects can locate a painful event in the glabrous skin of the hand based on the input from $\mathrm{C}$ nociceptive fibers alone, have shown "the remarkable matching between the sensory projections (projected field) of dull or burning pain evoked by INMS, and the innervation territories (the receptive field) of $\mathrm{C}$ nociceptor fibers alone. ... It was found that the mean error in localizing a hot stimulus during A fiber block was $7.5 \mathrm{~mm}$ in the fingers ... and $10.5 \mathrm{~mm}$ in the palm of the hand" (Ochoa and Torebjörk, 1989, p. 593). Thus, it appears

... that the $\mathrm{C}$ fiber system can provide useful input for fairly accurate localization of noxious events, at least in the glabrous skin of the hand. ... It seems obvious that accurate cerebral localization function requires very refined circuitry. ... Such function appears reserved for pain as a signal of noxious challenge to the body surface which needs to be well localized for the purposes of removal of agent, projection of the injured site or inhibition by gentle rubbing or scratching. (Ochoa and Torebjörk, 1989, p. 597)

As far as the temporal profile of the painful sensations evoked by the intraneural microstimulation of $\mathrm{C}$ nociceptive fibers is concerned, experiments have shown that both dull and burning pain were sustained sensations, without intermittency: 
In other words, subjects could not detect the frequency of intraneural stimulation. At very low $(1 \mathrm{~Hz})$ frequency of stimulation usually no sensation was felt during a $5 \mathrm{~s}$ train. At $3 \mathrm{~Hz}$ gradual build-up of pain was noticed, often following a long latency of the order of 2-3 s relative to the onset of the stimulus train. With higher frequencies $(5-30 \mathrm{~Hz})$ the build-up of pain was faster, and the magnitude of pain increased proportionally to the stimulus frequency. (Ochoa and Torebjörk, 1989, p. 589)

The last point shows that the modulation of impulse frequency in a single sensory unit may be enough to signal intensity of a stimulus. Indeed, "firing frequency is translated into intensity of continuous percepts (pressure, pain), but determines the frequency of intermittent percepts (flutter-vibration)" (Torebjörk and Ochoa, 1980, p. 447). However, it should be stressed that the sensory modality does not change with frequency. Namely, the psychophysical studies based on the intraneural microstimulation of sensory fibers of different morphological and physiological type, have proved the quantal nature of elementary sensations evoked by the activity of these fibers:

When a sensation first became detectable during intraneural stimulation, it typically remained invariant within a certain current range with regard to quality, temporal profile, subjective magnitude, location, and the size and shape of the projection area. A gradual increase of stimulus intensity above threshold for the first sensation usually lead to discontinuous recruitment of additional sensations with new and discrete projections, rather than to continuous growth of intensity and projection area of the first sensation. New sensation were therefore abruptly recruited at individual stimulation thresholds, according to the all-or-none principle, and each could be characterized by its specific quality, typical temporal profile and spatially distinct projection area. ... We interpret these quantal changes in the evoked sensory experience as the subjective counterpart to electrophysiological recruitment of separate single sensory units. (Torebjörk, Vallbo, and Ochoa, 1987, p. 1522) 
Generally speaking, it seems to me that the review of the results of the psychophysiological and electrophysiological studies related to the examination of sensory consequences of the intraneural microstimulation of $\mathrm{C}$ nociceptive fibers, fully supports the conclusion reached by the authors of these studies that there was "remarkable matching of physiological unit type (C poly-modal nociceptor) with subjective quality of evoked sensation (dull or burning pain). Further, there was remarkable spatial matching of receptive field of given $\mathrm{C}$ nociceptor with projected field of the pain sensation evoked from the $\mathrm{C}$ recording site by INMS delivered at threshold intensity for conscious sensation" (Ochoa and Torebjörk, 1989, p. 583).

The psychophysical evidence purported to show that the sensation of pain is closely connected to the firing of nociceptive fibers (both of $\mathrm{A}$ delta and $\mathrm{C}$ type) is, indeed, impressive; but it need not, and most probably will not, in an important respect impress philosophers who are mainly concerned with the hard problems of consciousness related to the connection between the firing of $\mathrm{C}$ and $\mathrm{A}$ delta nociceptive fibers and pain. Of course, they will be eager to admit that strong correlations between the activity of certain neural structures and the experience of pain have been established: and they will certainly recognize that these correlations are not only of general sort related to experiential determinables, but also highly specific with regard to experiential determinates, because they associate particular kinds of pain - that of burning or dull quality, and that of pricking or stinging quality - to the activity of particular types of nociceptive fibers (the former to $\mathrm{C}$, and the latter to A delta fibers).

However, they will be quick to remark that exactly at this stage of our knowledge of the specific psychophysical connections we have reached the point where the explanatory gap problems or the hard problems of consciousness most pressingly impose themselves upon the theoretically more demanding mind. For they will claim that so far no property of the relevant sensory fibers has been discovered that would show us that the tight connection between the firing of these fibers and pain experience is intelligible, and not just a matter of brute, unexplainable (un-transparent) correlation. In other words, they will be convinced that no property has been disclosed that would in 
any way help us to give satisfactory answers to the hard problems of consciousness related to the firing of $\mathrm{A}$ delta and $\mathrm{C}$ nociceptive fibers: why it evokes pain, rather than some other sensation? why it evokes pain, rather than no sensation at all? why the firing of A delta nociceptive fibers elicits pricking or stinging pain, rather than dull or burning pain? why the firing of $\mathrm{C}$ nociceptive fibers induces dull or burning, rather than pricking or stinging pain? And satisfactory explanation, according to Levine, is precisely to be considered as a matter of removing alternatives:

I want to know why some event occurred, or why some object manifests a particular property; why this and not that. ... That is, we must be able to see why if the explanans is true then the alternative events could not have occurred. Anything less leaves us explanatorily frustrated. (1991, p. 38)

So, if these explanatory demands are taken into account, it might well appear that what has at most been explained by the electrophysiological and psychophysical studies presented above, is just the causal role of pain: its functional property of being typically or standardly induced by noxious or potentially noxious stimuli. For we now know, on the basis of strong and incontestable electrophysiological and psychophysical evidence, that this role is performed by $\mathrm{A}$ delta and $\mathrm{C}$ nociceptive fibers because these sensory units, unlike other sensory units, preferentially respond exactly to those stimuli which typically or standardly cause pain. However, it will be immediately pointed out, that there is more to our concept of pain than its causal role:

... there is its qualitative character, how it feels; and what is left unexplained by the discovery of C-fiber firing is why pain should feel the way it does! For there seems to be nothing about C-fiber firing which makes it naturally "fit" the phenomenal properties of pain, any more than it would fit some other set of phenomenal properties. Unlike its functional role, the identification of the qualitative side of pain with $\mathrm{C}$-fiber firing (or some property of C-fiber firing) leaves the connection between it and what we identify it with completely mysterious. 
One might say, it makes the way pain feels into merely a brute fact. (Levine, 1983, p. 357)

But to the theoretically naive and philosophically untroubled mind, it may well seem that - contrary to Levine - we have already discovered something about $\mathrm{C}$ fiber firing which makes it naturally "fit" the sensation of pain. For we have learned, on independent electrophysiological grounds (via the technique of intraneural micro-recording of the activity of sensory fibers during the delivery of natural stimuli), that this firing is distinctively evoked by noxious or potentially noxious stimuli. And are not these stimuli considered by us as adequate and appropriate for pain? Is it not true that any definition of pain will have to refer to them? Consider only the one given by the IASP Subcommittee on Classification, and the commentaries that follow it:

An unpleasant sensory and emotional experience associated with actual or potential tissue damage, or described in terms of such damage.

Each individual learns the application of the word through the experience related to injury in early life. Biologists recognize that those stimuli which cause pain are liable to damage tissue. Accordingly, pain is that experience which we associate with actual or potential tissue damage. (IASP Subcommittee on Classification, 1986, p. 217)

So, when we are informed through the meticulous psychophysical studies which rely on the powerful and exquisitely precise technique of intraneural micro-stimulation, that there is a tight connection between the firing of $\mathrm{C}$ nociceptive fibers and pain, it will seem to us that we can understand or explain that connection. Namely, the physiological specialization of these fibers which consists in their preferential or distinctive responsiveness to noxious or potentially noxious stimuli makes their connection to pain intelligible, because pain is exactly conceived by us as being distinctively evoked by such stimuli. Thus, pain and the firing of $\mathrm{C}$ nociceptive fibers share one distinctive property in common, and we can on that ground see why 
they are tightly connected or why the firing of these fibers naturally "fits" the sensation of pain, and not some other sensation.

Generally speaking, it seems that we are fully allowed to rely on something that is already or as such intelligible to us in order to bestow intelligibility to something that doesn't wear that mark or distinction on its sleeves. The intelligibility of the relationship between $\mathrm{C}$ nociceptive fiber firing (as well as A delta nociceptive fiber firing) and pain will be a kind of conferred or second-order intelligibility, established via the first-order or conferring intelligibility of the functional-phenomenal relationship between noxious or potentially noxious stimuli and pain. If the relationship between pain and noxious or potentially noxious stimuli is as such intelligible to us, the role of these stimuli as adequate or appropriate stimuli both for pain and for the activity of $\mathrm{C}$ and $\mathrm{A}$ delta nociceptive fibers will install, through conceptual mediation, homogeneity between phenomenal and physiological concepts which are standardly thought to be inherently heterogeneous. Thus, conceptual heterogeneity or categorial difference between experiential and physical (neurophysiological) predicates will not feature anymore as the main legitimate source for further explanatory demands with regard to the established psychophysical connections. In other words, the way will be opened for the theoretical integration of the experiential into the physical.

This doesn't mean at all that the a priori analysis of phenomenal states in functional terms is a prerequisite for a satisfactory or adequate psychophysical explanation of qualia. The connection between noxious or potentially noxious stimuli and pain is, in the sense to be explained later, intelligible as such, and it will bestow intelligibility to the tight connection between the firing of $\mathrm{C}$ and $\mathrm{A}$ delta nociceptive fibers established by psychophysical studies, if it is found out through the relevant electrophysiological experiments that this firing is distinctively evoked exactly by those stimuli which are considered by us to be intelligibly attached to the experience of pain. Thus, the discovery that $\mathrm{C}$ and $\mathrm{A}$ delta fibers distinctively or preferentially respond to noxious or potentially noxious stimuli will not only reveal to us neural structures that perform the causal role of pain, but will also lead us to see that there is an intelligible connection between the firing of 
these fibers and pain experience. In other words, functionalist considerations of phenomenal states will play the role of hermeneutical mediators in psychophysical explanations, and not - like in Lewis' standard model (1966, pp. 17-25) - the role of aprioristic tools that are supposed to open the only way to adequate or satisfactory psychophysical explanations.

But it will be said that we have just postponed the hard problems of consciousness related to the connection between the firing of $\mathrm{C}$ and $\mathrm{A}$ delta nociceptive fibers and pain; that we have merely relegated them to the functional-phenomenal level where they will be directed at the connection between noxious or potentially noxious stimuli and pain. For the further questions will now be: why these stimuli give rise to pain, and not to some other sensation? why they give rise to pain, rather than to no sensation at all? As Levine has remarked, "it still seems that we can ask why the kind of state that performs the function performed by pain, whatever its physical basis, should feel the way pain does" (Levine, 1983, p. 358). And similar theoretical challenge is to be found in Chalmers: "We know that conscious experience does arise when these functions are performed, but the very fact that it arises is the central mystery. There is an explanatory gap ... between the functions and experience, and we need an explanatory bridge to cross it" (Chalmers, 1995, p. 203).

If no satisfactory reply can be given to the demands for further intelligibility between certain functions and the experience of pain, this will certainly cast doubt on the conferred or second-order intelligibility of the connection between the firing of nociceptive fibers and pain, and we will be back to square one as far as the adequate explanation of this psychophysical connection is concerned. And exactly at this point conceivability arguments (either in their inverted or absent qualia version) will be introduced in order to prove that no such satisfactory replies are forthcoming or are in principle to be expected. For if we can easily and clearly conceive, as it is supposed by these arguments, that noxious or potentially noxious stimulation of the part or parts of our body is not followed by pain, but by some other sensation or no sensation at all, this will be taken as a reliable signal of the presence 
of the explanatory gap or the non-intelligibility of the relationship between such stimuli and pain.

But it is not so clear, as it might at first sight appear, that the demand for further intelligibility is really appropriate when the relationship between certain functional and phenomenal properties of pain is at stake. Likewise, it is not at all obvious that in the case of such relationships conceivability arguments actually have that epistemic force and import that Levine is attributing to them. As far as the connection between the functional and phenomenal properties of pain is concerned, the first thing to notice is that the pull towards the demand for further intelligibility or explanation of this connection is not, or should not be, as strong as it is in the case of the psychophysical correlation between the firing of certain neural structures and pain. The reason being that in the former case there is no conceptual heterogeneity or categorial difference between functional and phenomenal predicates that would make that demand seem immediately appropriate or quite understandable. On the contrary, functional properties are considered by us as properties that naturally go with phenomenal states which is proved by the fact that they are regularly referred to in the definitions of these states; that as a rule they feature in all our conceptions of such states. And it should be pointed out, that some relations between physical properties or magnitudes (for instance, the relationship between mass and distance captured by the gravitational constant) are taken for granted or considered not to be in need of further explanation, exactly because they fit smoothly into our theory of physical or form part of the same family, the same overall system of description.

However, it seems that the pull towards the demand for further intelligibility is not only less strong in the case of the association of the functional and phenomenal properties of pain, but that it is in principle directed more at the cases of real or possible disassociation of these properties than at their association. One might argue that in this sense the demand for further explanation or understanding of the relationship between noxious or potentially noxious stimuli and pain (or, to use more familiar terms, between injury and pain) would be inappropriate. Namely, as far as the intelligibility of this relationship 
is concerned, the proper question is not why injury elicits pain, but rather how it is possible for injury not to be followed by pain as well as for pain to appear without injury. What cries for explanation and where the requirement for adequate theory is most pressing are exactly the cases of the dissociation between pain and injury. To support this contention, it suffices to say that in pain research and theory out of seven most puzzling facts that are considered to require explanation by any new or adequate model of pain, four are exactly related to the dissociation or deviant connection between pain and injury:

... (1) the relationship between pain and injury is highly variable; (2) innocuous stimuli may produce pain; (3) the location of pain may be different from the location of damage; (4) pain may persist in the absence of injury or after healing. (Melzack and Wall, 1988, p. 165)

That injury, even a severe one, is not regularly followed by pain has been proved by clinical evidence: for instance, 37 percent of the patients who arrived at an emergency clinic with a variety of serious injuries "stated that they did not feel pain at the time of injury. The majority of these patients reported onset of pain within an hour of injury, although the delays were as long as 9 hours in some patients" (Melzack, Wall, and Ty, 1982, p. 33). On the other hand, there are cases of pain where no injury is involved: for example, it is reported that "as many as 60-78 percent of patients who suffer low back pain have no apparent physical signs" (Melzack and Wall, 1988, p. 58). "Gentle touch, vibration, and other non-noxious stimuli can trigger excruciating pain" (Melzack and Wall, 1988, p. 75) in causalgia and the neuralgias, and pain from hyperalgesic areas of the skin often arises after long delays and continues long after the removal of the stimulus. The clinical data also show that there is a mismatch between the site of injury and the site of pain exemplified in cases of referred or transferred pain to be found in appendicitis and angina pectoris. Besides, in many cases of chronic pain intense, debilitating pain persists for months or even years after all possible healing has been completed; and this pain, in contrast to acute pain, "is no longer the symptom of a disease but becomes a serious medical syndrome that requires attention for its own sake" (Melzack and Wall, 1988, p. 12). 
When presented with such cases of the dissociation or mismatch between pain and injury, we will immediately think of them as puzzling or mysterious; as something that demands explanation or calls for understanding. But that would not be the case, if the very relationship between pain and injury is considered by us to be puzzling or mysterious. For us, the mark of the intelligibility of that relationship is to be found in the fact that exactly the cases of the dissociation between pain and injury are considered as something that cries for explanation; as something that is deeply puzzling or mysterious. As we have already noted, in that sense the demand for further explanation of the relationship between pain and injury would be inappropriate. Generally speaking, the inappropriateness of these demands will critically depend on whether the pull towards such demands (the perplexity ratio) is directed more at the dissociation's than at the associations of the functional and phenomenal properties of the mental states under consideration.

For Levine and philosophers who share his interpretation of the explanatory gap problem, the conceivability of the dissociation between functional and phenomenal properties of a certain mental state is to be taken as a reliable mark of the non-intelligibility of their linkage. In the case that we are interested in, this would mean that we can pry apart in imagination pain and injury - conceive them as appearing one without the other - exactly because they seem to us to be arbitrarily stacked together; because the non-intelligibility of their relationship underlies or is the proper source of the apparent contingency of their connection. If that was not so, we could not so easily and clearly imagine injury without the feeling of pain, as well as pain without injury. We will not discuss the question whether we can really so easily and clearly imagine this state of affairs, but will rather concentrate on the intelligibility issues related to that which we are supposed to imagine: the absence of pain in the presence of severe injury. So let us imagine, for this purposes, that a needle has been severely pricked in the fingertip of our physical and functional doppelganger (conceived as much as possible in its full flesh and blood), or that he has pressed his fingertip against the hot electric light bulb, but that he feels no pain on that account. 
Assuming that we can clearly imagine this state of affairs, we will certainly be amazed by what we have imagined; we will immediately ask ourselves how it is possible and will regard the demand for further explanation as fully appropriate. If imagined cases of the dissociation between pain and injury will be regarded by us as something that is perplexing, puzzling or mysterious, the fact that we can conceive such state of affairs will not signal - contrary to Levine - the presence of an explanatory gap; it will not reveal the unintelligibility of the connection between pain and injury and thus will not show that the demand for further explanation of this connection is appropriate. That we can conceive injury without pain as well as pain without injury is rather to be explained by the simple fact that they are, to use traditional terminology, distinct existences. And as Hume has long ago remarked, whatever is distinct is separable in mind and imagination. But this doesn't mean at all that pain and injury are arbitrarily stacked together; that their relationship is mysterious or unintelligible because we do not see at all why they should be tightly connected. It only speaks against the identification of pain with injury and merely shows that pain cannot be a priori analyzed in terms of its causal role as it is attempted in the so-called perceptual view or model of pain. The general lesson to be learned from these considerations is that the a priori analysis of phenomenal concepts in functional terms is not a prerequisite for adequate or intelligible psychophysical explanations. Moreover, it has turned out that conceivability considerations are actually a poor guide or criterion for the assessment of the intelligibility or adequacy of such explanations.

One might complain that the experimental dull or burning pain artificially elicited by the electrical INMS of $\mathrm{C}$ fibers shows that the distinctive responsiveness of these fibers to the damaging or potentially damaging stimuli is not something that grounds (makes sensible or intelligible) their connection to the experience of pain. For in this case there is, indeed, a strong connection between the firing of the relevant kind of fibers and pain, although that activity was not induced by damaging or potentially damaging stimuli. But notice that in these cases there still exists the perfect or very close match between the projected field of the sensation (the site where the artificially induced 
pain is felt), and the receptive field of $\mathrm{C}$ fibers (the site or innervation territory where natural noxious stimuli instigate their activity). If that is the case, the role of damaging or potentially damaging stimulation as the bridging intelligible property has been preserved. Due to the role of $\mathrm{C}$ fibers in locognosia, instead of loosening the tie between pain and injury, the cases under consideration seem to tighten it in a quite unexpected way.

The intelligibility of the relationship between $\mathrm{C}$ fiber firing and pain (conferred or second-order intelligibility), established via the intelligibility of the functional-phenomenal relationship between injury and pain (conferring or first-order intelligibility), will provide an answer to the two "hard" problems of consciousness related to pain: namely, why $\mathrm{C}$ fiber firing evokes pain, and not some other kind of sensation? why it evokes any sensation at all? But an answer to the third "hard" problem of consciousness related to the physiological ground of the qualitative differences between various kinds of pain - why $\mathrm{C}$ fiber firing gives rise to dull, and not to sharp pain? - is to be sought in the different physical modes of activity of $\mathrm{C}$ fibers and other fibers responsible for pain. In other words, the physical (neurophysiological) explanation of sensory determinables (inter-modal differences) will have to rely on the functional properties common to certain phenomenal states and corresponding neural structures, while sensory determinates (intra-modal differences) will have a more direct neurophysiological account. When we are looking for such explanations of the qualitative differences between experiential determinates, we will have to search for property affinities between some features of the relevant neural structures and qualitative characteristics of the corresponding phenomenal states. For instance, the conspicuous difference in conduction velocities between A delta and C fibers, as well as the fact of the ongoing activity of $\mathrm{C}$ fibers after stimulation, could explain on property affinities ground why the former kind of fibers gives rise to sharp pain, while the latter kind of fibers gives rise dull pain. Within our general theoretical framework, the really hard problem will be to explain the functional properties that are characteristic for certain neural structures through the morphological properties of these structures. For instance: why $\mathrm{C}$ fibers and A delta fibers preferentially 
respond to noxious or potentially noxious stimuli, and not to some other kind of stimuli? Although we do not have nowadays answers to these question, and are thus with respect to them at the ignoramus stage, it seems that they are not of such a kind which has to lead us to the ignorabimus despair. 


\section{References}

Adrian, E.D. (1931), "The Messages in Sensory Nerve Fibres After Their Interpretation", Proceedings of the Royal Society B, 109, pp. $1-18$.

Barber, T.X. (1959), "Toward a Theory of Pain: Relief of Chronic Pain by Prefrontal Leucotomy, Opiates, Placebos, and Hypnosis", Psychological Bulletin, 56, pp. 430-460.

Bentham, J. (1948), An Introduction to the Principles of Morals and Legislation (New York: Hafner).

Berthier, M., Starkstein, S., and Leiguarda, R. (1988), "Pain Asymbolia: A Sensory-Limbic Disconnection Syndrome", Annals of Neurology, 24, pp. 41-49.

Biemond, A. (1956), "The Conduction of Pain Above the Level of the Thalamus Opticus", Archives of Neurology and Psychiatry, 75, pp. 221-231.

Brand, P. and Yancey, P. (1997), The Gift of Pain (Previously titled The Gift Nobody Wants) (Michigan: Zondervan Publishing House).

Burgess, P.R., and Perl, E.R. (1973), "Cutaneous Mechano-receptors and Nociceptors", in: A. Iggo (Ed.), Somato-sensory System, Handbook of Sensory Physiology, Vol. I (Berlin: Springer-Verlag), pp. 29-79.

Campbell, K. (1983), "Abstract Particulars and the Philosophy of Mind”, Australasian Journal of Philosophy, 61, pp. 129-141.

Chalmers, D. (1995), "Facing Up to the Problem of Consciousness", Journal of Consciousness Studies, 2, pp. 200-219.

Chapman, R.C., and Nakamura, Y. (1999), "A Passion of the Soul: An Introduction to Pain for Consciousness Researchers", Consciousness and Cognition, 8, pp. 391-422.

Chapman, R.C., Nakamura, Y., and Flores, L.Y. (2000), "How We Hurt: A Constructivist Framework for Understanding Individual Differences in Pain”, in: R.G. Kunzendorf and B. Wallace (Eds.), 
Individual Differences in Conscious Experience (Amsterdam: John Benjamin Publishing Co.), pp.17-44.

Chapman, W.P., Solomon, H.C., and Rose, A.S. (1950), "Measurements of Motor Withdrawal Reaction in Patients Following Frontal Lobotomy", in: M. Greenblat, R. Arnot and H.C. Solomon (Eds.), Studies in Lobotomy, New York: Grune \& Straton, pp. 386-392.

Churchland, Paul, and Churchland, Patricia (1981), "Functionalism, Qualia and Intentionality", Philosophical Topics, 12, pp. 121-145.

Cleeland, C.S., Nakamura, Y., Howland, E.W., Morgan, N.R., Edwards, K.R., and Backonja, M. (1996), "Effects of Oral Morphine on Cold Pressor Tolerance Time and Neuropsychological Performance", Neuropsychopharmacology, 15, pp. 252-262.

Coghill, R.C., Sang, C.N., Maisog, J.M., and Iadarola, M.A. (1999), "Pain Intensity Processing Within the Human Brain: A Bilateral, Distributed Mechanism", Journal of Neurophysiology, 82, pp. 1934-1943.

Cohen, R.A., Kaplan, R.F, Zuffante, P., Moser, D.J, Jenkins, M.A., Salloway, S., and Wilkinson, H. (1999), "Alteration of Intention and Self-Initiated Action Associated With Bilateral Anterior Cingulotomy", Journal of Neuropsychiatry and Clinical Neuroscience, 11, pp. 444-453.

Critchley, M. (1956), "Congenital Indifference to Pain", Annals of Internal Medicine, 45, pp. 737- 747.

Dearborn, G. (1932), "A Case of Congenital General Pure Analgesia", Journal of Nervous and Mental Diseases, 75, pp. 612-615.

Dennett, D.C. (1978), “Why You Can't Make a Computer That Feels Pain", Synthese, 38, pp. 415-456.

Denny-Brown, D. (1962), in: V.B. Mountcastle (Ed.), Intrahemispheric Relations and Cerebral Dominance (Baltimore), p. 244.

Descartes, R. (1968), The Philosophical Works of Descartes, vol. I, E.S. Haldane and G.R.T. Ross, editors and translators (Cambridge: Cambridge University Press). 
Dong, W.K., Chudler, E.H., Sugiyama, K., Roberts, V.J., and Hayashi, T. (1994), "Somatosensory, Multi-sensory, and Task-Related Neurons in Cortical Area 7b (PF) of Unanesthetized Monkeys", Journal of Neurophysiology; 72, pp. 542-564.

Dong, W.K., Hayashi, T., Roberts, V.J., Fusco, B.M., and Chudler, E.H. (1996), "Behavioral Outcome of Posterior Parietal Cortex Injury in the Monkey", Pain; 64, pp. 579-587.

Foltz, E.L, and White, L.E. (1962), "Pain 'Relief' by Frontal Cingulotomy”, Journal of Neurosurgery, 19, pp. 89-100.

Freeman, W., and Watts, J.W. (1950), Psychosurgery in the Treatment of Mental Disorders and Intractable Pain (Oxford: Blackwell, $2^{\text {nd }}$ ed.).

Geschwind, N. (1965), "Disconnexion Syndromes in Animals and Man”, Part I, Brain, 88, pp. 237-294.

Greenspan, J.D., Lee, R.R., and Lenz, F.A. (1999), "Pain Sensitivity as a Function of Lesion Location in the Parasylvian Cortex", Pain, 81, pp. 273-282.

Hall, K.R.L., and Stride, E. (1954), "The Varying Response to Pain in Psychiatry Disorders", British Journal of Medical Psychology, 27, pp. 48-60.

Halligan, P.W., Marshall, J.C., Hunt, M., and Wade, D.T. (1997), "Somatosensory Assessment: Can Seeing Produce Feeling?", Journal of Neurology, 244, pp. 190-203.

Hardcastle, V. (1999), The Myth of Pain (Cambridge, MA.: MIT Press).

Hardy, J.D., Wolff, H.G., and Goodel, H. (1952), Pain Sensations and Reactions (New York: Hafner).

Head, H. (1920), Studies in Neurology (London: Frowde, Hodder and Stoughton).

Hemphill, R.E., and Stengel, E. (1940), "A Study On Pure WordDeafness", Journal of Neurology and Psychiatry", 3, pp. 251-262. 
Hoogenraad, T.U., Ramos, L.M., and van Gijn, J. (1994), "Visually Induced Central Pain and Arm Withdrawal After Right Parietal Infarction", Journal of Neurology, Neurosurgery, and Psychiatry, 57, pp. $850-852$.

Hutchison, W.D., Davis, K.D., Lozano, A.M., Tasker, R.R., and Dostrovsky, J.O. (1999), "Pain-related Neurons in the Human Cingulate Cortex", Nature Neuroscience, 2, pp. 403-405.

International Association for the Study of Pain (IASP) Subcommittee on Classification, (1986), "Pain Terms: A Current List With Definitions and Notes on Usage", Pain, Supplement 3, (1986), pp. 216221.

Levine, J. (1983), "Materialism and Qualia: The Explanatory Gap Problem”, Pacific Philosophical Quarterly, 64, pp. 354-361.

Levine, J. (1991), “Cool Red”, Philosophical Psychology, 4, pp. 2740.

Lewis, D. (1966), "An Argument for the Identity Theory", The Journal of Philosophy, 63, pp. 17-25.

Lewis, D. (1995), "Should a Materialist Believe in Qualia?", Australasian Journal of Philosophy, 73, pp. 140-144.

Masson, C., Koskas, P., Cambier, J., and Masson, M. (1991), "Syndrome cortical pseudothalamique gauche et asymbolie à la douleur", Revue Neurologique, 10, pp. 668-670.

McMahon, S.B., and Koltzenburg, M. (1990), "Novel Classes of Nociceptors: Beyond Sherrington", Trends in Neurosciences, June, pp. 254-255.

McMurray, G.A. (1955), "Congenital Insensitivity to Pain and its Implications for Motivational Theory", Canadian Journal of Psychology, 9, pp. 121-131.

Melzack, R., and Wall, P. (1988), The Challenge of Pain (Harmondsworth: Penguin Books).

Melzack, R., Wall, P.D., and Ty, T.C. (1982), "Acute Pain in an Emergency Clinic: Latency of Onset and Descriptor Patterns Related to Different Injuries", Pain, 14, pp. 33-43. 
Mesulam, M.M., and Mufson, E. (1985), "The Insula of Reil in Man and Monkey. Architectonics, Connectivity and Function", In: Peters A. and Jones, E.G. (Eds.), Cerebral Cortex, Vol. 4 (New York: Plenum), pp. 179-226.

Millan, M.J. (1999), "The Induction of Pain: An Integrative Review", Progress in Neurobiology, 57, pp. 1-164.

Mitchell, S.W. (1872), Injuries of Nerves and Their Consequences (Philadelphia: J.B. Lippincott).

Nelkin, N. (1994), "Reconsidering Pain”, Philosophical Psychology, 7, pp. 325-343.

Ochoa, J., and Torebjörk, E. (1983), "Sensations Evoked by Intraneural Microstimulation of Single Mechanoreceptor Units Innervating the Human Hand", Journal of Physiology, 342, pp. 633665 .

Ochoa, J., and Torebjörk, E. (1989), "Sensations Evoked by Intraneural Microstimulation of $\mathrm{C}$ Nociceptor Fibers in Human Skin Nerves", The Journal of Physiology, 415, pp. 633-665.

Perl, E.R. (1984), "Characterization of Nociceptors and Their Activation of Neurons in the Superficial Dorsal Horn: First Step for the Sensation of Pain", in: L. Kruger and J.C. Liebeskind (Eds.), $A d-$ vances in Pain Research and Therapy, Vol. 6 (New York: Raven Press), pp. 23-51.

Pitcher, G. (1970), "Pain Perception”, The Philosophical Review, 79, pp. 368-393.

Ploner M., Freund, H.-J., and Schnitzler, A. (1999), "Pain Affect Without Pain Sensation in a Patient With a Postcentral Lesion", Pain, 81, pp. 211-214.

Pötzl, O., and Stengel, E. (1937), "Über das Syndrom Leitungsaphasie-Schmerzasymbolie”, Jahrbuch der Psychiatrie, 53, pp. 174-207.

Price, D.D., and Dubner, R. (1977), "Neurons That Subserve the Sensory-Discriminative Aspects of Pain", Pain, 3, pp. 307-338.

Price, D.D. (2000), "Psychological and Neural Mechanisms of the Affective Dimension of Pain", Science, 288, pp. 1769-1772. 
Ramachandran, V.S. (1998), "Consciousness and Body Image: Lessons from Phantom Limbs, Capgras Syndrome and Pain Asymbolia", Philosophical Transactions of the Royal Society of LondonSeries B: Biological Sciences, 353, pp. 1851-1859.

Robinson, C.J., and Burton, H. (1980), "Somatic Submodality Distribution Within the Second Somatosensory (SII), 7b, Retroinsula, Postauditory and Granular Insula of M. fascicularis", Journal of Comparative Neurology, 192, pp. 93-108.

Rubins, J.L., and Friedman, E.D. (1948), "Pain Asymbolia", Archives of Neurology and Psychiatry, 60, pp. 554-573.

Ryle, G. (1973), The Concept of Mind (Harmondsworth: Penguin).

Schilder, P., and Stengel, E. (1928), "Schmerzasymbolie", Zeitschrift für die gesamte Neurologie und Psychiatrie, 113, pp. 143-148.

Sherrington, C. (1900), "Cutaneous Sensations", in: E.A. Schäfer (Ed.), Textbook of Physiology, (London: Pentland), pp. 920-1001.

Sherrington, C. (1948), The Integrative Action of the Nervous System (originally published in 1906) (Cambridge: Cambridge University Press).

Sinclair, D.C (1967), Cutaneous Sensation (Oxford: Oxford University Press).

Talbot, J.D., Villemure, J.-G., Bushnell, M.C., and Duncan, G.H. (1995), "Evaluation of Pain Perception after Anterior Capsulotomy: A Case Report", Somatosensory and Motor Research, 12, pp. 115126.

Torebjörk, E., and Ochoa, J. (1980), "Specific Sensations Evoked by Activity in Single Identified Sensory Units in Man", Acta Physiologica Scandinavica, 110, pp. 445-447.

Torebjörk, E., Vallbo, A.B., and Ochoa, J. (1987), "Intraneural Microstimulation in Man", Brain, 110, pp. 1509-1529.

Trelles, J.O. (1978), “Asimbolia al dolor”, Revista Neuropsiquiatrica, 41, pp. 3-20.

Trigg, R. (1970), Pain and Emotion (Oxford: Clarendon Press). 
Tye, M. (1989), The Metaphysics of Mind (Cambridge: Cambridge University Press).

Tye, M. (1995), Ten Problems of Consciousness (Cambridge, Massachusetts: The MIT Press).

Vallbo, A.B., and Hagbarth, K.E. (1968), "Activity from Skin Mechanoreceptors Recorded Percutaneously in Awake Human Subjects", Experimental Neurology, 21, pp. 270-289.

Wade, J.B., Dougherty, L.M., Archer, C.R., and Price, D.D. (1996), "Assessing the Stages of Pain Processing: A Multivariate Analytical Approach", Pain, 68, pp. 157-167.

Wall, P.D., and McMahon, S.B. (1985), "Microneurography and Its Relation to Perceived Sensation: A Critical Review", Pain, 21, pp. 209-229.

Wall, P. (1999), Pain: The Science of Suffering (London: Weidenfeld $\&$ Nicolson).

Weinstein, E.A., Kahn, R.L., and Slote, W.H. (1955), "Withdrawal, Inattention, and Pain Asymbolia", Archives of Neurology and Psychiatry, 74, pp. 235-248.

Wittgenstein, L. (1968), Philosophical Investigations (Oxford: Basil Blackwell). 